\title{
Halide Anion Activated Reactions of Michael Acceptors with Tropylium Ion
}

Mohanad A. Hussein, Uyen P. N. Tran, Vien T. Huynh, Junming Ho, Mohan Bhadbhade, Herbert Mayr, Thanh Vinh Nguyen

Submitted date: 16/08/2019 - Posted date: 19/08/2019

Licence: CC BY-NC-ND 4.0

Citation information: Hussein, Mohanad A.; Tran, Uyen P. N.; Huynh, Vien T.; Ho, Junming; Bhadbhade, Mohan; Mayr, Herbert; et al. (2019): Halide Anion Activated Reactions of Michael Acceptors with Tropylium Ion. ChemRxiv. Preprint.

Tropylium bromide undergoes non-catalyzed, regioselective additions to a large variety of Michael acceptors. In this way, acrylic esters are converted into ß-bromo-a-cycloheptatrienyl-propionic esters. The reactions are interpreted by nucleophilic attack of bromide ions at the electron-deficient olefins and trapping of the incipient carbanion by the tropylium ion. Quantum chemical calculations were performed to elucidate the analogy to the amine or phosphine-catalyzed Rauhut-Currier reactions. Subsequent synthetic transformations of the bromo-cycloheptatrienylated adducts are reported.

File list (3)

Nguyen_TropBr_ChemRxiv.pdf (1.18 MiB)

view on ChemRxiv • download file

TOC_Nguyen.jpeg (79.72 KiB)

view on ChemRxiv • download file

Nguyen_TropBr_SI_ChemRxiv.pdf (5.33 MiB)

view on ChemRxiv • download file 


\title{
Halide Anion Activated Reactions of Michael Acceptors with Tropylium Ion
}

\author{
Mohanad A. Hussein, ${ }^{[\mathrm{a}]}$ Uyen P. N. Tran, ${ }^{[\mathrm{a}]}$ Vien T. Huynh,,${ }^{[\mathrm{b}]}$ Junming Ho, ${ }^{[\mathrm{a}]}$ Mohan Bhadbhade,${ }^{[\mathrm{c}]}$ \\ Herbert Mayr ${ }^{[\mathrm{d}]}$ and Thanh V. Nguyen ${ }^{*[a]}$
}

\begin{abstract}
Tropylium bromide undergoes non-catalyzed, regioselective additions to a large variety of Michael acceptors. In this way, acrylic esters are converted into ß-bromo- $\alpha-$ cycloheptatrienyl-propionic esters. The reactions are interpreted by nucleophilic attack of bromide ions at the electron-deficient olefins and trapping of the incipient carbanion by the tropylium ion. Quantum chemical calculations were performed to elucidate the analogy to the amine or phosphine-catalyzed Rauhut-Currier reactions. Subsequent synthetic transformations of the bromocycloheptatrienylated adducts are reported.
\end{abstract}

Michael acceptors are versatile building blocks in organic synthesis. ${ }^{[1]}$ Their activation by tertiary amines and phosphines in Morita-Baylis-Hillman and Rauhut-Currier reactions is wellestablished (Scheme 1)..$^{[1,2]}$ In recent years $N$-heterocyclic carbene-catalyzed reactions of Michael acceptors have become increasingly popular. ${ }^{[1 b-d, 3][4]}$ To our knowledge, halide ions have so far not been used to initiate reactions of Michael acceptors because the Lewis basicity of $\mathrm{Br}^{-}$and $\mathrm{Cl}^{-}$towards $\mathrm{C}$-centered Lewis acids is $4-10$ orders of magnitude smaller than that of the commonly employed phosphines and amines. ${ }^{[5]}$ In this communication we will demonstrate, however, that halide ion addition to Michael acceptors is possible when the emerging carbanion is stabilized through ion pairing with a tropylium ion, ${ }^{[6]}$ which possibly represents a first example of a more general reaction principle for linking Michael acceptors with stabilized carbocations. The transformations reported in this communication provide an efficient access to a wide range of synthetically valuable ${ }^{[7]}$ substituted cycloheptatrienes.

While tropylium salts have been known to trigger polymerization reactions of electron-rich alkenes, ${ }^{[8]}$ we found that tropylium bromide (2a) reacted with electron-deficient alkenes such as methyl acrylate (1a) to give the bromocycloheptatrienylated adduct $4 \mathrm{a}$ in a highly regioselective manner (Procedure $\mathrm{A}$, Table 1). The resulting products correspond to those of the $\mathrm{TiCl}_{4}$-induced Baylis-Hillman reactions ${ }^{[9]}$ and the tandem thiolmediated Rauhut Currier reactions. ${ }^{[10]}$ One may consider these reactions as the nucleophilic counterparts of the Lewis acid catalyzed additions of alkyl halides to nucleophilic alkenes (Prins reaction $)^{[11]}$ and as heterolytic variants of the Kharasch radical additions ${ }^{[12]}$ to olefins. This new chemical transformation thus

[a] M. A. Hussein, Dr. U. P. N. Tran, Dr. J. Ho and Dr. T. V. Nguyen School of Chemistry, UNSW Sydney

E-mail: t.v.nguyen@unsw.edu.au

[b] Dr. V. T. Huynh, School of Chemistry, University of Sydney

[c] Dr M. Bladblade, Mark Wainwright Analytical Centre, UNSW Sydney

[d] Prof. Dr. H. Mayr, Department Chemie, LMU München

Supporting information for this article is given via a link at the end of the document.

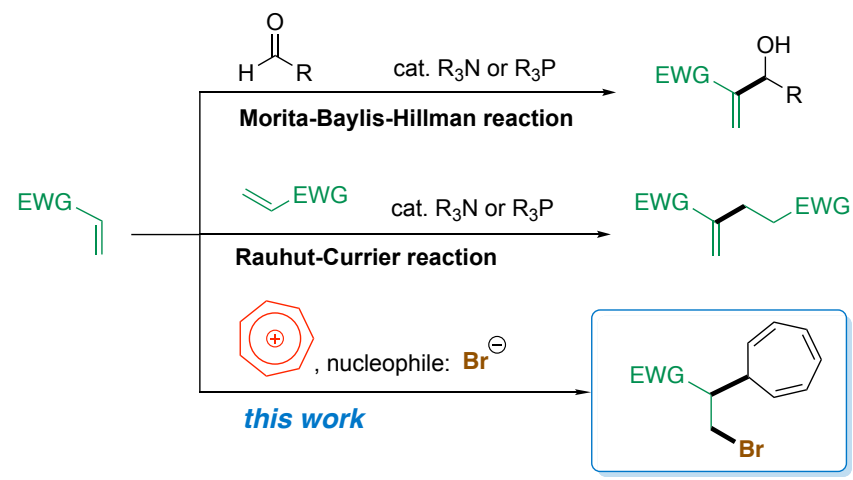

Scheme 1. Nucleophilic activation of Michael acceptors for reactions with electrophiles.

allows direct haloalkylation of electron-deficient olefins in a catalyst-free, metal-free and non-radical fashion. ${ }^{[12 b, 13]}$

Optimization of the reaction with methyl acrylate $(\mathbf{1} \mathbf{a}$, see page $\mathrm{S} 3$ in the $\mathrm{SI})^{[14]}$ showed that slightly better yields of $4 \mathrm{a}$ were obtained when we used a combination of tropylium tetrafluoroborate (2b) and TMSBr in DCE (Procedure B, Table 1), which is a practical way to avoid handling of the hygroscopic tropylium bromide. ${ }^{[15]}$ Tropylium salt $\mathbf{2} \mathbf{b}$ reacted with $\mathrm{Me}_{3} \mathrm{SiBr}$ (TMSBr) instantaneously to give TMSF and a mixture of tropylium bromide and $\mathrm{BF}_{3}$ (which coordinate to give tropylium bromotrifluoroborate ${ }^{[16]}$ see page S5 in the SI for more details). This mixture renders the bromocycloheptatrienylation process in a similar way as tropylium bromide. Other bromide sources such as alkali- or tetrabutylammonium bromide in combination with tropylium tetrafluoborate gave unsatisfactory results. ${ }^{[14]}$

Under these optimized reaction conditions, we were able to carry out the bromocycloheptatrienylations of a series of monosubstituted Michael acceptors (Table 1) with electrophilicity parameters ${ }^{[17]}$ ranging from $-24<E<-14$ in good yields. Some substrates were functionalized using both Procedures $A$ and $B$ (4a, 4b, 4c, 4d, 4i, 4j, 4I, 4n, Table 1). Acrylates with bulky alkyl groups (1e and $\mathbf{1 g}$ ), reacted with cleavage of the esters to form the corresponding carboxylic acid 4e. C-C multiple bonds without acceptor substituents (1i and $\mathbf{1 j}$ ) and amide groups (1k $)$ tolerated the reaction conditions. However, vinyl sulfone and several 1,1- and 1,2-disubstituted Michael acceptors did not react under various conditions (see page S16 in the SI for more details on other non-reacting substrates). ${ }^{[14]}$ Replacement of $\mathrm{TMSBr}$ by either TMSCl or TMSI in Procedure B (Table 1) afforded comparable yields of the chloro- and iodocycloheptatrienylated adducts $\mathbf{4 a C l}$ and $4 \mathbf{a l}$, respectively. Tropylium fluoride did not react, probably because of the covalent nature of this compound. 
Table 1. Halocycloheptatrienylation reactions of Michael acceptors. ${ }^{[a]}$

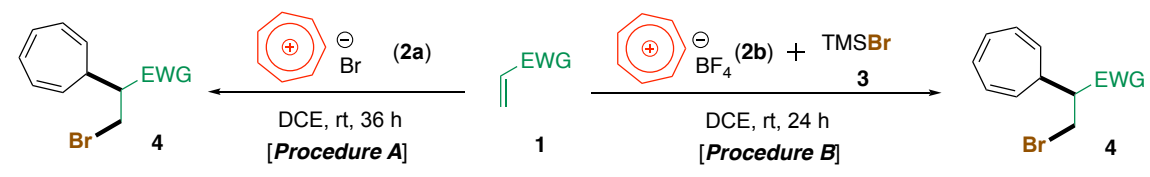

\begin{tabular}{|c|c|c|c|c|c|c|c|c|}
\hline Substrate $e^{[b]}$ & Product & Yield & Substrate $^{[\mathrm{b}]}$ & Product & Yield & Substrate $^{[\mathrm{b}]}$ & Product & Yield \\
\hline $\begin{array}{c}\mathrm{O}_{2} \mathrm{Me} \\
\mathbf{1 a}\end{array}$ & & $\begin{array}{l}67 \%[\boldsymbol{A}] \\
75 \%[\boldsymbol{B}]\end{array}$ & & & $\begin{array}{l}73 \% \\
{[B]}\end{array}$ & & & $91 \%[B]$ \\
\hline $\begin{array}{c}\mathrm{CO}_{2} \mathrm{Me} \\
\mathbf{1 a}\end{array}$ & & $\begin{array}{l}55 \% \\
\text { [B] with } \\
\text { TMSCl }\end{array}$ & & & $\%[B]$ & & & $\begin{array}{l}77 \%[\boldsymbol{A}] \\
79 \%[B]\end{array}$ \\
\hline$\| \begin{array}{c}\mathrm{CO}_{2} \mathrm{Me} \\
\mathbf{1 a}\end{array}$ & & $\begin{array}{l}66 \% \\
{[B] \text { with }} \\
\text { TMSI } \\
\text { as } 3\end{array}$ & & & $\%[B]$ & $1 \mathrm{~m}$ & & $71 \%[B]$ \\
\hline $\begin{array}{r}\mathrm{CO}_{2} \mathrm{Et} \\
\mathbf{1 b}\end{array}$ & $4 b$ & $\begin{array}{l}70 \%[\boldsymbol{A}] \\
73 \%[\boldsymbol{B}]\end{array}$ & $\begin{array}{c}\text { Acrylate } \\
\text {-PEG5- } \\
\text { Acrylate } \\
\mathbf{1 h}^{[\mathrm{cl}]}\end{array}$ & & $\begin{array}{l}63 \% \\
{[B]}\end{array}$ & & & $\begin{array}{l}68 \%[\boldsymbol{A}] \\
76 \%[B]\end{array}$ \\
\hline$\|^{\mathrm{CO}_{2}{ }^{n} \mathrm{Bu}} \begin{array}{c}\mathbf{1 c} \\
\end{array}$ & & $\begin{array}{l}59 \%[\boldsymbol{A}] \\
68 \%[\boldsymbol{B}]\end{array}$ & & & $\begin{array}{l}\%[\boldsymbol{A}] \\
\%[\boldsymbol{B}]\end{array}$ & 10 & & $7 \%[\boldsymbol{B}]^{[c]}$ \\
\hline $\begin{array}{c}\mathrm{CO}_{2}{ }^{i} \mathrm{Bu} \\
\mathbf{1 d}\end{array}$ & & $\begin{array}{l}64 \%[A] \\
71 \%[B]\end{array}$ & & & $\begin{array}{l}\%[A] \\
\%[B]\end{array}$ & & & $64 \%[B]$ \\
\hline
\end{tabular}

[a] Reaction conditions: [A] 1 (1.0 mmol) and 2a (1.0 mmol) in DCE (10 mL), rt, $36 \mathrm{~h}$; [B] 1 (1.0 mmol) and $\mathbf{2 b}(1.0 \mathrm{mmol})$ in DCE (10 mL) then $\mathbf{3}(1 \mathrm{mmol})$, rt, $24 \mathrm{~h}$. [b] When there is steric hindrance at the $\alpha$-position of the electron-withdrawing group, the reaction did not work. A full list of non-reactive Michael acceptors we tested can be found page S16 in the SI. [c] With 2.0 equivalents of $\mathbf{2 a}$ or $\mathbf{2} \mathbf{b}$ and $\mathbf{3}$, the mono-cycloheptatrienylated product was only formed in trace amounts.

Butenolide 1q reacted differently and gave cycloheptatriene $\mathbf{4 q}$ and $\gamma$-ketoacid $\mathbf{4 q}$ '. Treatment of the crude mixture of $\mathbf{4 q}$ and 4q' with aqueous $\mathrm{HCl}$ solution resulted in the quantitative formation of $\mathbf{4 q}$ ' (Scheme 2). This reaction can be rationalized by the tautomerization of butenolide 1q to 2-hydroxyfuran, which is subsequently attacked by the tropylium ion to produce $\mathbf{4 q}$ (see pages $\mathrm{S} 17-18$ in the SI for a possible mechanistic pathway for the formation of $\left.\mathbf{4} \mathbf{q}^{\prime}\right)$.

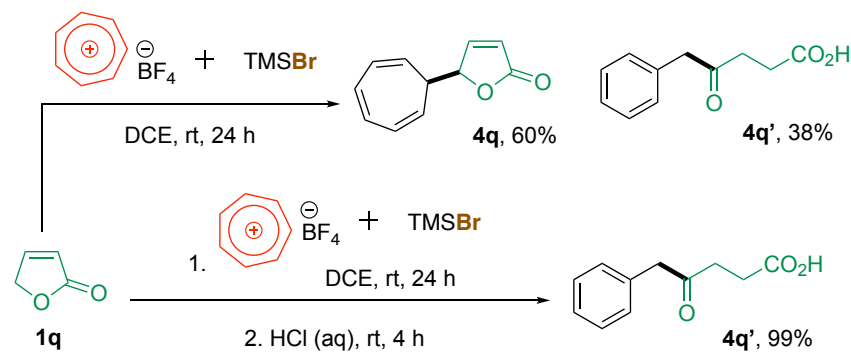

Electron-deficient alkynes behaved similarly to their olefinic analogues. Thus propiolates $\mathbf{5 a}, \mathbf{b}$ and dimethyl acetylene dicarboxylate 5c (Scheme 3) reacted smoothly with tropylium tetrafluoroborate and $\mathrm{TMSBr}$ to give E/Z-mixtures of the corresponding bromoalkenes $6 \mathbf{6 a - c}$, with the $Z$-isomers being major products.

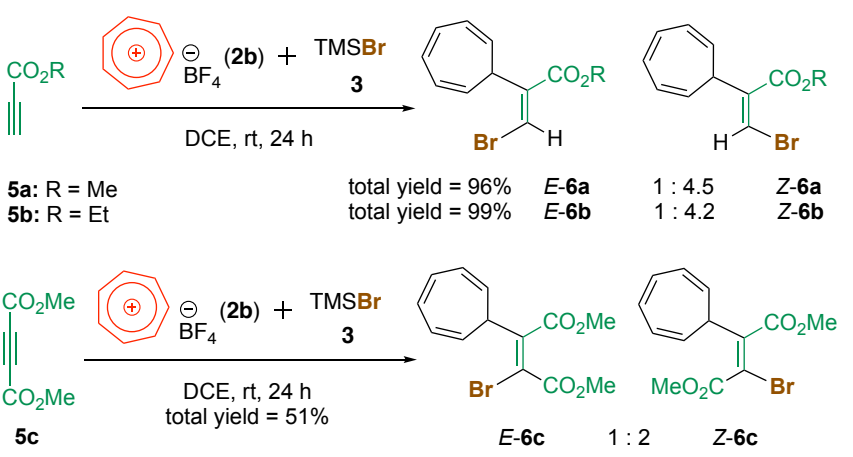

Scheme 3. Halocycloheptatrienylation reactions of electron-deficient alkynes. 


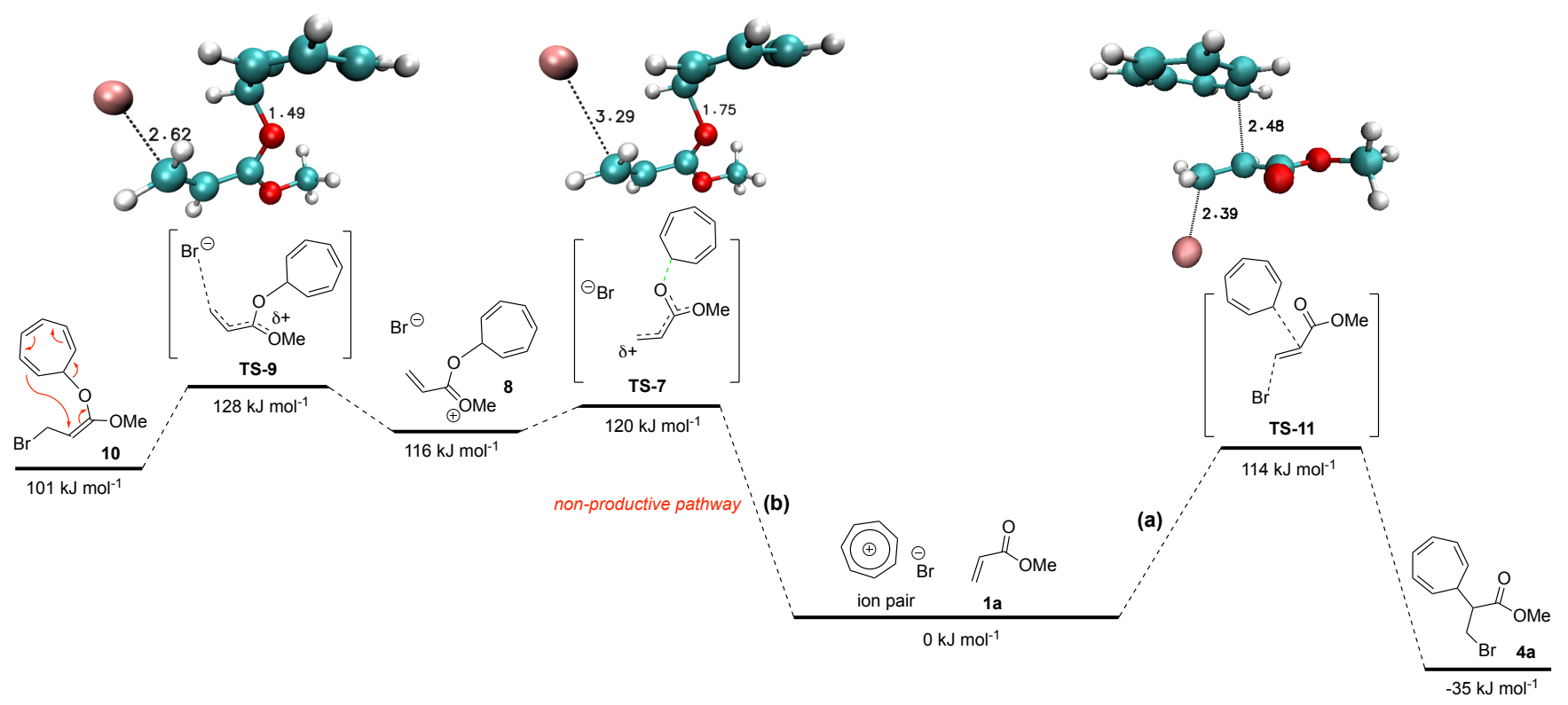

Figure 1. M06-2X/6-31+G(d,p) + SMD (dichloroethane) free energy profile. Distances are shown in $\AA$. Energy levels are not to scale.

Density functional theory $\left(\mathrm{M} 06-2 \mathrm{X} / 6-31+\mathrm{G}(\mathrm{d}, \mathrm{p})^{[18]}\right.$ and $\mathrm{SMD}^{[19]}$ ) calculations were carried out to investigate the mechanism of the reaction of methyl acrylate (1a) with tropylium bromide 2a, which exists as ion-pair in DCE solution, $21.8 \mathrm{~kJ}$ $\mathrm{mol}^{-1}$ more stable than the separate ions. ${ }^{[20]}$ In contrast to the mechanism of related Baylis-Hillman and Rauhut-Currier reactions, where DABCO was calculated to add to methyl acrylate (1a) with endergonic formation of a zwitterion $\left(\Delta G^{0}=\right.$ $+65 \mathrm{~kJ} \mathrm{~mol}^{-1}$ in acetonitrile $)^{[21]}$ the analogous covalent adduct of $\mathrm{Br}^{-}$to $1 \mathrm{a}$ was neither found as a local minimum in the gas phase nor in solution. This finding is not surprising in view of the fact that the DABCO-adduct is in a very shallow local minimum (barrier for decomposition $8 \mathrm{~kJ} \mathrm{~mol}^{-1}$ ) [21] $^{2}$ and the Lewis basicity of $\mathrm{Br}^{-}$toward C-centers is $36 \mathrm{~kJ} \mathrm{~mol}^{-1}$ lower than that of DABCO. ${ }^{[5]}$

However, when tropylium bromide (2a) instead of free $\mathrm{Br}^{-}$ was combined with methyl acrylate 1a in DCE, the nucleophilic attack of $\mathrm{Br}^{-}$at $1 \mathrm{a}$ was electrophilically assisted by the tropylium ion as shown by TS-11 in Figure 1 (pathway a). Though adduct 4a is thus formed by a concerted pathway, the Wiberg bond indices $(\mathrm{C}-\mathrm{Br}=0.502, \mathrm{C}-\mathrm{C}=0.187)$ show that the formation of the $\mathrm{C}-\mathrm{Br}$ bond is considerably further advanced in TS-11 than the formation of the new $\mathrm{C}-\mathrm{C}$ bond. Foregoing nucleophilic attack by bromide supported by electrophilic attack of the tropylium ion can also be derived from the relative lengths of the two new $\sigma$-bonds in the transition state. Whereas the new $\mathrm{C}-\mathrm{Br}$ bond $(2.39 \AA)$ has the 1.23-fold length of a covalent $\mathrm{C}-\mathrm{Br}$ bond, the new $\mathrm{C}-\mathrm{C}$ bond $(2.48 \AA)$ has the 1.63-fold length of the C-C bond in the product.

An alternative pathway for the reaction of tropylium bromide with 1a may be initiated by electrophilic attack of the tropylium ion at the carbonyl oxygen of $\mathbf{1 a}$ to give carboxonium ion $\mathbf{8}$ (Figure 1, pathway b). This intermediate may either undergo a retroaddition with regeneration of the reactants or combine with $\mathrm{Br}^{-}$to give ketene acetal 10, which might undergo a subsequent sigmatropic rearrangement (red arrows in structure 10) to yield 4a. Though we were not able to identify a transition state for the last step, pathway (b) can already be ruled out due to the fact that the barrier for formation of $\mathbf{1 0}$ is higher than that for the direct formation of $4 a$ via pathway (a).

An anti-addition as shown in TS-11 may also be preferred in reactions with acetylenic Michael acceptors. This could account for the predominant formation of the Z-isomers of 6a-c (Scheme 3).

Cycloheptatriene derivatives are useful building blocks in organic synthesis, as demonstrated by the Echavarren group and others. ${ }^{[\mathrm{aa}, 7 \mathrm{~b}]}$ To demonstrate the synthetic utility of our readily accessible bromocycloheptatriene products, some preliminary work on further functionalization of a representative compound, product $\mathbf{4 a}$, was carried out and reported in Scheme 4. Dehydrobromination reaction of product $4 \mathbf{a}$ afforded $95 \%$ of cycloheptatrienyl acrylate 12 , potentially a useful monomer for redox active polymers. Compound 12 can be considered as a formal Morita-Baylis-Hillman-type product from methylacrylate 1a and tropylium ion as the electrophile. However, such products cannot be formed directly by phosphine $\left(\mathrm{Ph}_{3} \mathrm{P}\right)$ or tertiary amine (DABCO) mediated reactions, as the tropylium ion will coordinate to these nucleophilic catalysts and thus deactivate them.

On the other hand, because cycloheptatriene is a versatile moiety for cycloaddition reaction, ${ }^{[22]}$ we thus demonstrated this reactivity by a reaction between $4 \mathbf{a}$ and maleimide, which resulted in the norcaradiene-type [4+2] cycloaddition adduct $\mathbf{1 3}$ as a mixture of two enantiomers. The structure of this compound was confirmed by X-ray crystallographic analysis (see X-ray crystal structure in page $\mathrm{S} 30$ in the $\mathrm{SI}),{ }^{[23]}$ which revealed the exclusive endo-selectivity and the diastereoselectivity of the cycloaddition reaction. 


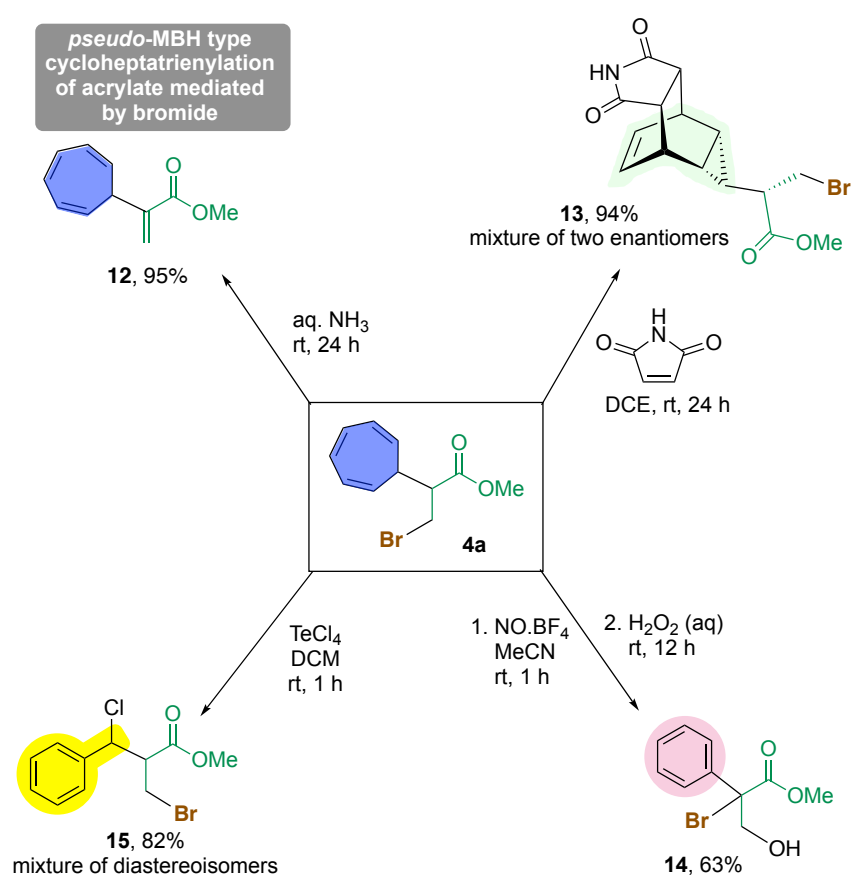

Scheme 4. Further functionalizations of bromocycloheptatrienylated product.

Given that tropylium moiety (C7) can potentially be oxidative ring-contracted to give the phenyl ring $(\mathrm{C} 6),{ }^{[2]}$ we treated cycloheptatriene $4 \mathrm{a}$ with nitrosonium tetrafluoroborate $\mathrm{e}^{[25]}$ to generate the tropylium derivative in situ then directly oxidize it in the same reaction pot with hydrogen peroxide. ${ }^{[26]}$ Interestingly, the reaction worked as expected to give the ring-contracted product 14 in good yield. In another study, treatment of adduct $4 \mathbf{a}$ with tellurium tetrachloride ${ }^{[27]}$ led to the rearrangement of the cycloheptatriene ring to the benzyl group in conjunction with the addition of one chlorine atom to afford product 15, an interesting dihalogenated ester. All products 12-15 in Scheme 4 are novel compounds and accessible in 1-2 steps from methylacrylate 1a using our newly developed chemical transformation.

In conclusion, we have developed a new method using. halide ions to activate electron-deficient olefins for nucleophilic attack at tropylium ions. Since one can expect analogous reactions of Michael acceptors with other types of stabilized carbocations (including iminium ions), the generality of this approach for the difunctionalization of electron-deficient olefins in a catalyst-free, metal-free and non-radical fashion is presently investigated. The adducts produced by our protocol have enormous potential in organic synthesis as the cycloheptatriene moiety can be used as a building block to quickly access unprecedented structures.

\section{Acknowledgements}

The authors thank the Australian Research Council (grant FT180100260 to TVN and DE160100807 to JH) for financial support. MAH thanks the Iraqi HCED for sponsoring his Ph.D. scholarship. Preliminary experiment by $\mathrm{Mr}$ Klaus Omoregbee is acknowledged. The authors thank A/Prof Jason Harper (UNSW) for helpful discussion. JH acknowledges support from the $\mathrm{NCl}$ and Intersect NSW for supercomputer resources.

Keywords: Michael acceptors $\bullet$ bromide $\bullet$ tropylium

[1] (a) D. Seebach, Angew. Chem. Int. Ed. 1990, 29, 1320-1367; (b) X. Bugaut, F. Glorius, Chem. Soc. Rev. 2012, 41, 3511-3522; (c) X.-Y. Chen, S. Ye, Org. Biomol. Chem. 2013, 11, 7991-7998; (d) D. M. Flanigan, F. Romanov-Michailidis, N. A. White, T. Rovis, Chem. Rev. 2015, 115, 9307-9387; (e) H. Guo, Y. C. Fan, Z. Sun, Y. Wu, O. Kwon, Chem. Rev. 2018, 118, 10049-10293.

[2] (a) C. E. Aroyan, A. Dermenci, S. J. Miller, Tetrahedron 2009, 65, 40694084; (b) D. Basavaiah, B. S. Reddy, S. S. Badsara, Chem. Rev. 2010, 110, 5447-5674; (c) Y. Wei, M. Shi, Chem. Rev. 2013, 113, 6659-6690; (d) H. Ni, W.-L. Chan, Y. Lu, Chem. Rev. 2018, 118, 9344-9411.

[3] (a) M. N. Hopkinson, C. Richter, M. Schedler, F. Glorius, Nature 2014, 510, 485; (b) C. Fischer, S. W. Smith, D. A. Powell, G. C. Fu, J. Am. Chem. Soc. 2006, 128, 1472-1473; (c) A. T. Biju, M. Padmanaban, N. E. Wurz, F. Glorius, Angew. Chem. Int. Ed. 2011, 50, 8412-8415; (d) S.-i. Matsuoka, Y. Ota, A. Washio, A. Katada, K. Ichioka, K. Takagi, M. Suzuki, Org. Lett. 2011, 13, 3722-3725; (e) T. Kato, Y. Ota, S.-i. Matsuoka, K. Takagi, M. Suzuki, J. Org. Chem. 2013, 78, 8739-8747; (f) S.-i. Matsuoka, S. Namera, A. Washio, K. Takagi, M. Suzuki, Org. Lett. 2013, 15, 5916-5919; (g) T. Kato, S.-i. Matsuoka, M. Suzuki, J. Org. Chem. 2014, 79, 4484-4491.

[4] (a) S. E. Denmark, G. L. Beutner, Angew. Chem. Int. Ed. 2008, 47, 1560-1638; (b) E. Vedejs, S. E. Denmark, Lewis Base Catalysis in Organic Synthesis, 3 Volume Set, Wiley, 2016.

[5] H. Mayr, J. Ammer, M. Baidya, B. Maji, T. A. Nigst, A. R. Ofial, T. Singer, J. Am. Chem. Soc. 2015, 137, 2580-2599.

[6] (a) G. Merling, Ber. Dtsch. Chem. Ges 1891, 24, 3108-3126; (b) W. Von E.Doering, L. H. Knox, J. Am. Chem. Soc. 1954, 76, 3203-3206; (c) T. V. Nguyen, A. Bekensir, Org. Lett. 2014, 16, 1720-1723; (d) T. V. Nguyen, M. Hall, Tetrahedron Lett. 2014, 55, 6895-6898; (e) T. V. Nguyen, D. J. M. Lyons, Chem. Commun. 2015, 51, 3131-3134; (f) D. J. M. Lyons, R. D. Crocker, M. Blümel, T. V. Nguyen, Angew. Chem. Int. Ed. 2017, 56, 1466-1484; (g) D. J. M. Lyons, R. D. Crocker, D. Enders, T. V. Nguyen, Green Chem 2017, 3993-3996; (h) M. A Hussein, V T. Huynh, R. Hommelsheim, R. M. Koenigs, T. V. Nguyen, Chem. Commun. 2018, 54, 12970-12973; (i) D. Lyons, R. Crocker, T. V. Nguyen, Chem. Eur. J. 2018, 24, 10959-10965; (j) G. Oss, S. D. de Vos, K. N. H. Luc, J. B. Harper, T. V. Nguyen, J. Org. Chem. 2018, 83, 1000-1010; (k) G. Oss, J. Ho, T. V. Nguyen, Eur. J. Org. Chem. 2018, 3974-3981; (I) U. P. N. Tran, G. Oss, D. P. Pace, J. Ho, T. V. Nguyen, Chem. Sci. 2018, 9, 5145-5151.

[7] (a) M. Mato, C. García-Morales, A. M. Echavarren, ChemCatChem 2018, 0; (b) C. R. Solorio-Alvarado, Y. Wang, A. M. Echavarren, J. Am. Chem. Soc. 2011, 133, 11952-11955; (c) P. R. McGonigal, C. de León, Y. Wang, A. Homs, C. R. Solorio-Alvarado, A. M. Echavarren, Angew. Chem. Int. Ed. 2012, 51, 13093-13096; (d) Y. Wang, P. R. McGonigal, B. Herlé, M. Besora, A. M. Echavarren, J. Am. Chem. Soc. 2014, 136, 801809; (e) Y. Wang, M. E. Muratore, Z. Rong, A. M. Echavarren, Angew. Chem. Int. Ed. 2014, 53, 14022-14026; (f) S. Ferrer, A. M. Echavarren, Angew. Chem. Int. Ed. 2016, 55, 11178-11182; (g) B. Herlé, P. M. Holstein, A. M. Echavarren, ACS Catal. 2017, 7, 3668-3675; (h) M. Vayer, R. Guillot, C. Bour, V. Gandon, Chem. Eur. J. 2017, 23, 1390113905; (i) X. Yin, M. Mato, A. M. Echavarren, Angew. Chem. Int. Ed. 2017, 56, 14591-14595; (j) A. N. Bismillah, J. Sturala, B. M. Chapin, D. S. Yufit, P. Hodgkinson, P. R. McGonigal, Chem. Sci. 2018, 9; (k) M. Mato, B. Herle, A. M. Echavarren, Org. Lett. 2018, 20, 4341-4345; (I) M. Vayer, R. Guillot, C. Bour, V. Gandon, J. Org. Chem. 2018, 83, 11309-11317; (m) A. M. Echavarren, M. Mato, Angew. Chem. Int. Ed. 2019, 57.

[8] A. Ledwith, in Addition and Condensation Polymerization Processes, Vol. 91, American Chemical Society, 1969, pp. 317-334..

[9] (a) G. Li, J. Gao, H.-X. Wei, M. Enright, Org. Lett. 2000, 2, 617-620; (b) Z. Han, S. Uehira, H. Shinokubo, K. Oshima, J. Org. Chem. 2001, 66, 7854-7857; (c) T. Iwamura, M. Fujita, T. Kawakita, S. Kinoshita, S.-i. Watanabe, T. Kataoka, Tetrahedron 2001, 57, 8455-8462; (d) M. Baidya, G. Y. Remennikov, P. Mayer, H. Mayr, Chem. Eur. J. 2010, 16, 13651371; (e) C. Patel, R. B. Sunoj, J. Org. Chem. 2010, 75, 359-367; (f) M. Shi, J.-K. Jiang, Y.-S. Feng, Org. Lett. 2000, 2, 2397-2400.

[10] (a) P. M. Brown, N. Käppel, P. J. Murphy, Tetrahedron Lett. 2002, 43, 8707-8710; (b) P. M. Brown, N. Käppel, P. J. Murphy, S. J. Coles, M. B. Hursthouse, Tetrahedron 2007, 63, 1100-1106; (c) C. E. Aroyan, S. J. Miller, J. Am. Chem. Soc. 2007, 129, 256-257.

[11] (a) J. Boseken, H. J. Prins, Versl. Akad. Wetenschappen (Amsterdam) 1910, 19, 776; (b) L. Schmerling, J. Am. Chem. Soc. 1945, 67, 11521154; (c) H. Mayr, W. Striepe, J. Org. Chem. 1983, 48, 1159-1165.

[12] (a) M. S. Kharasch, E. V. Jensen, W. H. Urry, Science 1945, 102, $128-$ 128; (b) X.-W. Lan, N.-X. Wang, Y. Xing, Eur. J. Org. Chem. 2017, 2017, 5821-5851. 
[13] (a) X. Wu, W. Hao, K.-Y. Ye, B. Jiang, G. Pombar, Z. Song, S. Lin, J. Am Chem. Soc. 2018, 140, 14836-14843; (b) N. Fu, Y. Shen, A. R. Allen, L. Song, A. Ozaki, S. Lin, ACS Catal. 2019, 9, 746-754.

[14] See the Supporting Information for more details.

[15] (a) R. Caputo, C. Ferreri, G. Palumbo, E. Wenkert, Tetrahedron Lett. 1984, 25, 577-578; (b) A. R. Bassindale, T. Stout, Tetrahedron Lett. 1984, 25, 1631-1632.

[16] J. S. Hartman, G. J. Schrobilgen, Inorganic Chemistry 1972, 11, 940-951.

[17] D. S. Allgäuer, H. Jangra, H. Asahara, Z. Li, Q. Chen, H. Zipse, A. R. Ofial, H. Mayr, J. Am. Chem. Soc. 2017, 139, 13318-13329.

[18] Y. Zhao, D. G. Truhlar, Theor. Chem. Acc. 2008, 120, 215-241.

[19] A. V. Marenich, C. J. Cramer, D. G. Truhlar, J. Phys. Chem. B 2009, 113, 6378-6396

[20] The computational calculations for mechanistic details of Procedure B are rather complicated and will be reported in a follow-up study in due course.

[21] Y. Li, S. Du, Int. J. Quantum Chem. 2017, 117, e25325.
[22] (a) W. D. Mackay, J. S. Johnson, Org. Lett. 2016, 18, 536-539; (b) K. N. Houk, R. B. Woodward, J. Am. Chem. Soc. 1970, 92, 4143-4145; (c) R. Yokoyama, S. Ito, M. Watanabe, N. Harada, C. Kabuto, N. Morita, J. Chem. Soc., Perkin Trans. 2001, 2257-2261; (d) A. Tenaglia, S. Gaillard, Angew. Chem. Int. Ed. 2008, 47, 2454-2457; (e) G. Hilt, A. Paul, C. Hengst, Synthesis 2009, 2009, 3305-3310; (f) H. Clavier, K. L. Jeune, I. d. Riggi, A. Tenaglia, G. Buono, Org. Lett. 2011, 13, 308-311.

[23] Atomic coordinates, bond lengths and angles and displacement parameters for compound 11 have been deposited at the Cambridge Crystallographic Data Centre (CCDC No. 1922409). This data can be obtained free-of-charge via http://www.ccdc.cam.ac.uk/data request/cif, by emailing data_request@ccdc.cam.ac.uk.

[24] A. Tamoto, N. Aratani, H. Yamada, Chem. Eur. J. 2017, 23, 1638816392

[25] G.-W. Zhang, Q. Shi, C.-F. Chen, Chem. Commun. 2017, 53, 2582-2585.

[26] M. E. Volpin, D. N. Kursanov, V. G. Dulova, Tetrahedron 1960, 8, 33-37.

[27] M. Albeck, T. Tamari, M. Sprecher, J. Org. Chem. 1983, 48, 2276-2278. 



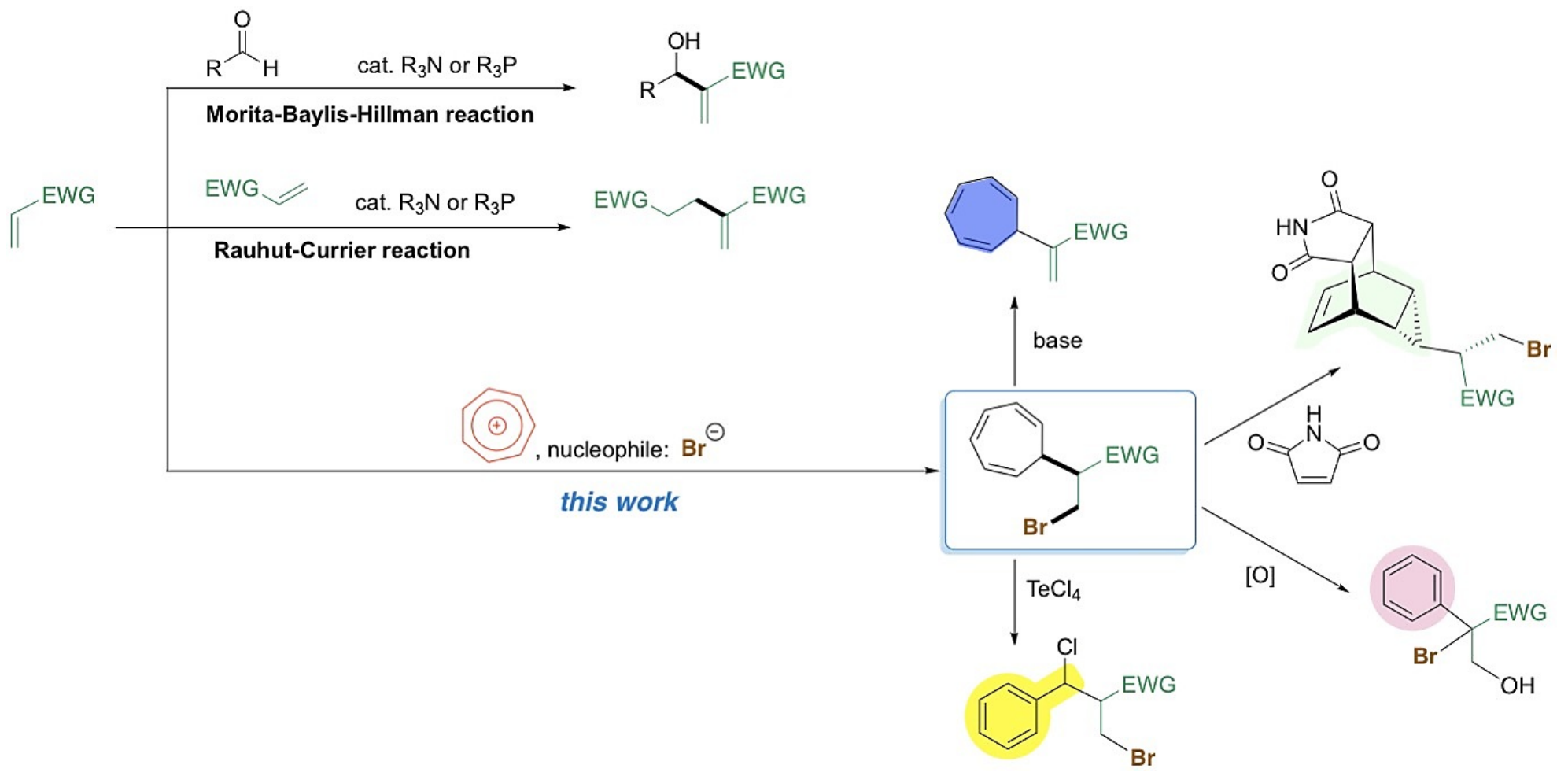




\title{
Halide Anion Activated Reactions of Michael Acceptors with Tropylium Ion
}

\author{
Mohanad A. Hussein, ${ }^{[\mathrm{a}]}$ Uyen P. N. Tran, ${ }^{[\mathrm{a}]}$ Vien T. Huynh,,${ }^{[\mathrm{b}]}$ Junming Ho, ${ }^{[\mathrm{a}]}$ Mohan \\ Bhadbhade, ${ }^{[\mathrm{c}]}$ Herbert Mayr ${ }^{[\mathrm{d}]}$ and Thanh V. Nguyen*[a]
}
[a] School of Chemistry, UNSW Sydney. E-mail: t.v.nguyen@unsw.edu.au
[b] School of Chemistry, University of Sydney
[c] Mark Wainwright Analytical Centre, UNSW Sydney
[d] Department Chemie, LMU München

\section{Supporting Information}

\section{Table of Contents}

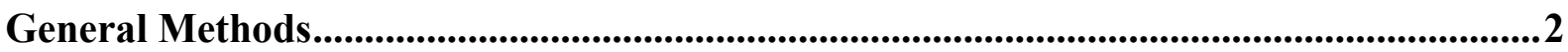

Table S1. Optimization Studies ......................................................................................3

General procedures for the reactions of Michael acceptors with tropylium salts..............4

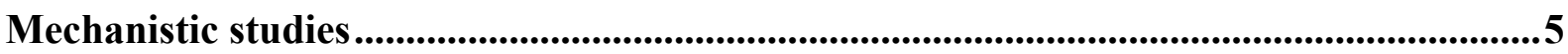

Characterization data of products in Table 1 .................................................................7

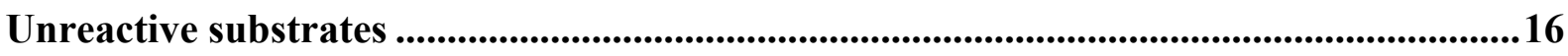

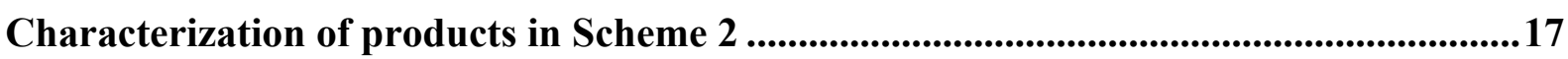

Characterization of products in Scheme 3 ................................................................19

Characterization data of products in Scheme 4 ..........................................................21

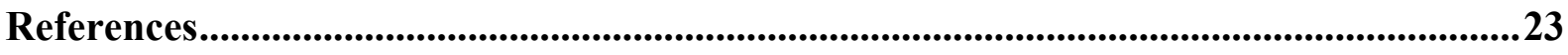

Computational Studies ............................................................................................................................24

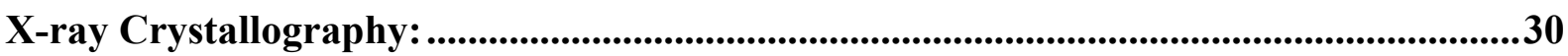

Table S2. Crystallographic Experimental details ............................................................31

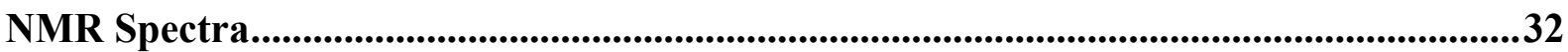




\section{General Methods}

Commercially available solvents and reagents were used as purchased unless otherwise noted. Tropylium bromide was prepared according to a literature procedure. ${ }^{1}$ Analytical thin layer chromatography was performed using aluminium plates precoated with silica gel $60 \mathrm{~F}_{254}(0.2$ $\mathrm{mm})$. Flash chromatography employed 230-400 mesh silica gel. Solvents used for chromatography are quoted as volume/volume ratios.

NMR spectroscopy was performed at $298 \mathrm{~K}$ using an Avance III HD $400\left(400.1 \mathrm{MHz},{ }^{1} \mathrm{H}\right.$; 100.6 MHz, $\left.{ }^{13} \mathrm{C}, 376.5 \mathrm{MHz},{ }^{19} \mathrm{~F}\right)$ or an Avance III $300\left(300 \mathrm{MHz},{ }^{1} \mathrm{H} ; 75 \mathrm{MHz},{ }^{13} \mathrm{C} ; 282.5\right.$ $\left.\mathrm{MHz},{ }^{19} \mathrm{~F}\right)$. Data is expressed in parts per million (ppm) downfield shift from tetramethylsilane with residual solvent as an internal reference $(\delta 7.26 \mathrm{ppm}$ for chloroform, $5.27 \mathrm{ppm}$ for dichloromethane) and is reported as position ( $\delta$ in ppm), multiplicity ( $\mathrm{s}=$ singlet, $\mathrm{d}=$ doublet, $\mathrm{t}=$ triplet, $\mathrm{q}=$ quartet, $\mathrm{m}=$ multiplet $)$, coupling constant $(J$ in $\mathrm{Hz})$ and integration (number of protons). ${ }^{13} \mathrm{C}$ NMR spectra were recorded at $298 \mathrm{~K}$ with complete proton decoupling. Data is expressed in parts per million (ppm) downfield shift relative to the internal reference ( $\delta 77.2 \mathrm{ppm}$ for the central peak of deuterated chloroform).

Infrared spectra were obtained on a ThermoNicolet Avatar 370 FT-IR spectrometer and are reported in wavenumbers $\left(\mathrm{cm}^{-1}\right)$. HRMS were performed at the Bioanalytical Mass Spectrometry Facility within the Mark Wainwright Analytical Centre at the University of New South Wales on an Orbitrap LTQ XL (Thermo Fisher Scientific, San Jose, CA, USA) ion trap mass spectrometer. 
Table S1. Optimization Studies

\begin{tabular}{cccccccc}
\hline & & & & \\
\hline
\end{tabular}

The reaction was optimized to obtain maximum yield of $\mathbf{4 a}$ with the reaction time being when the reaction did not progress any further as monitored by TLC and ${ }^{1} \mathrm{H}$ NMR.

Procedure A follows the conditions in entry 6 .

Procedure B follows the conditions in entry 11 . 
Procedure A with tropylium bromide

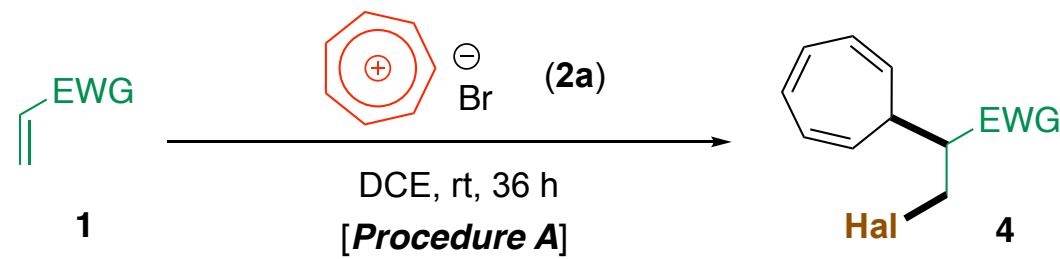

To a solution of alkene $(1,1.00 \mathrm{mmol})$ in 1,2-dichloroethane (DCE, $10 \mathrm{~mL})$ in a $25 \mathrm{~mL}$ round bottom flask was added tropylium bromide (2a, $1.05 \mathrm{mmol}, 1.05$ equiv.). The reaction mixture was stirred at room temperature for $36 \mathrm{~h}$ under $\mathrm{N}_{2}$ atmosphere. The reaction mixture was subsequently concentrated under reduced pressure. The residues were purified by flash column chromatography (silica-gel, hexanes/EtOAc $=1: 9$ ) to obtain product 4.

Procedure B with tropylium tetrafluoroborate and TMSHal

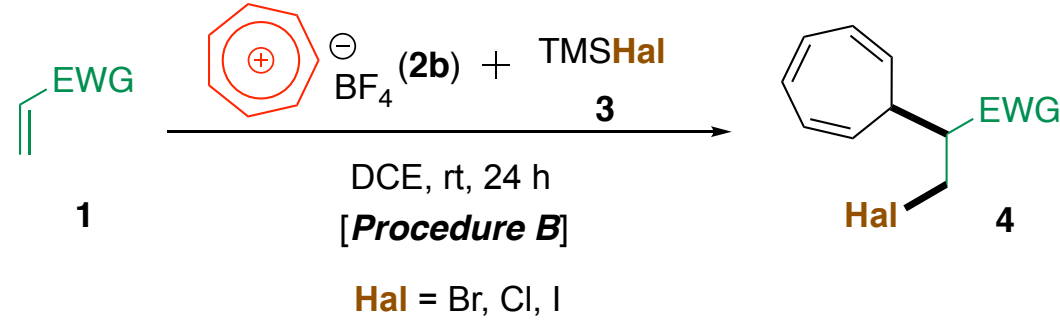

To a solution of alkene $(1,1.00 \mathrm{mmol})$ in DCE $(10 \mathrm{~mL})$ in a $25 \mathrm{~mL}$ round bottom flask was added tropylium tetrafluoroborate (2b, $1.05 \mathrm{mmol}, 1.05$ equiv.) followed by TMSHal $(\mathbf{3}, 1.05$ mmol, 1.05 eq.). The reaction mixture was stirred at room temperature for $24 \mathrm{~h}$ under $\mathrm{N}_{2}$ atmosphere. The reaction mixture was subsequently concentrated under reduced pressure. The residues were purified by flash column chromatography (silica-gel, hexanes/EtOAc $=1: 9$ ) to obtain product 4 . 


\section{Mechanistic studies}

NMR samples were prepared by dissolving $0.05 \mathrm{mmol}$ of tropylium tetrafluoroborate or $\mathrm{TMSBr}$ or a mixture of tropylium tetrafluoroborate and $\operatorname{TMSBr}(1: 1$ ratio on $0.05 \mathrm{mmol}$ scale) in $\mathrm{CD}_{3} \mathrm{CN}(1 \mathrm{~mL}) . \mathrm{CD}_{3} \mathrm{CN}$ was used instead of DCE for better shimming and locking in NMR experiments. ${ }^{1} \mathrm{H}$ and ${ }^{19} \mathrm{~F}$ NMR spectra were recorded immediately after sample preparation. They indicated that TMSF formation occurred instantaneously.

${ }^{1} \mathrm{H} \mathrm{CD}{ }_{3} \mathrm{CN}, 400 \mathrm{MHz}, 25^{\circ} \mathrm{C}$

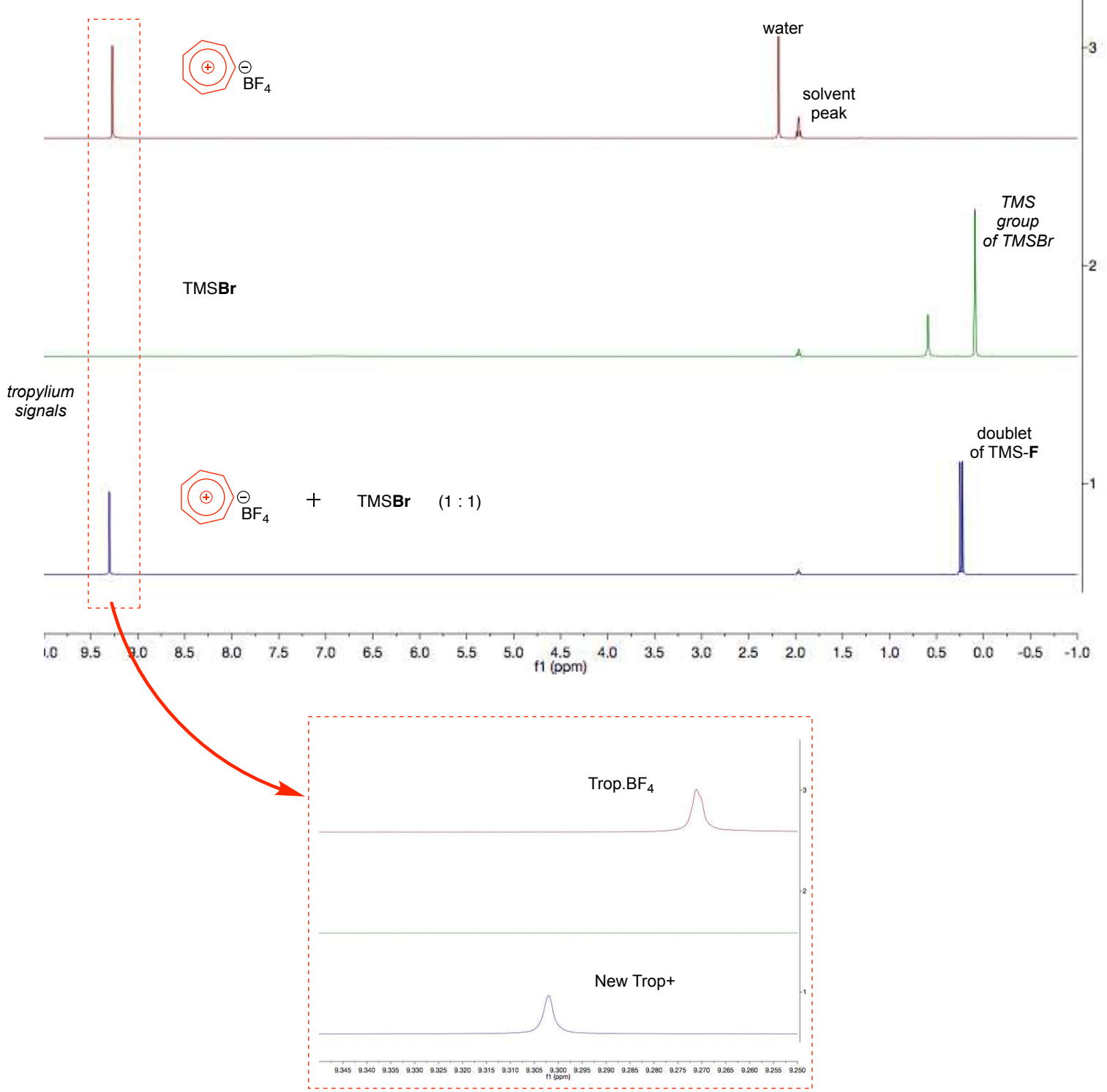

Figure S1a. Mechanistic ${ }^{1} \mathrm{H}$ NMR studies 


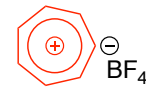

$\mathrm{BF}_{3}$ (with some $\mathrm{BF}_{3} \cdot \mathrm{Et}_{2} \mathrm{O}$ )

$\oplus \underset{\mathrm{BF}_{4}}{\ominus}+\mathrm{TMSBr} \quad(1: 1)$

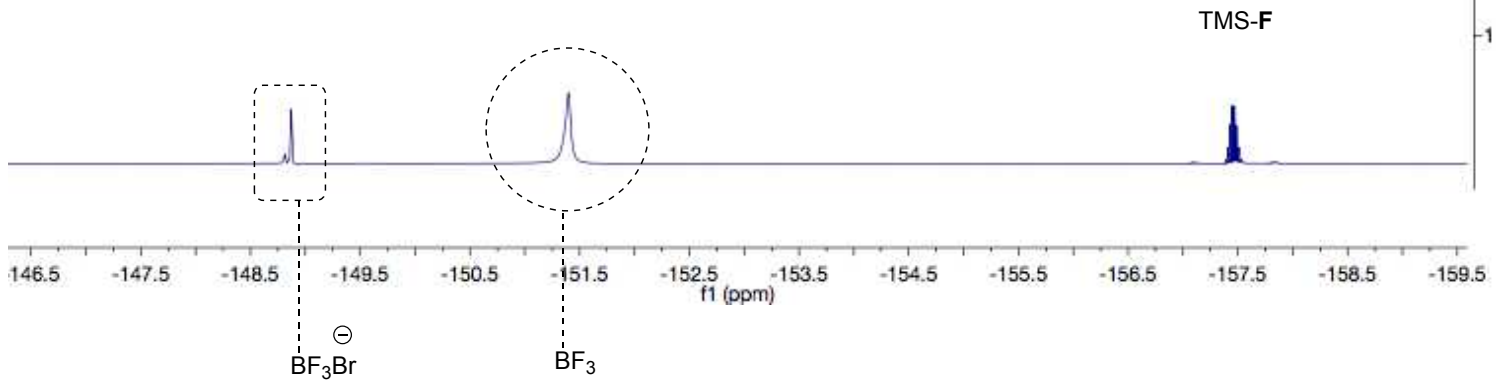

Figure S1b. Mechanistic ${ }^{1} \mathrm{H}$ NMR studies

From these studies, it can be concluded that tropylium tetrafluoroborate reacted with TMSBr to give a mixture of TMSF, tropylium ion and a mixture of $\mathrm{BF}_{3}, \mathrm{Br}^{\ominus}$ and $\stackrel{\ominus}{\stackrel{\ominus}{\mathrm{BF}_{3} \mathrm{Br}}}$

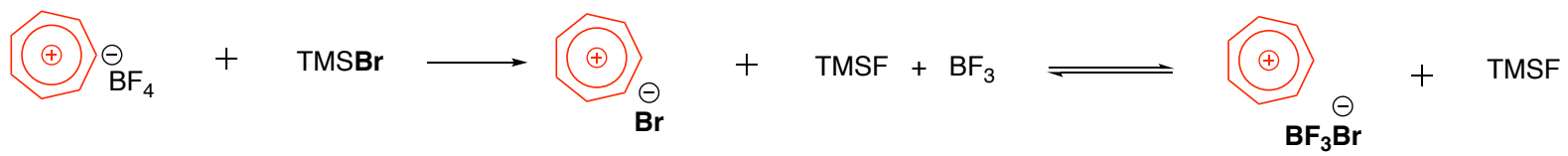




\section{Characterization data of products in Table 1}

Methyl 3-bromo-2-(cyclohepta-2,4,6-trien-1-yl)propanoate (compound 4a) Prepared by procedure B from (methyl acrylate 1a), tropylium tetrafluoroborate $\mathbf{2 b}$ and TMSBr $\mathbf{3}$ as anion source to give compound $\mathbf{4 a}$ as a colorless oil (192 $\mathrm{mg}, 75 \%$ yield).

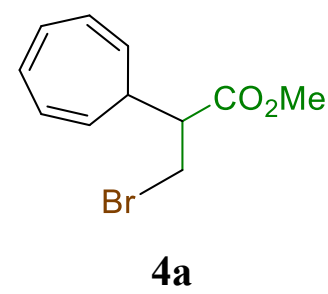

${ }^{1}$ H NMR (400 MHz, CDCl N $\delta$ 6.67-6.65 (m, 2H), 6.29-6.21 (m, 2H), 5.35 (dd, $J=9.6,6.5$ $\mathrm{Hz}, 1 \mathrm{H}), 5.29$ (dd, $J=9.6,6.5 \mathrm{~Hz}, 1 \mathrm{H}) 3.75(\mathrm{~s}, 3 \mathrm{H}), 3.65-3.56(\mathrm{~m}, 2 \mathrm{H}), 3.10$ (ddd, $J=9.7$, 8.0, $4.9 \mathrm{~Hz}, 1 \mathrm{H}), 2.28(\mathrm{~m}, 1 \mathrm{H}) \mathrm{ppm}$;

${ }^{13}$ C NMR (100 MHz, $\left.\mathbf{C D C l}_{3}\right) \delta 172.5,131.3,130.9,126.3,126.0,122.0,121.4,52.1,49.0$, 40.5, 31.0 ppm;

IR (KBr) 3016, 2950, 1734, $1435 \mathrm{~cm}^{-1}$;

HRMS (ESI+) m/z: $[\mathrm{M}+\mathrm{Na}]^{+}$Calcd. for $\mathrm{C}_{11} \mathrm{H}_{13} \mathrm{Br}_{1} \mathrm{O}_{2}+\mathrm{Na} 278.9997$ (100\%), 280.9976 (97.3\%); Found 278.9992 (100\%), 280.9967 (97.3\%).

Methyl 3-chloro-2-(cyclohepta-2,4,6-trien-1-yl)propanoate (compound number 4aCl): Prepared using the general procedure B from (methyl acrylate 1a), tropylium tetrafluoroborate $\mathbf{2 b}$ and $\mathrm{TMSCl} \mathbf{3 C l}$ as anion source to give the title compound as a colorless oil (117 $\mathrm{mg}, 55 \%$ yield).

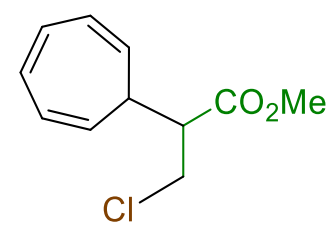

${ }^{1}$ H NMR (400 MHz, $\left.\mathbf{C D C l}_{3}\right) \delta$ 6.67-6.65 (m, 2H), 6.30-6.2 (m, 2H), $5.35(\mathrm{dd}, J=9.4,6.6$ $\mathrm{Hz}, 1 \mathrm{H}), 5.29(\mathrm{dd}, J=9.4,6.6 \mathrm{~Hz}, 1 \mathrm{H}), 3.76(\mathrm{~s}, 3 \mathrm{H}), 3.42-3.33(\mathrm{~m}, 2 \mathrm{H}), 3.00(\mathrm{td}, J=9.1,4.3$ $\mathrm{Hz}, 1 \mathrm{H}), 2.23(\mathrm{~m}, 1 \mathrm{H}) \mathrm{ppm}$;

${ }^{13}$ C NMR (100 MHz, CDCl $) \delta 172.9,131.4,131.0,126.4,126.1,122.3,121.5,52.3,49.3$, 42.2, $3.1 \mathrm{ppm}$.

IR (KBr) 3012, 2356, 2119, 1709, $1402 \mathrm{~cm}^{-1}$;

HRMS (ESI+) m/z: [M+Na $]^{+}$Calcd. for $\mathrm{C}_{11} \mathrm{H}_{13} \mathrm{Cl}_{1} \mathrm{O}_{2}+\mathrm{Na} 235.0502$; Found 235.0498. 
Methyl 2-(cyclohepta-2,4,6-trien-1-yl)-3-iodopropanoate (compound number 4aI): Prepared using the general procedure B from (methyl acrylate 1a), tropylium tetrafluoroborate 2b and TMSI 3I as anion source to give the title compound as a colorless oil (200 $\mathrm{mg}, 66 \%$ yield).

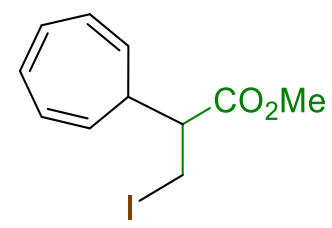

${ }^{1}$ H NMR (400 MHz, CDCl $)$ ) $\delta$ 6.76-6.61 (m, 2H), 6.36-6.17 (m, 2H), $5.37(\mathrm{dd}, J=9.5,6.5$ $\mathrm{Hz}, 1 \mathrm{H}), 5.31(\mathrm{dd}, J=9.5,6.5 \mathrm{~Hz}, 1 \mathrm{H}), 3.76(\mathrm{~s}, 3 \mathrm{H}), 3.42-3.33(\mathrm{~m}, 2 \mathrm{H}), 3.03(\mathrm{td}, J=9.1,4.4$ $\mathrm{Hz}, 1 \mathrm{H}), 2.25(\mathrm{~m}, 1 \mathrm{H}) \mathrm{ppm}$;

${ }^{13}$ C NMR (100 MHz, $\left.\mathbf{C D C l}_{3}\right) \delta 172.8,131.3,130.9,126.3,126.0,122.2,121.4,52.1,49.1$, 42.1, $3.0 \mathrm{ppm}$.

IR (KBr) 3014, 2949, 2848, 1731, $1434 \mathrm{~cm}^{-1}$;

HRMS (ESI+) m/z: $[\mathrm{M}+\mathrm{Na}]^{+}$Calcd. for $\mathrm{C}_{11} \mathrm{H}_{13} \mathrm{I}_{1} \mathrm{O}_{2}+\mathrm{Na}$ 326.9858; Found 326.9854 .

Ethyl 3-bromo-2-(cyclohepta-2,4,6-trien-1-yl)propanoate (compound 4b) and (compound 4b-H): Prepared using the general procedure B from (ethyl acrylate 1b), tropylium tetrafluoroborate $\mathbf{2 b}$ and $\mathrm{TMSBr} \mathbf{3}$ as anion source to give the title compound as a colorless oil (198 mg, 73\% yield).

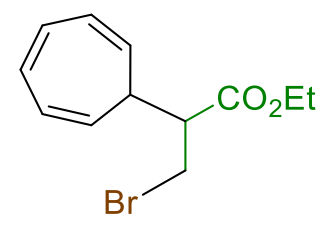

${ }^{1}$ H NMR (400 MHz, CDCl $)$ ) $\delta$ 6.75-6.61 (m, 2H), 6.36-6.17 (m, 2H), $5.39(\mathrm{dd}, J=9.6,6.4$ $\mathrm{Hz}, 1 \mathrm{H}), 5.33$ (dd, $J=9.6,6.4, \mathrm{~Hz}, 2 \mathrm{H}), 4.26(\mathrm{qd}, J=7.1,0.9 \mathrm{~Hz}, 2 \mathrm{H}), 3.72-3.55(\mathrm{~m}, 2 \mathrm{H})$, $3.10(\mathrm{ddd}, J=9.4,8.4,4.7 \mathrm{~Hz}, 1 \mathrm{H}), 2.30(\mathrm{~m}, 1 \mathrm{H}), 1.31(\mathrm{t}, J=7.0 \mathrm{~Hz}, 3 \mathrm{H}) \mathrm{ppm}$;

${ }^{13}$ C NMR (100 MHz, $\left.\mathbf{C D C l}_{3}\right) \delta 172.2,131.5,131.0,126.3,126.4,122.2,121.6,61.2,49.2$, 40.7, 31.2, $14.5 \mathrm{ppm}$.

IR (KBr) 3015, 2979, 1729, $1426 \mathrm{~cm}^{-1}$;

HRMS (ESI+) $\mathbf{m} / \mathbf{z}:[\mathrm{M}+\mathrm{Na}]^{+}$Calcd. for $\mathrm{C}_{12} \mathrm{H}_{15} \mathrm{Br}_{1} \mathrm{O}_{2}+\mathrm{Na} 293.0153$ (100\%), 295.0133 (97.3\%); Found 293.0145 (100\%), 294.0127 (97.3\%). 
n-Butyl 3-bromo-2-(cyclohepta-2,4,6-trien-1-yl)propanoate (compound number 4c): Prepared using the general procedure B from (n-butyl acrylate 1c), tropylium tetrafluoroborate $\mathbf{2 b}$ and $\mathrm{TMSBr} \mathbf{3}$ as anion source to give the title compound as a colorless oil (202 mg, 66\% yield).

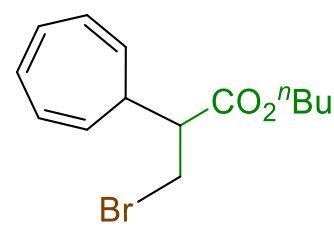

${ }^{1}$ H NMR (400 MHz, CDCl $) \delta$ 6.76-6.61 (m, 2H), 6.36-6.16 (m, 2H), $5.38(\mathrm{dd}, J=9.6,6.4$ $\mathrm{Hz}, 1 \mathrm{H}), 5.32$ (dd, $J=9.6,6.4 \mathrm{~Hz}, 1 \mathrm{H}), 4.22-4.12(\mathrm{~m}, 2 \mathrm{H}), 3.65-3.56$ (m, 2H), 3.07 (ddd, $J=$ 9.5, 8.1, $4.9 \mathrm{~Hz}, 1 \mathrm{H}), 2.26(\mathrm{~m}, 1 \mathrm{H}), 1.68-1.60(\mathrm{~m}, 2 \mathrm{H}), 1.44-1.35(\mathrm{~m}, 2 \mathrm{H}), 0.93(\mathrm{t}, J=7.4 \mathrm{~Hz}$, 3H) ppm;

${ }^{13}$ C NMR (100 MHz, $\left.\mathbf{C D C l}_{3}\right) \delta 172.3,131.5,131.0,126.4,126.1,122.3,121.6,65.1,49.3$, 40.7, 31.2, 30.8, 19.3, $13.8 \mathrm{ppm}$.

IR (KBr) 3017, 2958, 2871, 1730, 1617, 1461, $1427 \mathrm{~cm}^{-1}$;

HRMS (ESI+) m/z: $[\mathrm{M}+\mathrm{Na}]^{+}$Calcd. for $\mathrm{C}_{14} \mathrm{H}_{19} \mathrm{Br}_{1} \mathrm{O}_{2}+\mathrm{Na} 321.0466$ (100\%), 323.0440 (97.3\%); Found 321.0462 (100\%), 323.0446 (97.3\%).

Isobutyl 3-bromo-2-(cyclohepta-2,4,6-trien-1-yl)propanoate (compound 4d): Prepared using the general procedure B from (isobutyl acrylate 1d), tropylium tetrafluoroborate $\mathbf{2 b}$ and TMSBr 3 as anion source to give the title compound as a colorless oil (211 mg, 71\% yield).

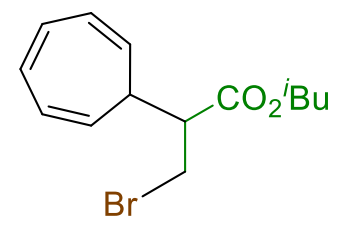

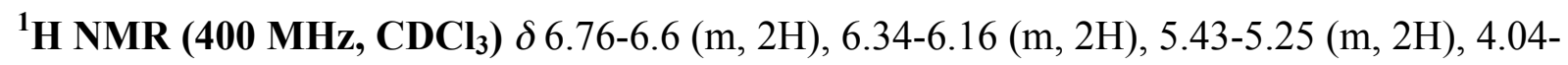
$3.86(\mathrm{~m}, 2 \mathrm{H}), 3.7-3.57$ (m, 2H), 3.09 (ddd, $J=9.5,7.9,5.0 \mathrm{~Hz}, 1 \mathrm{H}), 2.27$ (m, 1H), 1.97 (dp, $J$ $=13.4,6.7 \mathrm{~Hz}, 1 \mathrm{H}) 0.95(\mathrm{~d}, J=6.7 \mathrm{~Hz}, 6 \mathrm{H}) \mathrm{ppm}$;

${ }^{13} \mathbf{C}$ NMR (100 MHz, $\left.\mathbf{C D C l}_{3}\right) \delta 172.3,131.4,131.0,126.4,126.1,122.3,121.6,71.3,49.3$, 40.7, 31.2, 27.9, $19.3 \mathrm{ppm}$.

IR (KBr) 2955, 2921, 2852, 2359, 2341, 1734, $1456 \mathrm{~cm}^{-1}$;

HRMS (ESI+) m/z: $[\mathrm{M}+\mathrm{Na}]^{+}$Calcd. for $\mathrm{C}_{14} \mathrm{H}_{19} \mathrm{Br}_{1} \mathrm{O}_{2}+\mathrm{Na} 321.0466$ (100\%), 323.0440 (97.3\%); Found 321.0459 (100\%), 321.0438 (97.3\%) 
3-Bromo-2-(cyclohepta-2,4,6-trien-1-yl)propanoic acid (compound 4e/4g): Prepared using the general procedure B from ( $t$-butyl acrylate 1e or isobornyl acrylate 1g), tropylium tetrafluoroborate $\mathbf{2 b}$ and TMSBr $\mathbf{3}$ as anion source to give the title compound as a colorless oil (178 mg, 73\% yield with 1 e or $183 \mathrm{mg}, 75 \%$ with $\mathbf{1 g}$ ).

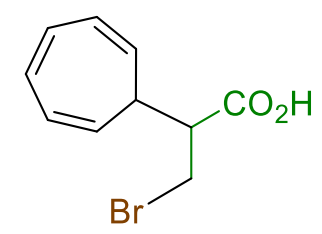

${ }^{1}$ H NMR (400 MHz, CDCl $) \delta 6.77-6.63(\mathrm{~m}, 2 \mathrm{H}), 6.38-6.25(\mathrm{~m}, 2 \mathrm{H}), 5.45(\mathrm{dd}, J=9.6$, $6.5 \mathrm{~Hz}, 1 \mathrm{H}), 5.36(\mathrm{dd}, J=9.5,6.7 \mathrm{~Hz}, 1 \mathrm{H}), 3.65$ (m, 2H), $3.13(\mathrm{dt}, J=9.4,6.2 \mathrm{~Hz}, 1 \mathrm{H}), 2.42$ (m, 1H) ppm.

${ }^{13}$ C NMR (100 MHz, CDCl $) \delta 177.4,131.3,131.0,126.7,126.3,122.2,48.4,40.3,30.6$ ppm.

IR (KBr) 3015, 2926, 2656, 1704, 1433, $1401 \mathrm{~cm}^{-1}$;

HRMS (ESI+) m/z: $[\mathrm{M}+\mathrm{Na}]^{+}$Calcd. for $\mathrm{C}_{10} \mathrm{H}_{11} \mathrm{Br}_{1} \mathrm{O}_{2}+\mathrm{Na} 264.9840$ (100\%), 266.9812 (97.3\%); Found 264.9833 (100\%), 266.9820 (97.3\%).

2-Phenoxyethyl 3-bromo-2-(cyclohepta-2,4,6-trien-1-yl)propanoate (compound number 4f) Prepared using the general procedure B from (ethylene glycol phenyl ether acrylate 1f), tropylium tetrafluoroborate $\mathbf{2 b}$ and TMSBr $\mathbf{3}$ as anion source to give the title compound as a colorless oil (297 mg, 82\% yield).

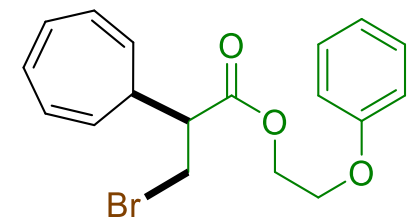

${ }^{1}$ H NMR (400 MHz, $\left.\mathbf{C D C l}_{3}\right) \delta 7.36-7.26(\mathrm{~m}, 3 \mathrm{H}), 7.03-6.89(\mathrm{~m}, 3 \mathrm{H}), 6.70-6.62(\mathrm{~m}$, $2 \mathrm{H}), 6.31-6.24(\mathrm{~m}, 1 \mathrm{H}), 6.22-6.14(\mathrm{~m}, 1 \mathrm{H}), 5.39(\mathrm{dd}, J=9.5,6.5 \mathrm{~Hz}, 1 \mathrm{H}), 5.32(\mathrm{dd}, J=$ 9.5, 6.5 Hz, 1H), 4.63 - 4.49 (m, 2H), 4.28 - 4.16 (m, 2H), 3.71 - 3.57 (m, 2H), 3.16 (ddd, $J$ $=9.5,8.2,4.8 \mathrm{~Hz}, 1 \mathrm{H}), 2.31(\mathrm{~m}, 1 \mathrm{H}) \mathrm{ppm}$;

${ }^{13}$ C NMR (100 MHz, $\left.\mathbf{C D C l}_{3}\right) \delta 172.2,131.5,131.0,126.4,129.7,126.5,126.2,122.1,121.5$, $114.8,65.8,63.4,49.0,40.7,31.0 \mathrm{ppm}$.

IR (KBr) 2925, 1734, 2871, 1598, 1587, 1495, 1455, $1404 \mathrm{~cm}^{-1}$;

HRMS (ESI+) $\mathbf{m} / \mathbf{z}:[\mathrm{M}+\mathrm{Na}]^{+}$Calcd. for $\mathrm{C}_{18} \mathrm{H}_{19} \mathrm{Br}_{1} \mathrm{O}_{3}+\mathrm{Na} 385.0408$ (100\%), 387.0406 (97.3\%); Found 385.0418 (100\%), 387.0395 (97.3\%). 
((Oxybis(ethane-2,1-diyl))bis(oxy))bis(ethane-2,1-diyl)bis(3-bromo-2-(cyclohepta-2,4,6trien-1-yl)propanoate) (compound number 4h): Prepared using the general procedure B from (tetra(ethylene glycol) diacrylate $\mathbf{1 h}$ ), tropylium tetrafluoroborate $\mathbf{2 b}$ and TMSBr $\mathbf{3}$ as anion source to give the title compound as a yellow oil (405 mg, 63\% yield).

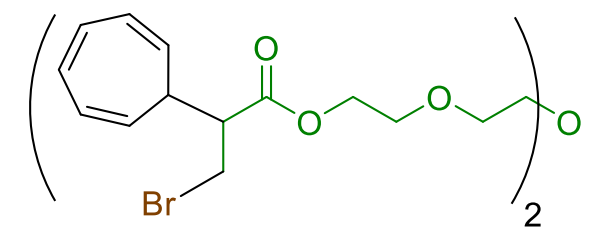

${ }^{1}$ H-NMR (300 MHz CDCl 3$) \delta 6.69-6.62(\mathrm{~m}, 2 \mathrm{H}), 6.31-6.18(\mathrm{~m}, 2 \mathrm{H}), 5.42-5.26(\mathrm{~m}, 3 \mathrm{H})$, $4.37-4.29$ (m, 2H), $3.74-3.56(\mathrm{~m}, 4 \mathrm{H}), 3.64(\mathrm{~s}, 4 \mathrm{H}), 3.12(\mathrm{ddd}, J=9.4,8.1,4.8 \mathrm{~Hz}, 1 \mathrm{H})$, $2.28(\mathrm{~m}, 1 \mathrm{H}) \mathrm{ppm}$.

${ }^{13}$ C-NMR (75 MHz $\mathbf{C D C l}_{3}$ ) $\delta$ 172.0, 131.3, 130.9, 126.3, 126.0, 122.1, 121.4, 70.7, 70.6, 69.1, 64.0, 48.9, 40.5, $31.1 \mathrm{ppm}$.

IR (KBr): 2870, 1720, 1635, 1522, $1406 \mathrm{~cm}^{-1}$;

HRMS (ESI+) $\mathbf{m} / \mathbf{z}[\mathrm{M}+\mathrm{Na}]^{+}$Calcd. for $\mathrm{C}_{28} \mathrm{H}_{36} \mathrm{Br}_{2} \mathrm{O}_{7}+\mathrm{Na} 667.0705$ (100\%), 665.0724 (97.3\%); Found 667.0700 (100\%), 665.0725 (97.3\%).

Vinyl 3-bromo-2-(cyclohepta-2,4,6-trien-1-yl)propanoate (compound number 4i): Prepared using the general procedure B from (vinyl acrylate 1i), tropylium tetrafluoroborate $\mathbf{2 b}$ and $\mathrm{TMSBr} 3$ as anion source to give the title compound as a colorless oil (206 mg, 77\% yield).

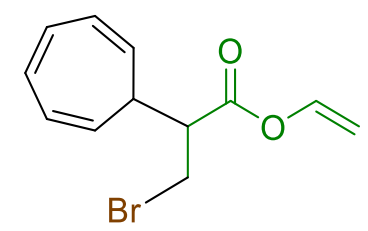

${ }^{1}$ H NMR (400 MHz, CDCl $) \delta 7.32(\mathrm{dd}, J=13.9,6.3 \mathrm{~Hz}, 1 \mathrm{H}), 6.73-6.66(\mathrm{~m}, 2 \mathrm{H}), 6.37$ $6.23(\mathrm{~m}, 2 \mathrm{H}), 5.41(\mathrm{dd}, J=9.6,6.7 \mathrm{~Hz}, 1 \mathrm{H}), 5.35(\mathrm{dd}, J=9.6,6.7 \mathrm{~Hz}, 1 \mathrm{H}), 4.98(\mathrm{dd}, J=13.9$, $1.8 \mathrm{~Hz}, 1 \mathrm{H}), 4.67$ (dd, $J=6.3,1.8 \mathrm{~Hz}, 1 \mathrm{H}), 3.71-3.57$ (m, 2H), 3.16 (ddd, $J=9.5,7.6,5.0$ $\mathrm{Hz}, 1 \mathrm{H}), 2.45$ (m, 1H) ppm.

${ }^{13}$ C NMR (100 MHz, $\left.\mathbf{C D C l}_{3}\right) \delta 169.4,141.1,131.5,131.0,126.7,126.4,122.0,121.2,98.8$, 48.5, 40.4, $30.5 \mathrm{ppm}$.

IR (KBr) 3018, 1746, 1645, 1357, 1254, $1142 \mathrm{~cm}^{-1}$;

HRMS (ESI+) m/z: $[\mathrm{M}+\mathrm{Na}]^{+}$Calcd. for $\mathrm{C}_{12} \mathrm{H}_{13} \mathrm{Br}_{1} \mathrm{O}_{2}+\mathrm{Na} 290.9997$ (100\%), 292.9976 (97.3\%); Found 290.9994 (100\%), 292.9972 (97.3\%). 
Prop-2-yn-1-yl 3-bromo-2-(cyclohepta-2,4,6-trien-1-yl)propanoate (compound 4j): Prepared using the general procedure B from (propargyl acrylate $\mathbf{1 j}$ ), tropylium tetrafluoroborate $\mathbf{2 b}$ and TMSBr $\mathbf{3}$ as anion source to give the title compound as a colorless oil (218 mg, 78\% yield).

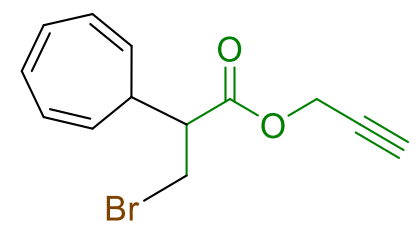

${ }^{1}$ H NMR (400 MHz, $\left.\mathbf{C D C l}_{3}\right) \delta 6.75-6.63(\mathrm{~m}, 2 \mathrm{H}), 6.35-6.22(\mathrm{~m}, 2 \mathrm{H}), 5.41(\mathrm{dd}, J=9.5$, $6.6 \mathrm{~Hz}, 1 \mathrm{H}), 5.33(\mathrm{dd}, J=9.5,6.6 \mathrm{~Hz}, 1 \mathrm{H}), 4.79(\mathrm{~d}, J=2.5 \mathrm{~Hz}, 1 \mathrm{H}), 4.77(\mathrm{~d}, J=2.5 \mathrm{~Hz}, 1 \mathrm{H})$, $3.70-3.58(\mathrm{~m}, 2 \mathrm{H}), 3.15(\mathrm{ddd}, J=9.5,7.9,4.9 \mathrm{~Hz}, 1 \mathrm{H}), 2.52$ (t, $J=2.5 \mathrm{~Hz}, 1 \mathrm{H}), 2.39$ (m, 1H) $\mathrm{ppm}$.

${ }^{13}$ C NMR (100 MHz, CDCl$\left._{3}\right) \delta 171.5,131.5,126.6,122.1,121.3,75.4,52.6,48.6,40.6,30.8$ ppm.

IR (KBr) 3292, 3017, 1739, $1432 \mathrm{~cm}^{-1}$;

HRMS (ESI+) $\mathbf{m} / \mathbf{z}:[\mathrm{M}+\mathrm{Na}]^{+}$Calcd. for $\mathrm{C}_{13} \mathrm{H}_{13} \mathrm{Br}_{1} \mathrm{O}_{2}+\mathrm{Na} 302.9997$ (100\%), 304.9970 (97.3\%); Found 302.9993 (100\%), 304.9976 (97.3\%).

3-Bromo-2-(cyclohepta-2,4,6-trien-1-yl)propanamide (compound 4k): Prepared using the general procedure B from (acrylamide 1k), tropylium tetrafluoroborate $\mathbf{2 b}$ and TMSBr $\mathbf{3}$ as anion source to give the title compound as a white solid (220 mg, 91\% yield).

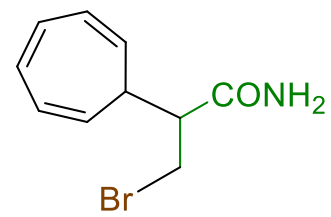

${ }^{1}$ H NMR (400 MHz, CDCl $) \delta 6.72-6.65(\mathrm{~m}, 2 \mathrm{H}), 6.36-6.24(\mathrm{~m}, 2 \mathrm{H}), 6.02(\mathrm{bs}, 1 \mathrm{H}), 5.76$ (bs, 1H), $5.42(\mathrm{dd}, J=9.6,6.7 \mathrm{~Hz}, 1 \mathrm{H}), 5.33(\mathrm{dd}, J=9.6,6.7 \mathrm{~Hz}, 1 \mathrm{H}), 3.69-3.56(\mathrm{~m}, 2 \mathrm{H})$, $2.82(\mathrm{td}, J=9.8,3.8 \mathrm{~Hz}, 1 \mathrm{H}), 2.39(\mathrm{~m}, 1 \mathrm{H}) \mathrm{ppm}$;

${ }^{13}$ C NMR (100 MHz, CDCl $) \delta 174.2,131.2,131.1,126.7,126.3,122.5,121.6,49.6,41.0$, $31.5 \mathrm{ppm}$.

IR (KBr) 3382, 3187, 3009, 2915, 1654, $1434 \mathrm{~cm}^{-1}$;

HRMS (ESI+) m/z: $[\mathrm{M}+\mathrm{Na}]^{+}$Calcd. for $\mathrm{C}_{10} \mathrm{H}_{12} \mathrm{Br}_{1} \mathrm{~N}_{1} \mathrm{O}_{1}+\mathrm{Na} 264.0000$ (100\%), 265.9979 (97.3\%); Found 263.9997 (100\%), 265.9973 (97.3\%). 
3-Bromo-2-(cyclohepta-2,4,6-trien-1-yl)- $N, N$-dimethylpropanamide (compound $4 \mathrm{l}$ ) and 3-Bromo- $N, N$-dimethylpropanamide ${ }^{2}$ (compound 4l-H): Prepared using the general procedure B from ( $N, N$-dimethylacrylamide $\mathbf{1 1})$, tropylium tetrafluoroborate $\mathbf{2 b}$ and TMSBr $\mathbf{3}$ as anion source to give the title compound as a yellowish oil (214 $\mathrm{mg}, 79 \%$ yield) and compound $\mathbf{4 l - H}$ as a yellowish oil (36 $\mathrm{mg}, 20 \%$ yield).

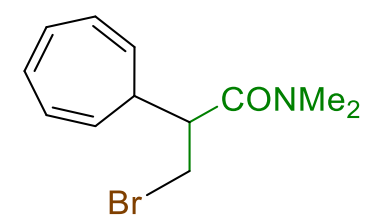

${ }^{1}$ H NMR (400 MHz, CDCl $) \delta 6.70-6.63(\mathrm{~m}, 2 \mathrm{H}), 6.33(\mathrm{~m}, 1 \mathrm{H}), 6.23(\mathrm{~m}, 1 \mathrm{H}), 5.41(\mathrm{dd}, J$ $=9.7,7.2 \mathrm{~Hz}, 1 \mathrm{H}), 5.33(\mathrm{dd}, J=9.7,7.2 \mathrm{~Hz}, 1 \mathrm{H}), 3.67(\mathrm{dd}, J=10.9,9.4 \mathrm{~Hz}, 1 \mathrm{H}), 3.54(\mathrm{dd}, J$ =9.4, 3.4 Hz, 1H), $3.36(\mathrm{td}, J=10.5,3.4 \mathrm{~Hz}, 1 \mathrm{H}), 3.04(\mathrm{~s}, 3 \mathrm{H}), 3.03(\mathrm{~s}, 3 \mathrm{H}), 2.72(\mathrm{~m}, 1 \mathrm{H})$ ppm.

${ }^{13}$ C NMR (100 MHz, $\left.\mathbf{C D C l}_{3}\right) \delta 172.6,131,0,130.8,126.9,126.5,123.2,122.9,43.3,41.9$, $37.9,36.1,32.7 \mathrm{ppm}$.

IR (KBr) 3454, 2931, 2359, 1629, $1496 \mathrm{~cm}^{-1}$;

HRMS (ESI+) m/z: $[\mathrm{M}+\mathrm{Na}]^{+}$Calcd. for $\mathrm{C}_{12} \mathrm{H}_{16} \mathrm{Br}_{1} \mathrm{~N}_{1} \mathrm{O}_{1}+\mathrm{Na} 292.0313$ (100\%), 294.0292 (97.3\%); Found 292.0307 (100\%), $294.0286(97.3 \%)$.

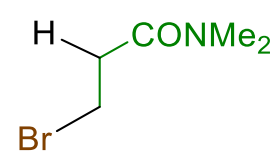

4l-H

${ }^{1}$ H NMR (400 MHz, CDCl $) \delta 3.67$ (t, $\left.J=7.2 \mathrm{~Hz}, 2 \mathrm{H}\right), 3.04$ (s, 3H), 2.99 (s, 3H), 2.93 (t, $J$ $=7.2 \mathrm{~Hz}, 2 \mathrm{H}) \mathrm{ppm}$;

${ }^{13}$ C NMR (100 MHz, CDCl $) \delta 170.2,37.3,36.8,35.6,27.5$ ppm.

IR (KBr) 3015, 2924, 2359, 1715, 1636, 1493, $1420 \mathrm{~cm}^{-1}$;

HRMS (ESI+) m/z: $[\mathrm{M}+\mathrm{Na}]^{+}$Calcd. for $\mathrm{C}_{5} \mathrm{H}_{10} \mathrm{Br}_{1} \mathrm{~N}_{1} \mathrm{O}_{1}+\mathrm{Na} 201.9838$ (100\%), 203.9818 (97.3\%); Found 201.9843 (100\%), 201.9823 (97.3\%).

3-Bromo-2-(cyclohepta-2,4,6-trien-1-yl)-1-morpholinopropan-1-one (compound 4m): Prepared using the general procedure B from (4-acryloylmorpholine 1m), tropylium 
tetrafluoroborate $\mathbf{2 a}$ and TMSBr $\mathbf{3}$ as anion source to give the title compound as a colorless oil (220 mg, 71\% yield).

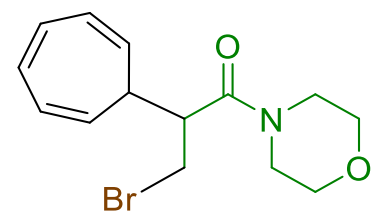

${ }^{1}$ H-NMR (300 MHz CDCl 3$) \delta 6.67-6.56(\mathrm{~m}, 2 \mathrm{H}), 6.30(\mathrm{ddd}, J=9.8,4.8,1.5 \mathrm{~Hz}, 1 \mathrm{H}), 6.22$ (ddd, $J=9.8,4.8,1.5 \mathrm{~Hz}, 1 \mathrm{H}), 5.39$ (dd, $J=9.7,7.4 \mathrm{~Hz}, 1 \mathrm{H}), 5.30$ (dd, $J=9.7,7.4 \mathrm{~Hz}, 1 \mathrm{H}$ ), $3.86-3.75$ (m, 1H), $3.72-3.42(\mathrm{~m}, 8 \mathrm{H}), 3.40-3.29(\mathrm{~m}, 1 \mathrm{H}), 3.23(\mathrm{td}, J=10.6,3.3 \mathrm{~Hz}, 1 \mathrm{H})$, $2.83(\mathrm{~m}, 1 \mathrm{H}) \mathrm{ppm}$;

${ }^{13}$ C-NMR (75 MHz CDCl $) \delta 171.1,131.1,130.6,127.1,126.6,123.1,122.8,67.1,66.8$, 46.6, 42.5, 42.1, 41.8, $32.5 \mathrm{ppm}$.

IR (KBr): 3013, 2961, 2918, 2853, 1632, $1437 \mathrm{~cm}^{-1}$;

HRMS (ESI+) $\mathbf{m} / \mathbf{z}[\mathrm{M}+\mathrm{Na}]^{+}$Calcd. for $\mathrm{C}_{14} \mathrm{H}_{18} \mathrm{Br}_{1} \mathrm{O}_{2}+\mathrm{Na} 334.0419$ (100\%), 336.0398 (97.3\%); Found 334.0412 (100\%), 336.0393 (97.3\%).

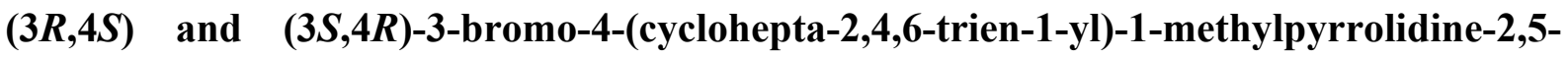
dione (compound 4n): Prepared using the general procedure B from (1-methyl-1H-pyrrole2,5-dione $\mathbf{1 n}$ ), tropylium tetrafluoroborate $\mathbf{2 b}$ and TMSBr $\mathbf{3}$ as anion source to give the title compound as a yellowish oil (214 mg, 76\% yield). The small $\mathrm{H}-\mathrm{H}$ coupling constant of $(3 \mathrm{H}, 4 \mathrm{H})$ indicated that the compounds was formed with exclusive anti-selectivity to give a mixture of $(3 R, 4 S)$ and $(3 S, 4 R)$ diastereoisomers.

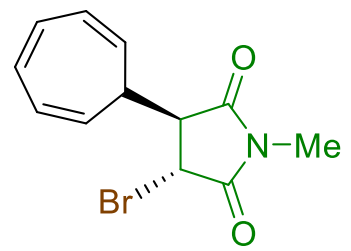

${ }^{1}$ H-NMR (300 MHz CDCl $) \delta$ 6.74-6.70 (m, 2H), 6.37-6.28 (m, 2H), 5.52-5.27 (m, 2H), 4.49 $(\mathrm{d}, J=3.7 \mathrm{~Hz}, 1 \mathrm{H}), 3.47-3.40(\mathrm{dd}, J=7.5,3.7 \mathrm{~Hz}, 1 \mathrm{H}), 3.07$ (s, 3H), 2.15-2.07 (m, 1H) ppm; ${ }^{13}$ C-NMR (75 MHz CDCl 3 ) $8175.7,173.3,132.3,131.8,127.8,126.9,122.2,120.3,53.2$, 41.2, 40.4, $26.3 \mathrm{ppm}$;

IR (KBr): 3015, 2940, 1703, $1431 \mathrm{~cm}^{-1}$;

HRMS (ESI+) $\mathbf{m} / \mathbf{z}[\mathrm{M}+\mathrm{Na}]^{+}$Calcd. for $\mathrm{C}_{12} \mathrm{H}_{12} \mathrm{BrNO}_{2}+\mathrm{Na} 303.9949$ (100\%), 305.9929 (97.3\%); Found 303.9941(100\%), 305.9922 (97.3\%). 
$(3 R, 4 S)$ and $(3 S, 4 R)-3$-bromo-1,4-di(cyclohepta-2,4,6-trien-1-yl)pyrrolidine-2,5-dione (compound 4o): Prepared using the general procedure B from (maleimide 10), tropylium tetrafluoroborate $\mathbf{2 b}$ ( 2 equiv) and TMSBr 3 (2 equiv) as anion source to give the title compound as white crystals (240 mg, 67\% yield). The small H-H coupling constant of $(3 \mathrm{H}, 4 \mathrm{H})$ indicated that the compounds was formed with exclusive anti-selectivity to give a mixture of $(3 R, 4 S)$ and $(3 S, 4 R)$ diastereoisomers.

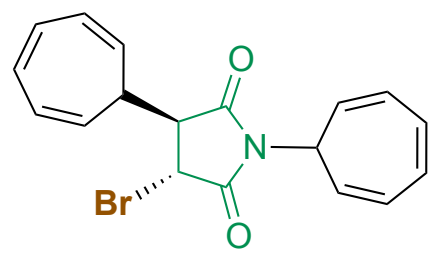

${ }^{1}$ H NMR (400 MHz, CDCl $)_{3} \delta$ 6.77-6.66 (m, 4H), 6.37-6.19 (m, 4H), 5.51-5.32 (m, 4H), $4.55(\mathrm{~d}, J=3.4 \mathrm{~Hz}, 1 \mathrm{H}), 4.17(\mathrm{tt}, J=3.4,1.5 \mathrm{~Hz}, 1 \mathrm{H}), 3.49$ (dd, $J=8.2,3.4 \mathrm{~Hz}, 1 \mathrm{H}), 2.20$ $2.14(\mathrm{~m}, 1 \mathrm{H}) \mathrm{ppm}$;

${ }^{13}$ C NMR (100 MHz, $\left.\mathbf{C D C l}_{3}\right) \delta 174.8,172.5,131.9,131.7,131.6,131.4,127.5,126.6,124.8$, $124.7,121.9,121.7,119.8,52.6,51.2,40.9,40.0 \mathrm{ppm}$.

IR (KBr) 3015, 2925, 2854, 1785, 1711, $1439 \mathrm{~cm}^{-1}$;

HRMS (ESI+) m/z: $[\mathrm{M}+\mathrm{Na}]^{+}$Calcd. for $\mathrm{C}_{18} \mathrm{H}_{16} \mathrm{Br}_{1} \mathrm{~N}_{1} \mathrm{O}_{2}+\mathrm{Na} 380.0262$ (100\%), 382.0242 (97.3\%); Found 380.0258 (100\%), 382.0239 (97.3\%).

1-Bromo-2-(cyclohepta-2,4,6-trien-1-yl)pentan-3-one (compound 4p): Prepared using the general procedure B from (1-penten-3-one 1p), tropylium tetrafluoroborate $\mathbf{2 b}$ and TMSBr $\mathbf{3}$ as anion source to give the title compound as a colorless oil (164 mg, 64\% yield).

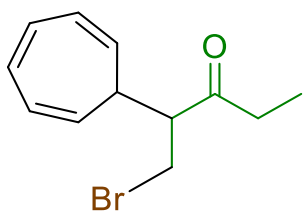

${ }^{1}$ H-NMR (300 MHz CDCl $) \delta 6.69-6.61(\mathrm{~m}, 2 \mathrm{H}), 6.33-6.18(\mathrm{~m}, 2 \mathrm{H}), 5.33-5.21(\mathrm{~m}, 2 \mathrm{H})$, $3.61-3.48(\mathrm{~m}, 2 \mathrm{H}), 3.21(\mathrm{~m}, 1 \mathrm{H}), 2.62-2.40(\mathrm{~m}, 3 \mathrm{H}), 1.06$ (t, $J=7.2 \mathrm{~Hz}, 3 \mathrm{H}) \mathrm{ppm}$;

${ }^{13}$ C-NMR (75 MHz CDCl $) \delta 211.5,131.2,131.1,126.8,126.5,122.5,121.6,53.5,40.9$, 38.1, 31.0, $7.3 \mathrm{ppm}$

IR (KBr): 3010, 2945, 2923, 2853, 2359, 1714, 1617, $1456 \mathrm{~cm}^{-1}$;

HRMS (ESI+) $\mathbf{m} / \mathbf{z}[\mathrm{M}+\mathrm{Na}]^{+}$Calcd. for $\mathrm{C}_{12} \mathrm{H}_{15} \mathrm{Br}_{1} \mathrm{O}_{1}+\mathrm{Na} 277.0204$ (100\%), 277.0199 (97.3\%); Found 277.0199 (100\%), 277.0177 (97.3\%). 


\section{Unreactive substrates}<smiles>C=C(C)C(=O)OC</smiles><smiles>C/C=C/C=O</smiles><smiles>CCOC(=O)CC</smiles><smiles>CCOC(=O)C=CC(=O)OCC</smiles><smiles>O=C(/C=C/c1ccccc1)c1ccccc1</smiles><smiles>O=C1C=CCCC1</smiles><smiles>O=C1C=CC(=O)C=C1</smiles><smiles>O=[N+]([O-])/C=C/c1ccccc1</smiles><smiles>C=Cc1ccc(C#N)cc1</smiles><smiles>C/C=C\c1c(F)c(F)c(F)c(F)c1F</smiles><smiles>C#Cc1ccc([N+](=O)[O-])cc1</smiles><smiles>O=S1(=O)C=CC=C1</smiles><smiles>CS(=O)(=O)CCCS</smiles><smiles>C=CS(=O)c1ccccc1</smiles><smiles>C=CC(=O)OCCO</smiles>

reacted to form hydrobrominated product $4 r$

2-Hydroxyethyl 3-bromopropanoate (compound number 4r): Prepared using the general procedure B from (2-hydroxyethyl acrylate 1r), tropylium tetrafluoroborate $\mathbf{2 b}$ and TMSBr $\mathbf{3}$ as anion source to give the title compound as a (151 mg, 77\% yield).

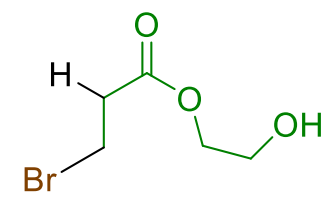

${ }^{1}$ H NMR (400 MHz, CDCl $) \delta 4.46(\mathrm{t}, J=6.2 \mathrm{~Hz}, 2 \mathrm{H}), 3.59(\mathrm{t}, J=6.8 \mathrm{~Hz}, 2 \mathrm{H}), 3.52(\mathrm{t}, J$ $=6.2 \mathrm{~Hz}, 2 \mathrm{H}), 2.97$ (t, $J=6.8 \mathrm{~Hz}, 2 \mathrm{H}) \mathrm{ppm}$;

${ }^{13}$ C NMR (100 MHz, CDCl $) \delta 170.2,64.3,37.7,28.5,25.7$ ppm.

IR (KBr) 2969, 1735, 1511,1384, $1345 \mathrm{~cm}^{-1}$;

HRMS (ESI+) m/z $[\mathrm{M}+\mathrm{Na}]^{+}$Calcd. for $\mathrm{C}_{5} \mathrm{H}_{9} \mathrm{Br}_{1} \mathrm{O}_{3}+\mathrm{Na} 218.9633$ (100\%), 220.9608 (97.3\%); Found 218.9629 (100\%), $220.9612(97.3 \%)$. 


\section{Characterization of products in Scheme 2}

5-(cyclohepta-2,4,6-trien-1-yl)furan-2(5H)-one (compound 4q) and 4-oxo-5phenylpentanoic acid ${ }^{3}$ (compound $\mathbf{4} \mathbf{q}^{\prime}$ ) Prepared using the general procedure B from $2(5 \mathrm{H})$ Furanone 1q, tropylium tetrafluoroborate $\mathbf{2 b}$ and $\mathrm{TMSBr} \mathbf{3}$ as anion source to give $\mathbf{4 q}$ as a colorless oil (104 mg, 60\% yield) and $\mathbf{4 q}$ ' as a white solid (74 mg, 38\% yield).

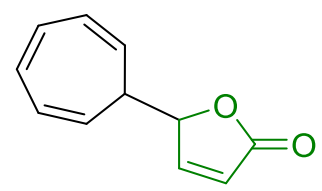

\section{$4 \mathbf{q}$}

${ }^{1}$ H NMR (400 MHz, CDCl 3$) \delta 7.55(\mathrm{dd}, J=5.7,1.8 \mathrm{~Hz}, 1 \mathrm{H}), 6.74-6.64(\mathrm{~m}, 2 \mathrm{H}), 6.36$ $6.27(\mathrm{~m}, 2 \mathrm{H}), 6.25(\mathrm{dd}, J=5.7,1.8 \mathrm{~Hz}, 1 \mathrm{H}), 5.37-5.24(\mathrm{~m}, 3 \mathrm{H}), 2.23-2.09$ (m, 1H) ppm.

${ }^{13}$ C NMR (100 MHz, $\left.\mathbf{C D C l}_{3}\right) \delta 172.9,154.9,131.4,131.1,126.8,126.3,122.8,120.2,119.9$, 82.9, 41.9 ppm.

IR (KBr): 3023, 2923, 1751, 1602, 1326, 1243, 1160, 1096, 894, 816, $752 \mathrm{~cm}^{-1}$;

HRMS (ESI+) m/z [M+Na]: calc. for $\mathrm{C}_{11} \mathrm{H}_{10} \mathrm{O}_{2}+\mathrm{Na}$ : 197.0578; found: 197.0572 .

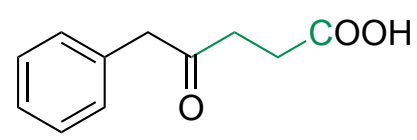

$4 q^{\prime}$

${ }^{1}$ H NMR (400 MHz, CDCl $)$ ) 7.41-7.20 (m, 5H), 3.76 (s, 2H), 2.81-2.74 (m, 2H), 2.65-2.58 $(\mathrm{m}, 2 \mathrm{H}) \mathrm{ppm}$. NMR data corresponded to that reported in literature. ${ }^{3}$

${ }^{13}$ C NMR (100 MHz, $\left.\mathbf{C D C l}_{3}\right) \delta 206.5,178.6,133.9,129.5,128.8,127.2,50.0,36.2,27.9$ ppm. NMR data corresponded to that reported in literature. ${ }^{3}$

A possible explanation for the formation of $\mathbf{4} \mathbf{q}$ and $\mathbf{4} \mathbf{q}^{\prime}$ is depicted below: 


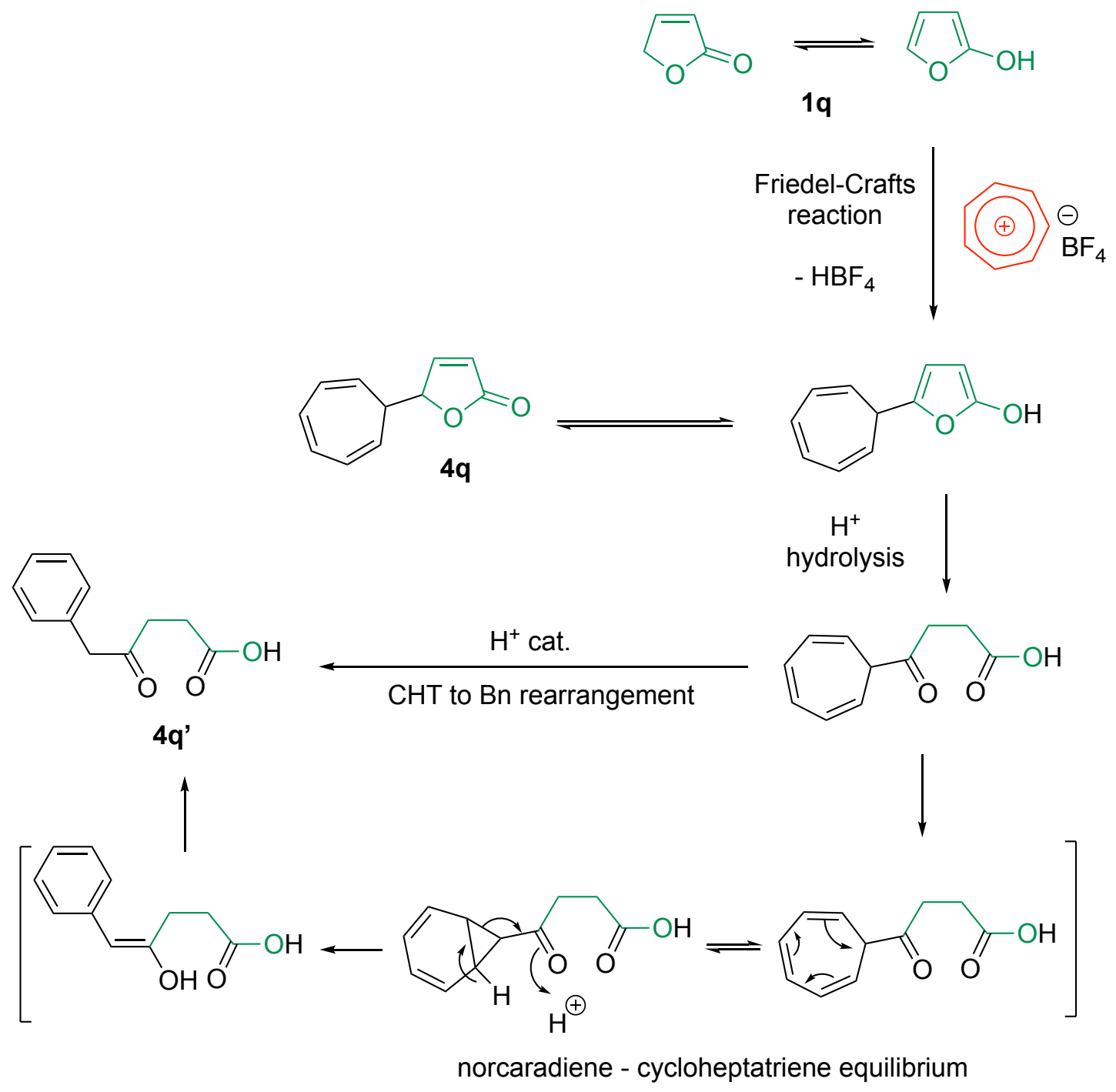

Hydrolysis of $\mathbf{4 q}$ led to the formation of $\mathbf{4 q}$ ' in quantitative yield. 


\section{Characterization of products in Scheme 3}

Methyl ( $E$ or $Z$ )-3-bromo-2-(cyclohepta-2,4,6-trien-1-yl)acrylate (compound number 6a): Prepared using the general procedure B from (methyl propiolate 5a), tropylium tetrafluoroborate $\mathbf{2 b}$ and TMSBr $\mathbf{3}$ as anion source to give an inseparable mixture of the $E$ and the $Z$-isomers as a colorless oil (244 mg, 96\% total yield, $E: Z$ (determined by ${ }^{1} \mathrm{H}$ NMR of the crude product $)=1: 4.5$ ). After several re-columning attempts, we were able to separate some fractions with enriched $Z$-isomer for ${ }^{1} \mathrm{H}$ and ${ }^{13} \mathrm{C}$ NMR analysis.

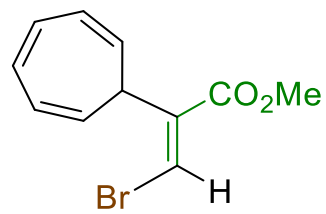

$Z$-isomer (contaminated with $\sim \mathbf{1 0} \%$ E-isomer): ${ }^{1}$ HNMR (400 MHz, $\left.\mathbf{C D C l}_{3}\right) \delta 6.68-6.60$ (m, 2H), $6.55(\mathrm{~d}, J=1.3 \mathrm{~Hz}, 1 \mathrm{H}), 6.29-6.19(\mathrm{~m}, 2 \mathrm{H}), 5.40-5.35(\mathrm{~m}, 2 \mathrm{H}), 3.81(\mathrm{~s}, 3 \mathrm{H}), 2.92$ (m, 1H) ppm;

${ }^{13}$ CNMR (100 MHz, $\left.\mathbf{C D C l}_{3}\right) \delta 164.9,138.6,131.1,125.8,124.7,124.6,124.4,122.5,110.7$, $52.5,44.0,41.5 \mathrm{ppm}$.

IR (KBr) 2870, 1720, 1635, 1522, $1406 \mathrm{~cm}^{-1}$;

HRMS (ESI+) $\mathbf{m} / \mathbf{z}:[\mathrm{M}+\mathrm{Na}]^{+}$Calcd. for $\mathrm{C}_{11} \mathrm{H}_{11} \mathrm{Br}_{1} \mathrm{O}_{2}+\mathrm{Na} 276.9840$ (100\%), 278.9820 (97.3\%); Found 276.9838 (100\%), 278.9814 (97.3\%).

Ethyl (Z)-3-bromo-2-(cyclohepta-2,4,6-trien-1-yl)acrylate (compound number 6b): Prepared using the general procedure B from (ethyl propiolate 5b), tropylium tetrafluoroborate $\mathbf{2 b}$ and TMSBr $\mathbf{3}$ as anion source to give an inseparable mixture of the $E$ and the $Z$-isomers as a colorless oil $\left(265 \mathrm{mg}, 99 \%\right.$ total yield, $E: Z$ (determined by ${ }^{1} \mathrm{H}$ NMR of the crude product $)=1: 4.2$ ). After several re-columning attempts, we were able to separate some fractions with enriched $Z$-isomer for ${ }^{1} \mathrm{H}$ and ${ }^{13} \mathrm{C}$ NMR analysis.

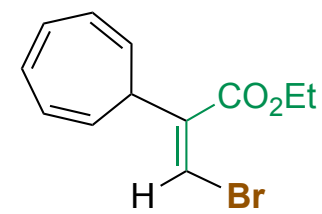

$Z$-isomer: ${ }^{1}$ H NMR (400 MHz, $\left.\mathbf{C D C l}_{3}\right) \delta 6.68-6.61(\mathrm{~m}, 2 \mathrm{H}), 6.51(\mathrm{~d}, J=1.3 \mathrm{~Hz}, 1 \mathrm{H}), 6.29$ - $6.19(\mathrm{~m}, 2 \mathrm{H}), 5.44-5.34(\mathrm{~m}, 2 \mathrm{H}), 4.31$ (q, $J=7.1 \mathrm{~Hz}, 2 \mathrm{H}), 2.89(\mathrm{~m}, 1 \mathrm{H}), 1.34$ (t, $J=7.1$ $\mathrm{Hz}, 3 \mathrm{H}) \mathrm{ppm}$ 
${ }^{13}$ C NMR (100 MHz, $\left.\mathbf{C D C l}_{3}\right) \delta 166.6,138.5,131.1,125.8,122.4,110.0,61.5,44.1,14.3$ ppm.

IR (KBr) 2980, 1716, 1604, 1463, $1393 \mathrm{~cm}^{-1}$;

HRMS (ESI+) $\mathbf{m} / \mathbf{z}:[\mathrm{M}+\mathrm{Na}]^{+}$Calcd. for $\mathrm{C}_{12} \mathrm{H}_{13} \mathrm{BrO}_{2}+\mathrm{Na} 290.9997$ (100\%), 292.9976 (97.3\%); Found 290.9993 (100\%), 292.9970 (97.3\%).

Dimethyl 2-bromo-3-(cyclohepta-2,4,6-trien-1-yl)but-2-enedioate (compound 6c) Prepared using the general procedure $B$ from dimethyl acetylenedicarboxylate 5c, tropylium tetrafluoroborate $\mathbf{2 b}$ and TMSBr $\mathbf{3}$ as anion source to give a mixture of the $E$ - and the $Z$ isomers as a colorless oil $\left(159 \mathrm{mg}, 51 \%\right.$ total yield, $E: Z$ (determined by ${ }^{1} \mathrm{H}$ NMR) $=1: 2$ ). After several re-columning attempts, we were able to separate some fractions with enriched Zor $E$-isomer for ${ }^{1} \mathrm{H}$ and ${ }^{13} \mathrm{C}$ NMR analysis.

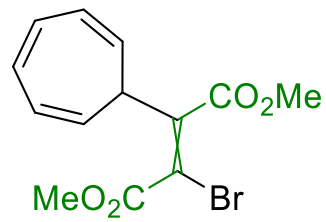

E-isomer: ${ }^{1} \mathbf{H}$ NMR (400 MHz, $\left.\mathbf{C D C l}_{3}\right) \delta 6.74-6.67(\mathrm{~m}, 2 \mathrm{H}), 6.28-6.19(\mathrm{~m}, 2 \mathrm{H}), 5.37$ $5.27(\mathrm{~m}, 2 \mathrm{H}), 3.92(\mathrm{~s}, 3 \mathrm{H}), 3.78(\mathrm{~s}, 3 \mathrm{H}), 3.16(\mathrm{tt}, J=5.5,1.4 \mathrm{~Hz}, 1 \mathrm{H}) \mathrm{ppm}$;

${ }^{13}$ C NMR (100 MHz, $\left.\mathbf{C D C l}_{3}\right) \delta 166.9,163.1,146.2,131.1,125.0,119.9,112.2,53.3,52.6$, $42.3 \mathrm{ppm}$;

$Z$-isomer: ${ }^{1} \mathbf{H}$ NMR (400 MHz, $\left.\mathbf{C D C l}_{3}\right) \delta 6.76$ - 6.69 (m, 2H), 6.33 - 6.24 (m, 2H), 5.29 $5.20(\mathrm{~m}, 2 \mathrm{H}), 3.90(\mathrm{~s}, 3 \mathrm{H}), 3.88(\mathrm{~s}, 3 \mathrm{H}), 2.98(\mathrm{tt}, J=5.4,1.5 \mathrm{~Hz}, 1 \mathrm{H}) \mathrm{ppm}$;

${ }^{13}$ C NMR (100 MHz, $\left.\mathbf{C D C l}_{3}\right) \delta 166.6,163.2,145.9,131.0,125.5,119.0,118.8,53.7,52.9$, $44.5 \mathrm{ppm}$;

IR (KBr) 3015, 2956, 1725, 1609, 1425, $1252 \mathrm{~cm}^{-1}$;

HRMS (ESI+) m/z: Calc. for $\mathrm{C}_{13} \mathrm{H}_{13} \mathrm{BrO}_{4}+\mathrm{Na}: 334.9895$ (100\%), 336.9874 (97.3\%); Found 334.9891 (100\%), $336.9869(97.3 \%)$. 


\section{Characterization data of products in Scheme 4}

Methyl 2-(cyclohepta-2,4,6-trien-1-yl)acrylate (compound 12): Prepared using the general procedure A from methyl acrylate $\mathbf{1 b}$ and tropylium bromide $\mathbf{2 a}$, followed by quenching with aq. $\mathrm{NH}_{3}$ and stirring for another $24 \mathrm{~h}$ to give the title compound as a colorless oil $(113 \mathrm{mg}$, $64 \%$ yield over two steps, or 95\% yield for dehydrobromination step).

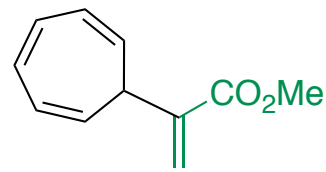

${ }^{1}$ H NMR (400 MHz, CDCl$\left._{3}\right) \delta 6.68-6.61(\mathrm{~m}, 2 \mathrm{H}), 6.34(\mathrm{~s}, 1 \mathrm{H}), 6.27-6.18(\mathrm{~m}, 2 \mathrm{H}), 5.76$ (s, 1H), $5.37-5.28(\mathrm{~m}, 2 \mathrm{H}), 3.77(\mathrm{~s}, 3 \mathrm{H}), 2.90(\mathrm{td}, J=6.1,1.2 \mathrm{~Hz}, 1 \mathrm{H}) \mathrm{ppm}$.

${ }^{13}$ C NMR (100 MHz, $\left.\mathbf{C D C l}_{3}\right) \delta 167.3,140.4,130.8,125.1,124.9,124.5,52.0,41.0$ ppm.

IR (KBr) 2878, 1726, 1637, 1520, $1412 \mathrm{~cm}^{-1}$;

HRMS (ESI+) m/z [M+Na]: Calc. for $\mathrm{C}_{11} \mathrm{H}_{12} \mathrm{O}_{2}+\mathrm{Na}$ 199.0730; Found: 199.0730.

\section{Methyl-3-bromo-2-(1,3-dioxodecahydro-4,6-ethanocyclopropa[f]isoindol-5-}

yl)propanoate (compound number 13$)$ : To a solution of $\mathbf{4 a}(0.5 \mathrm{mmol})$ in DCE $(5 \mathrm{~mL})$ was added maleimide $(0.5 \mathrm{mmol}, 1$ equiv.). After stirring for $24 \mathrm{~h}$ at room temperature, the reaction mixture was concentrated under reduced pressure. The residue was purified by flash column chromatography (silica-gel, hexanes/EtOAc) to obtain the title compound as a bright white crystals (166 mg, 94\% yield). X-ray crystal structure revealed that it is a racemic mixture of the two enantiomers below. See page S30 for more details.
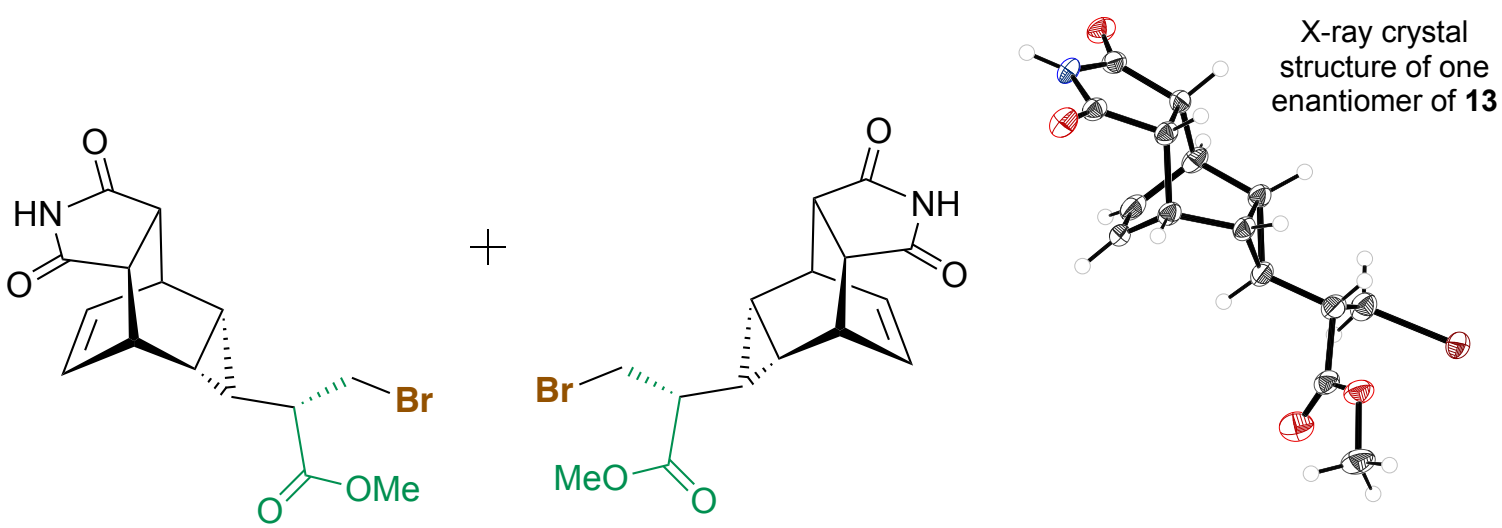

${ }^{1}$ H NMR (400 MHz, CDCl 3 ) $\delta 7.96$ (bs, 1H), 5.85 (dd, $\left.J=4.8,3.6 \mathrm{~Hz}, 2 \mathrm{H}\right), 3.74$ (s, 3H), $3.56(\mathrm{t}, J=9.8,1 \mathrm{H}), 3.48-3.41(\mathrm{~m}, 3 \mathrm{H}), 2.96(\mathrm{dd}, J=1.6,1.8 \mathrm{~Hz}, 2 \mathrm{H}), 2.28-2.23(\mathrm{~m}, 1 \mathrm{H})$, $1.22(\mathrm{~m}, 1 \mathrm{H}), 1.07(\mathrm{~m}, 1 \mathrm{H}), 0.82(\mathrm{dt}, J=9.8,3.0 \mathrm{~Hz}, 1 \mathrm{H}) \mathrm{ppm}$; 
${ }^{13}$ C NMR (100 MHz, $\left.\mathbf{C D C l}_{3}\right) \delta 178.1,178.0,172.6,129.0,128.5,52.4,50.1,32.9,32.9$, $31.4,19.9,17.4,16.0$ ppm.

IR (KBr) 3017, 2950, 2851, 1718, 1628, 1435, $1406 \mathrm{~cm}^{-1}$;

HRMS (ESI+) m/z: $[\mathrm{M}+\mathrm{Na}]^{+}$Calcd. for $\mathrm{C}_{15} \mathrm{H}_{18} \mathrm{Br}_{1} \mathrm{NO}_{4}+\mathrm{Na} 376.0160$ (100\%), 378.0135 (97.3\%); Found 376.0157 (100\%), 378.0132 (97.3\%).

Methyl 2-bromo-2-phenyl-3-hydroxypropanoate (compound 14): To a solution of 4a (0.5 $\mathrm{mmol})$ in $\mathrm{MeCN}(5 \mathrm{~mL})$ was added nitrosonium tetrafluoroborate (1.0 mmol, 2 equiv.). After stirring for $1 \mathrm{~h}$ at room temperature, hydrogen peroxide (30\% w/w aq. solution, 5 equiv.) was added and the reaction mixture was stirred for another $12 \mathrm{~h}$. The reaction was quenched by $10 \% \mathrm{w} / \mathrm{w}$ aq. sodium metabisulfite solution. The organic products were extracted by DCM (2 x $15 \mathrm{~mL}$ ), dried over sodium sulfate and concentrated under reduced pressure. The residue was purified by flash column chromatography (silica-gel, hexanes/EtOAc) to obtain the title compound as a colorless oil ( $83 \mathrm{mg}, 63 \%$ yield). It should be noted that, apart from the oxidative ring-contraction, the reaction conditions also led to the oxidation of the side-chain and the rearrangement of the bromo functional group.

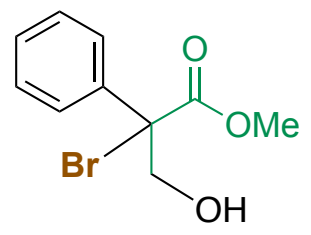

${ }^{1}$ H NMR (400 MHz, CDCl $\left.{ }_{3}\right) \delta$ 7.50-7.47 (m, 2H), 7.39-7.32 (m, 3H), 4.19 (s, 2H), 3.86 (s, 3H) ppm;

${ }^{13}$ C NMR (100 MHz, CDCl $) \delta 170.0,136.7,129.1,128.7,127.6,70.2,67.9,53.8$ ppm.

IR (KBr) 3439, 2951, 1723, 1494, $1444 \mathrm{~cm}^{-1}$;

HRMS (ESI+) m/z: $[\mathrm{M}+\mathrm{Na}]^{+}$Calcd. for $\mathrm{C}_{10} \mathrm{H}_{11} \mathrm{Br}_{1} \mathrm{O}_{3}+\mathrm{Na} 280.9789$ (100\%), 282.9769

(97.3\%); Found 280.9785 (100\%), 282.9763 (97.3\%)

Methyl 2-(bromomethyl)-3-chloro-3-phenyl-3-propanoate (compound 15): Compound 4a $(0.5 \mathrm{mmol})$ was dissolved by $5 \mathrm{~mL}$ of DCM in a $21 \mathrm{~mL}$ vial charged a stirrer bar. The solution was cooled down to $0{ }^{\circ} \mathrm{C}$ and $\mathrm{TeCl}_{4}$ was added in portion. The reaction mixture was warmed up to room temperature and stirred overnight. After completion, the mixture was filtered through a plug of celite, washed by $15 \mathrm{~mL}$ of DCM and concentrated under reduced pressure. The residue was purified by flash column chromatography (silica-gel, hexanes/EtOAc) to 
obtain an inseparable mixture of two diastereoisomers (1:1.1, ratio by NMR analysis) as a colorless oil (119 mg, 82\%).<smiles>COC(=O)C(CBr)C(Cl)c1ccccc1</smiles>

${ }^{1} \mathbf{H}$ NMR (300 MHz, CDCl$) \delta 7.47-7.31(\mathrm{~m}, 10.5 \mathrm{H}), 5.09(\mathrm{~d}, J=10.1 \mathrm{~Hz}, 1 \mathrm{H}), 5.08(\mathrm{~d}, J=$ $9.8 \mathrm{~Hz}, 1.1 \mathrm{H}), 3.97-3.70(\mathrm{~m}, 6.4 \mathrm{H}), 3.58-3.43(\mathrm{~m}, 4.9 \mathrm{H}), 3.32-3.12(\mathrm{~m}, 2.1 \mathrm{H}) \mathrm{ppm}$.

${ }^{13} \mathbf{C}$ NMR (75 MHz, $\left.\mathbf{C D C l}_{3}\right) \delta 170.9,170.1,138.5,137.6,129.4,129.1,129.0,128.7,128.6$, $127.5,127.4,62.3,61.0,56.5,56.4,52.5,52.2,30.3,29.3 \mathrm{ppm}$.

IR (KBr): 3034, 2324, 1739, 1437, $1347 \mathrm{~cm}^{-1}$;

HRMS (ESI+): $\mathrm{m} / \mathrm{z}$ [M+Na] Calc. for $\mathrm{C}_{11} \mathrm{H}_{12} \mathrm{Br}_{1} \mathrm{Cl}_{1} \mathrm{O}_{2}+\mathrm{Na} 312.9607$ (100\%), 314.9586; (97.3\%); Found: 312.9604 (100\%), 314.9579 (97.3\%).

\section{References}

[1] W. Von E.Doering, L. H. Knox, J. Am. Chem. Soc. 1954, 76, 3203-3206

[2] X. Li, S. M. Singh, F. Labrie, Synth. Commun. 1994, 24, 733-743.

[3] A. C. Boye, D. Meyer, C. K. Ingison, A. N. French, T. Wirth, Org. Lett. 2003, 5, $2157-$ 2159. 


\section{Computational Studies}

Density functional theory (M06-2X/6-31+G(d,p) and SMD) calculations were carried out to investigate the mechanism of the reaction. The calculations indicate that tropylium bromide exists as an ion-pair in dichloroethane solution $\left(-21.8 \mathrm{~kJ} \mathrm{~mol}^{-1}\right.$ relative to separate ions). This ion-pair may in turn mediate the bromocycloheptrienylation of 1a through two possible pathways (a) $\mathrm{CO}$ addition to tropylium followed by addition of bromide to form intermediate 10, which can undergo sigmatropic rearrangement (red arrows in structure 10) to form the product 4a. (b) direct addition of tropylium and bromide to the olefin to form $4 \mathbf{a}$.

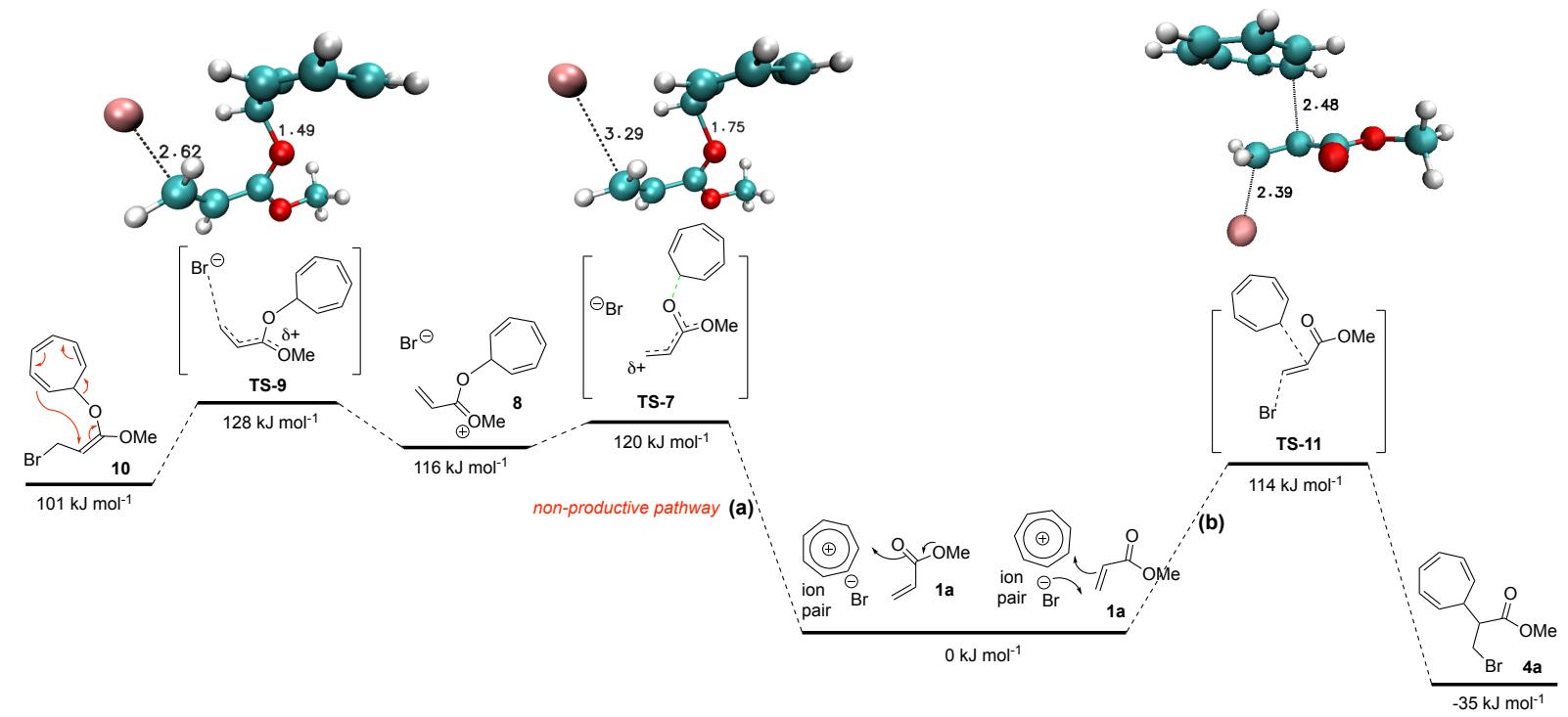

Figure S2. M06-2X/6-31+G(d,p) + SMD (dichloroethane) free energy profile.

As shown in Figure S2, the first two stes of pathway (a) are associated with a relatively high Gibbs free energy barrier (TS-7 and TS-9) of 120 and $128 \mathrm{~kJ} \mathrm{~mol}^{-1}$ respectively. Inspection of the geometries of TS-7 and $\mathbf{8}$ indicates that there is a significant shortening ( $c a .0 .04 \AA)$ of both the $\mathrm{C}-\mathrm{OMe}$ and $\mathrm{C}-\mathrm{C}(\mathrm{O})$ bonds relative to $\mathbf{1 a}$; the structure of intermediate $\mathbf{8}$ depicted in Figure S2 represents one of the resonance forms. Attempts to locate the transition state for the sigmatropic rearrangement of $\mathbf{1 0}$ to form $\mathbf{4 a}$ were unsuccessful and pathway (a) was deemed an unproductive pathway. On the other hand, the direct addition of tropylium and bromide across the olefin double bond is associated with a significantly lower barrier of $114 \mathrm{~kJ} \mathrm{~mol}^{-1}$ to transition state TS-11. This pathway (b) leads directly to product $\mathbf{4 a}$, which is predicted to be an exergonic process $\left(\Delta \mathrm{G}_{\mathrm{rxn}}-35 \mathrm{~kJ} \mathrm{~mol}^{-1}\right)$. 


\section{Computational Details}

All electronic structure calculations were carried using the Gaussian16 program. ${ }^{1}$ Geometries and harmonic frequency calculations were performed at the M06-2X/6-31+G(d,p) ${ }^{2}$ level directly within the SMD implicit solvent model $^{3}$ (DCE solvent). ${ }^{4}$ Systematic conformer searches were carried out to locate the lowest energy conformers of each species in Figure S2. Frequency calculations confirm that all reactant and intermediates are true minimum energy structures and that all transition states are true first-order saddle points. Intrinsic reaction coordinate $^{5}$ calculations were also carried out to ascertain that the transition states connected the correct reactants and products. Bond order calculations were carried out using the Wiberg scheme. ${ }^{6}$ Standard state corrections were applied so that all reported solution phase free energies correspond to a standard state of $1 \mathrm{~mol} \mathrm{~L}^{-1}$.

\section{Cartesian coordinates of M06-2X/6-31+G(d,p)/SMD(dichloroethane) optimized geometries.}

$1 \mathbf{a}$

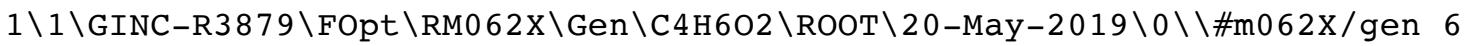
D OPT Freq=Noraman INT(grid=ultrafine) SCRF=(SMD, Solvent=dichloroethan e) $\backslash \backslash$ vinylester.smd $\backslash \backslash 0,1 \backslash 0,-0.2763531994,-0.1645432708,0 . \backslash C,-1.61005949$ $32,-0.2787681622,0 . \backslash \mathrm{C},-2.0296560912,-1.7033415094,0 . \backslash \mathrm{C}, 0.2384104397,1$. $1731344304,0 . \backslash 0,-2.359623973,0.676651045,0 . \backslash \mathrm{C},-3.3220285399,-2.0267404$ $97,0 . \backslash \mathrm{H},-1.2407174465,-2.4488008199,0 . \backslash \mathrm{H}, 1.322362504,1.0716504767,0 . \backslash \mathrm{H}$ $,-0.0944498845,1.7063971995,0.893057012 \backslash \mathrm{H},-0.0944498845,1.7063971995,-$ $0.893057012 \backslash \mathrm{H},-4.0879561691,-1.2555786925,0 . \backslash \mathrm{H},-3.6418782538,-3.063857$ $4072,0 . \backslash \backslash$ Version=ES64L-G16RevA. 03 $\backslash$ State $^{2} 1-\mathrm{A}^{\prime} \backslash \mathrm{HF}=-306.3566243 \backslash \mathrm{RMSD}=5.45$ $2 e-09 \backslash \mathrm{RMSF}=4.235 \mathrm{e}-05 \backslash \mathrm{Dipole}=0.5558433,-0.5443501,0 . \backslash$ Quadrupole $=1.04302$ $66,1.487114,-2.5301406,5.8974151,0 ., 0 . \backslash P G=C S[S G(C 4 H 4 O 2), x(H 2)] \backslash \backslash @$

\section{Tropylium cation}

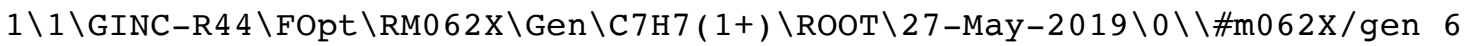
D INT(grid=ultrafine) OPT Freq=noraman SCRF=(SMD, Solvent=dichloroethan e) \\trop-cation.freq $\backslash \backslash 1,1 \backslash C,-2.7912842413,1.7249249771,1.1663661029 \backslash \mathrm{C}$, $-4.1765511529,1.6346978294,1.027237556 \backslash C,-5.1566950487,2.5038934335,1$. $5066988138 \backslash \mathrm{C},-4.993301823,3.676901047,2.2439602116 \backslash \mathrm{C},-3.8103097204,4.2$ $708655692,2.6845701815 \backslash \mathrm{C},-2.4972589542,3.8380648046,2.4961955533 \backslash \mathrm{C},-2$. $0439574822,2.7048105926,1.8205236546 \backslash \mathrm{H},-4.5397275231,0.7743713351,0.47$ $21680423 \backslash \mathrm{H},-6.1827707924,2.2313832525,1.2755373285 \backslash \mathrm{H},-5.9093477486,4.1$ $963390368,2.511079105 \backslash \mathrm{H},-3.9266023888,5.1916777931,3.2494306856 \backslash \mathrm{H},-1.7$ $262677119,4.4664132952,2.933371635 \backslash \mathrm{H},-0.9664050526,2.5675125848,1.8012$ $185019 \backslash \mathrm{H},-2.21839036,0.925184449,0.704472628 \backslash \backslash$ Version=ES64L-G16RevA 03 $\backslash$ State $=1-\mathrm{A} \backslash \mathrm{HF}=-270.6366223 \backslash \mathrm{RMSD}=4.750 \mathrm{e}-09 \backslash \mathrm{RMSF}=1.211 \mathrm{e}-04 \backslash \mathrm{Dipole}=-0.000$ $4847,-0.001364,-0.0002266 \backslash Q u a d r u p o l e=4.9150984,0.8336515,-5.7487498,-0$ $.3985817,0.6526003,6.6436249 \backslash \mathrm{PG}=\mathrm{C} 01[\mathrm{X}(\mathrm{C} 7 \mathrm{H} 7)] \backslash \backslash @$

\section{Tropylium-bromide ion pair}

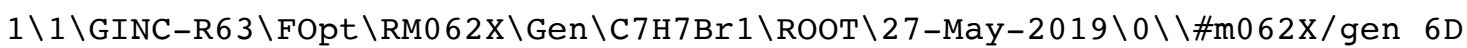
INT (grid=ultrafine) OPT Freq=noraman SCRF=(SMD, Solvent=dichloroethane 
)$\backslash \backslash$ trop-bromide. freq $\backslash \backslash 0,1 \backslash \mathrm{Br},-4.6901813437,0.0596262954,-2.4005751586 \backslash$ $C,-4.4476605393,-1.1127215815,0.98000394 \backslash C,-4.6647316654,-0.9875301006$ $, 2.3520398849 \backslash \mathrm{C},-4.311383943,-1.8733146113,3.3704142667 \backslash \mathrm{C},-3.655473762$ $2,-3.1002713955,3.2661648683 \backslash C,-3.1899492703,-3.7469200834,2.120907326$ $6 \backslash \mathrm{C},-3.2653246946,-3.32475065,0.7932537051 \backslash \mathrm{C},-3.8251875938,-2.15199654$ $66,0.2868356031 \backslash \mathrm{H},-4.8022301063,-0.3038992585,0.34169136 \backslash \mathrm{H},-5.17544404$ $46,-0.0823638402,2.6697649236 \backslash \mathrm{H},-4.5823698651,-1.5684057617,4.37775932$ $38 \backslash \mathrm{H},-3.4841051266,-3.6228329382,4.2035417592 \backslash \mathrm{H},-2.7048651151,-4.70539$ $77121,2.2857164584 \backslash \mathrm{H},-2.8310992873,-3.9976055844,0.0585324022 \backslash \mathrm{H},-3.784$ $113643,-2.0033862314,-0.7918606633 \backslash \backslash$ Version=ES64L-G16RevA.03 $\backslash$ State $=1-A$ $\backslash \mathrm{HF}=-2844.7036191 \backslash \mathrm{RMSD}=6.852 \mathrm{e}-09 \backslash \mathrm{RMSF}=4.724 \mathrm{e}-06 \backslash \mathrm{Dipole}=1.3979445,-4.27$ $06084,7.6581651 \backslash$ Quadrupole $=-1.9488805,3.180028,-1.2311474,-4.7839539$,$3.1812431,5.3092961 \backslash \mathrm{PG}=\mathrm{C} 01[\mathrm{X}(\mathrm{C} 7 \mathrm{H} 7 \mathrm{Br} 1)] \backslash \backslash @$

\section{TS-7}

$1 \backslash 1 \backslash G I N C-R 4152 \backslash F T S \backslash R M 062 X \backslash G e n \backslash C 11 \mathrm{H} 13 B r 102 \backslash R O O T \backslash 23-M a y-2019 \backslash 0 \backslash \backslash \#$ m062x/g en 6D INT(grid=ultrafine) OPT=(TS, calcfC, noeigentest) Freq=noraman SCR $\mathrm{F}=($ SMD, Solvent $=d i c h l o r o e t h a n e) \backslash \backslash T S$. freq $\backslash \backslash 0,1 \backslash 0,1.949351828,2.844258564$ $9,0.0689979838 \backslash \mathrm{C}, 1.0032840934,1.9653798941,-0.128009672 \backslash \mathrm{C},-0.328612177$ $8,2.5418456352,-0.2686596434 \backslash \mathrm{C}, 3.3021221204,2.3637951704,0.2422976672 \backslash$ $0,1.308255938,0.7474956414,-0.2006290739 \backslash C,-1.2719473919,1.9392953742$, $-1.0027519925 \backslash \mathrm{H},-0.4762454156,3.5137986061,0.1895095614 \backslash \mathrm{H}, 3.8909971947$ , $3.2542840117,0.4489087458 \backslash \mathrm{H}, 3.6396921856,1.8788363249,-0.674483381 \backslash \mathrm{H}$, $3.3406442583,1.6668844584,1.0805763824 \backslash \mathrm{Br},-3.304604156,-0.3138239393,0$ $.2565715024 \backslash \mathrm{H},-2.2431666206,2.3987431719,-1.1447475626 \backslash \mathrm{H},-1.0944411119$ $, 1.0026103906,-1.5210036723 \backslash \mathrm{C}, 0.2681717243,-0.5618746268,0.3230000728 \backslash$ $C, 1.0127154466,-1.0989070345,1.450103357 \backslash C, 2.1444337262,-1.8517715137$, $1.3855074691 \backslash \mathrm{C}, 2.8130414595,-2.3528068278,0.2245572154 \backslash \mathrm{C}, 2.2910723715$, $-2.5030777151,-1.0287969519 \backslash \mathrm{C}, 0.958609283,-2.1990257699,-1.452824782 \backslash \mathrm{C}$ $, 0.0478639177,-1.3865876281,-0.8532917806 \backslash \mathrm{H},-0.623738439,-0.0065441263$ $, 0.6112458895 \backslash \mathrm{H}, 0.6488328356,-0.8075612651,2.4319060674 \backslash \mathrm{H}, 2.6211894404$ $,-2.0862237039,2.3348106382 \backslash \mathrm{H}, 3.8151297991,-2.7405714766,0.3885119284 \backslash$ $\mathrm{H}, 2.918713374,-2.9985413946,-1.76513582 \backslash \mathrm{H}, 0.6462595584,-2.6706501764$,$2.3819869989 \backslash \mathrm{H},-0.9434342418,-1.2938010457,-1.2922191497 \backslash \backslash$ Version=ES6 4 L-G16RevA.03 \State $=1-A \backslash H F=-3151.0335691 \backslash$ RMSD $=4.118 e-09 \backslash$ RMSF $=2.382 e-06 \backslash$ Dipole $=7.2187234,1.01412,-0.5063549 \backslash$ Quadrupole $=-17.1287233,11.0596342$, $6.0690891,-7.2576479,7.1357264,2.6415257 \backslash \mathrm{PG}=\mathrm{C} 01[\mathrm{X}(\mathrm{C} 11 \mathrm{H} 13 \mathrm{Br} 1 \mathrm{O} 2)] \backslash \backslash @$

8

$1 \backslash 1 \backslash G I N C-R 4097 \backslash F O p t \backslash R M 062 X \backslash G e n \backslash C 11 \mathrm{H} 13 \mathrm{Br} 102 \backslash R O O T \backslash 25-M a y-2019 \backslash 0 \backslash \backslash \#$ 0 62X/ gen 6D INT(grid=ultrafine) OPT Freq=noraman SCRF=(SMD, Solvent=dichloro

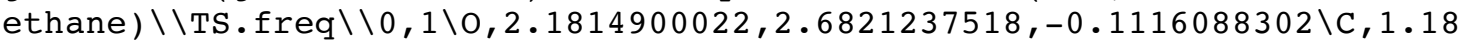
$02629124,1.8643958513,-0.1558892424 \backslash \mathrm{C},-0.1131364485,2.4735449431,-0.38$ $4192609 \backslash C, 3.5099268875,2.1569477753,0.1483321975 \backslash 0,1.4522884709,0.6257$ $328023,-0.0057056987 \backslash \mathrm{C},-1.0946043652,1.8530163291,-1.0569003988 \backslash \mathrm{H},-0.1$ $938869598,3.5061695462,-0.0626859696 \backslash \mathrm{H}, 4.1486183216,3.0352855801,0.190$ $9118906 \backslash \mathrm{H}, 3.803542788,1.4970815523,-0.6687062016 \backslash \mathrm{H}, 3.5091518845,1.6222$ $712995,1.0986841644 \backslash \mathrm{Br},-3.1407693392,-0.0618125724,0.4477516918 \backslash \mathrm{H},-2.0$ $192773031,2.3746745783,-1.2743434342 \backslash \mathrm{H},-0.9909004656,0.8563585632,-1.4$ $697516352 \backslash \mathrm{C}, 0.4449608175,-0.4394784649,0.3711926682 \backslash \mathrm{C}, 1.0570680174,-1$. $1917477056,1.5005742763 \backslash \mathrm{C}, 2.2387720318,-1.8430035095,1.4123254455 \backslash \mathrm{C}, 3$. $0233838771,-2.0595101227,0.2213030433 \backslash \mathrm{C}, 2.5482126364,-2.1261102596,-1$. $0532955903 \backslash \mathrm{C}, 1.1679607956,-1.997067108,-1.4524442938 \backslash \mathrm{C}, 0.1955829522,-1$ $.3178689265,-0.803701064 \backslash \mathrm{H},-0.4698678933,0.0698593603,0.6779956493 \backslash \mathrm{H}, 0$ $.5251508819,-1.1608957492,2.4473904774 \backslash \mathrm{H}, 2.650244845,-2.253696213,2.33$ $2121118 \backslash \mathrm{H}, 4.0690366992,-2.3142559815,0.3768802589 \backslash \mathrm{H}, 3.2459224579,-2.42$ $97445208,-1.8300281457 \backslash \mathrm{H}, 0.8879951578,-2.5140286539,-2.3681570945 \backslash \mathrm{H},-0$ $.837637662,-1.3735121451,-1.1402156732 \backslash \backslash$ Version=ES64L-G16RevA.03\State $=1-\mathrm{A} \backslash \mathrm{HF}=-3151.0368203 \backslash \mathrm{RMSD}=6.296 \mathrm{e}-09 \backslash \mathrm{RMSF}=7.099 \mathrm{e}-06 \backslash \mathrm{Dipole}=6.9085499,1$ $.5859943,-0.9573561 \backslash$ Quadrupole $=-15.6381014,10.0006615,5.6374399,-2.019$ $8341,7.6245907,0.7036331 \backslash \mathrm{PG}=\mathrm{C} 01[\mathrm{X}(\mathrm{C} 11 \mathrm{H} 13 \mathrm{Br} 1 \mathrm{O} 2)] \backslash \backslash @$ 


\section{TS-9}

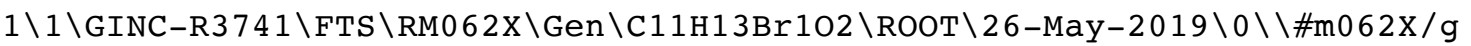
en 6D INT(grid=ultrafine) OPT=(TS,calcfC, noeigentest) Freq=noraman SCR $\mathrm{F}=(\mathrm{SMD}$, Solvent=dichloroethane $) \backslash \backslash \mathrm{TS}$. freq $\backslash \backslash 0,1 \backslash 0,2.1079399872,2.69661501$ $07,-0.0144011643 \backslash \mathrm{C}, 1.1202691548,1.854988061,-0.1833179079 \backslash \mathrm{C},-0.1408328$ $725,2.4150060499,-0.4770078479 \backslash \mathrm{C}, 3.4079122102,2.1878165693,0.359029937$ $6 \backslash 0,1.4482853422,0.602021628,-0.099530944 \backslash \mathrm{C},-1.1520091387,1.7262114479$ $,-1.0999520833 \backslash \mathrm{H},-0.2350582893,3.4744911438,-0.2701403628 \backslash \mathrm{H}, 4.01994725$ $32,3.0747565088,0.5057176494 \backslash \mathrm{H}, 3.8098318921,1.569522557,-0.444610085 \backslash \mathrm{H}$ , 3.3311504985,1.6122038539,1.2825131653\Br,-2.9504942119,0.6770430808, $0.4931419037 \backslash \mathrm{H},-1.9848594288,2.2836210338,-1.5075695423 \backslash \mathrm{H},-0.995406123$ $5,0.7344999642,-1.5075153229 \backslash \mathrm{C}, 0.5014011223,-0.4495293519,0.3713523198$ $\backslash \mathrm{C}, 1.1872303004,-1.1710618572,1.4836487954 \backslash \mathrm{C}, 2.3624186217,-1.823220997$ $8,1.3388861496 \backslash \mathrm{C}, 3.065143106,-2.0783975486,0.1045440704 \backslash \mathrm{C}, 2.503495424$, $-2.1911445345,-1.1306176256 \backslash \mathrm{C}, 1.0973023741,-2.0821874051,-1.4357139986$ $\backslash \mathrm{C}, 0.1707562977,-1.381782974,-0.7444726472 \backslash \mathrm{H},-0.3948851501,0.057534168$ $, 0.7301624404 \backslash \mathrm{H}, 0.719869667,-1.1132496855,2.4629225126 \backslash \mathrm{H}, 2.8356116698$, $-2.2048347639,2.2415927948 \backslash \mathrm{H}, 4.120841167,-2.3227861662,0.1962200782 \backslash \mathrm{H}$, $3.1474946196,-2.5181045862,-1.9435631378 \backslash \mathrm{H}, 0.7563881824,-2.6356000876$, $-2.3084859379 \backslash \mathrm{H},-0.8802526752,-1.4596991188,-1.0149942096 \backslash \backslash$ Version=ES 6 $4 \mathrm{~L}-\mathrm{G} 16 \mathrm{RevA} .03 \backslash$ State $=1-\mathrm{A} \backslash \mathrm{HF}=-3151.0344978 \backslash \mathrm{RMSD}=2.998 \mathrm{e}-09 \backslash \mathrm{RMSF}=2.912 \mathrm{e}-06$ $\backslash$ Dipole $=5.0268734,0.0383932,-0.8119226 \backslash$ Quadrupole $=-9.8084262,5.0796933$ $, 4.7287329,1.427347,7.9403961,0.4256772 \backslash \mathrm{PG}=\mathrm{C} 01$ [X(C11H13Br1O2)] \\

\section{0}

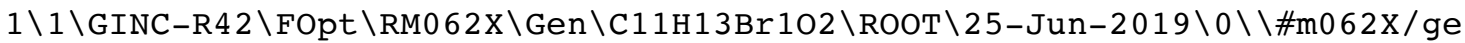
n 6D OPT Freq=Noraman INT(grid=ultrafine) SCRF=(SMD, Solvent=dichloroet hane $) \backslash \backslash$ trop-bromo-vinylester-axial.smd $\backslash \backslash 0,1 \backslash 0,-1.0515554882,0.23866619$ $53,-0.7178599161 \backslash \mathrm{C},-1.9672495428,0.0575007746,0.2656379508 \backslash \mathrm{C},-2.813779$ $2986,-0.9817613403,0.2473972596 \backslash \mathrm{C}, 0.3089716957,0.2373321877,-0.2604211$ $902 \backslash 0,-1.9731217115,0.9804601719,1.254624376 \backslash \mathrm{C},-3.9059538878,-1.116740$ $1656,1.2283639339 \backslash \mathrm{H},-2.6700580831,-1.7448360986,-0.509646256 \backslash \mathrm{H}, 0.48214$ $01252,1.0186341792,0.4851153445 \backslash \mathrm{H}, 0.9256666059,0.4231114248,-1.1393443$ $964 \backslash \mathrm{H}, 0.5532056882,-0.7400697915,0.168580954 \backslash \mathrm{H},-4.090844431,-0.1958790$ $616,1.7766962643 \backslash \mathrm{H},-4.8247807597,-1.5058212389,0.7940724316 \backslash \mathrm{Br},-3.4687$ $80055,-2.4604919949,2.6536896372 \backslash \mathrm{C},-1.9762667318,2.3718421077,0.806459$ $815 \backslash \mathrm{C},-3.1869613569,2.6759668894,-0.0250820775 \backslash \mathrm{C},-4.4585721114,2.56840$ $81563,0.4199756542 \backslash \mathrm{C},-4.8892154879,2.3314350973,1.7786742372 \backslash \mathrm{C},-4.1966$ $025411,2.6408822815,2.907709226 \backslash \mathrm{C},-2.8980128113,3.2708443053,2.9672046$ $459 \backslash \mathrm{C},-1.9300617122,3.2369470131,2.0258468809 \backslash \mathrm{H},-3.0147426204,2.941932$ $0884,-1.0653424935 \backslash \mathrm{H},-5.2549323624,2.6866182148,-0.3128466419 \backslash \mathrm{H},-5.910$ $1238893,1.9763092368,1.9020637413 \backslash \mathrm{H},-4.7100793721,2.513255796,3.858005$ $6502 \backslash \mathrm{H},-2.6944540577,3.8437422617,3.8701461386 \backslash \mathrm{H},-1.0338186325,3.83460$ $43961,2.1703252079 \backslash \mathrm{H},-1.0777271705,2.5372669133,0.2083336226 \backslash \backslash$ Version= ES6 4L-G16RevA.03 \State $=1-A \backslash H F=-3151.0454685 \backslash$ RMSD $=6.137 \mathrm{e}-09 \backslash \mathrm{RMSF}=4.219 \mathrm{e}$ $-06 \backslash \mathrm{Dipole}=0.4258721,1.6934547,-0.7687591 \backslash$ Quadrupole $=8.8203395,-6.2156$ $58,-2.6046815,1.4249943,-1.6284664,4.2938633 \backslash \mathrm{PG}=\mathrm{C} 01 \quad[\mathrm{X}(\mathrm{C} 11 \mathrm{H} 13 \mathrm{Br} 1 \mathrm{O} 2)] \backslash \backslash$ (a

\section{TS-11}

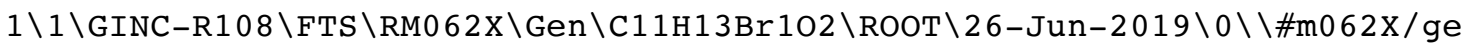
$\mathrm{n}$ 6D INT(grid=ultrafine) OPT=(TS,calcfc, noeigentest) Freq=noraman SCRF $=($ SMD, Solvent $=$ dichloroethane $) \backslash \backslash T S$. freq $\backslash \backslash 0,1 \backslash 0,-1.6106240074,-0.3289327$ $782,-1.1549254564 \backslash \mathrm{C},-1.6545477485,-0.2075818291,0.1953648773 \backslash \mathrm{C},-3.0011$ $487074,-0.3309467759,0.7123077069 \backslash \mathrm{C},-0.3201167367,-0.1913960147,-1.750$ $6723731 \backslash 0,-0.6518607681,0.0492467242,0.8492317544 \backslash \mathrm{C},-3.2039811525,-0.3$ $450559958,2.0904705749 \backslash \mathrm{H},-3.7736373862,-0.6705180142,0.0304798519 \backslash \mathrm{H}, 0$. $0972020512,0.7991832071,-1.5521079971 \backslash \mathrm{H},-0.4716170993,-0.326109922,-2$. 
$8213087667 \backslash \mathrm{H}, 0.363484603,-0.9535921541,-1.3686671337 \backslash \mathrm{H},-2.4147255962,0$ $.054035574,2.7191107614 \backslash \mathrm{H},-4.2147345118,-0.2319059765,2.4664419893 \backslash \mathrm{Br}$, $-3.0433814225,-2.5240090929,3.0568368072 \backslash \mathrm{C},-1.872734218,3.2046021195,1$ $.8397237417 \backslash \mathrm{C},-2.2519661713,2.6750414649,0.6287105611 \backslash \mathrm{C},-3.4867833806$, $2.064123767,0.2893225788 \backslash \mathrm{C},-4.7231977838,2.2442328089,0.9605998051 \backslash \mathrm{C},-$ $4.9387976388,2.6995560766,2.2396846867 \backslash \mathrm{C},-3.9793218489,3.0906469562,3$. $2061868489 \backslash \mathrm{C},-2.6299072377,3.3047840037,3.0329596273 \backslash \mathrm{H},-1.4973634712,2$ $.672741095,-0.1537856567 \backslash \mathrm{H},-3.5641701733,1.7149138698,-0.7357348124 \backslash \mathrm{H}$, $-5.603768047,1.9380361297,0.4011309454 \backslash \mathrm{H},-5.973968926,2.7269492336,2.5$ $700022127 \backslash \mathrm{H},-4.3719117039,3.2910580633,4.1996397804 \backslash \mathrm{H},-2.0832950899,3$. $6511783128,3.9060038292 \backslash \mathrm{H},-0.8508348272,3.569881147,1.9013732555 \backslash \backslash$ Vers ion $=\mathrm{ES} 64 \mathrm{~L}-\mathrm{G} 16 \mathrm{RevA} .03 \backslash \mathrm{State}=1-\mathrm{A} \backslash \mathrm{HF}=-3151.0352486 \backslash \mathrm{RMSD}=6.784 \mathrm{e}-09 \backslash \mathrm{RMSF}=4$. $514 \mathrm{e}-06 \backslash \mathrm{Dipole}=-1.4086461,6.1420562,-1.4868729 \backslash$ Quadrupole $=4.8338376,-8$ $.0879692,3.2541316,-3.7317152,-3.7852046,14.122722 \backslash \mathrm{PG}=\mathrm{C} 01 \quad[\mathrm{X}(\mathrm{C} 11 \mathrm{H} 13 \mathrm{Br} 1$ 02 ) $] \backslash \backslash @$

\section{4a}

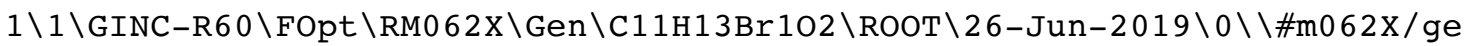
n 6D OPT Freq=Noraman INT (grid=ultrafine) SCRF=(SMD, Solvent=dichloroet hane $) \backslash \backslash$ product.smd $\backslash \backslash 0,1 \backslash 0,-0.786013652,0.3620787496,1.0153735165 \backslash \mathrm{C},-1$. $3964216761,-0.503234838,0.20378529 \backslash C,-2.9005858585,-0.2963359267,0.172$ $6271411 \backslash 0,-0.8114872235,-1.3654234729,-0.4164315552 \backslash \mathrm{C},-3.4263414094,-0$ $.484658602,-1.2399700394 \backslash \mathrm{H},-3.1155887199,0.7179804925,0.5208512042 \backslash \mathrm{H},-$ $3.1094752698,-1.4322017242,-1.6737373763 \backslash \mathrm{H},-4.5116718804,-0.3973873056$ $,-1.2688880635 \backslash \mathrm{Br},-2.7376955194,0.9053173711,-2.4431509393 \backslash \mathrm{C},-3.616909$ $8986,-1.2797621825,1.1289596469 \backslash \mathrm{C},-3.2289930346,-1.088780294,2.5729958$ $08 \backslash C,-3.9721490577,-1.6207798607,3.5648170133 \backslash C,-5.0783023215,-2.53866$ $79052,3.3705298579 \backslash C,-5.172372096,-3.464532383,2.3755618325 \backslash C,-4.18016$ $87377,-3.6913619587,1.3423692729 \backslash \mathrm{C},-3.3930573281,-2.7359082703,0.80669$ $79355 \backslash \mathrm{H},-4.6953259568,-1.0758948841,1.0427579642 \backslash \mathrm{H},-2.3513032914,-0.48$ $984508,2.8095312052 \backslash \mathrm{H},-3.7247684639,-1.3689113709,4.5942948748 \backslash \mathrm{H},-5.82$ $29979485,-2.578649055,4.1628686462 \backslash \mathrm{H},-5.9857428021,-4.1846090204,2.438$ $1244335 \backslash \mathrm{H},-4.0564380549,-4.7187679232,1.0051573248 \backslash \mathrm{H},-2.5909744452,-3$. $0089564554,0.1238987365 \backslash \mathrm{C}, 0.635750043,0.2039404217,1.1455338029 \backslash \mathrm{H}, 0.95$ $60340135,0.9866527781,1.8308002035 \backslash \mathrm{H}, 1.1172317265,0.3255516515,0.17333$ $19612 \backslash \mathrm{H}, 0.8682518629,-0.781734952,1.5539303024 \backslash \backslash$ Version=ES64L-G16RevA. $03 \backslash$ State $=1-A \backslash H F=-3151.0982313 \backslash \mathrm{RMSD}=4.031 \mathrm{e}-09 \backslash \mathrm{RMSF}=3.014 \mathrm{e}-06 \backslash \mathrm{Dipole}=-0$. $347073,-0.1207965,1.3204931 \backslash$ Quadrupole $=5.1724787,-2.1983563,-2.9741224$ $, 6.0499058,2.5399079,2.5884049 \backslash \mathrm{PG}=\mathrm{C} 01[\mathrm{X}(\mathrm{C} 11 \mathrm{H} 13 \mathrm{Br} 1 \mathrm{O} 2)] \backslash \backslash @$

\section{References}

1. Gaussian 16, Revision A.03, Frisch, M. J.; Trucks, G. W.; Schlegel, H. B.; Scuseria, G. E.; Robb, M. A.; Cheeseman, J. R.; Scalmani, G.; Barone, V.; Petersson, G. A.; Nakatsuji, H.; Li, X.; Caricato, M.; Marenich, A. V.; Bloino, J.; Janesko, B. G.; Gomperts, R.; Mennucci, B.; Hratchian, H. P.; Ortiz, J. V.; Izmaylov, A. F.; Sonnenberg, J. L.; WilliamsYoung, D.; Ding, F.; Lipparini, F.; Egidi, F.; Goings, J.; Peng, B.; Petrone, A.; Henderson, T.; Ranasinghe, D.; Zakrzewski, V. G.; Gao, J.; Rega, N.; Zheng, G.; Liang, W.; Hada, M.; Ehara, M.; Toyota, K.; Fukuda, R.; Hasegawa, J.; Ishida, M.; Nakajima, T.; Honda, Y.; Kitao, O.; Nakai, H.; Vreven, T.; Throssell, K.; Montgomery, J. A., Jr.; Peralta, J. E.; Ogliaro, F.; Bearpark, M. J.; Heyd, J. J.; Brothers, E. N.; Kudin, K. N.; Staroverov, V. N.; Keith, T. A.; Kobayashi, R.; Normand, J.; Raghavachari, K.; Rendell, A. P.; Burant, J. C.; Iyengar, S. S.; Tomasi, J.; Cossi, M.; Millam, J. M.; Klene, M.; Adamo, C.; Cammi, R.; Ochterski, J. W.; Martin, R. L.; Morokuma, K.; Farkas, O.; Foresman, J. B.; Fox, D. J. Gaussian, Inc., Wallingford CT, 2016.

2. Zhao, Y.; Truhlar, D. G., Theor. Chem. Acc. 2008, 120, 215-241. 
3. Marenich, A. V.; Cramer, C. J.; Truhlar, D. G., J. Phys. Chem. B 2009, 113, 63786396.

4. $\quad$ Ho, J.; Ertem, M. Z., J. Phys. Chem. B 2016, 120, 1319-1329.

5. Fukui, K., Acc. Chem. Res. 1981, 14, 363-368.

6. Wiberg, K. B. Tetrahedron, 1966, 24, 1083-1096. 


\section{X-ray Crystallography:}

Suitable single crystal of adduct 13 was selected under the polarizing microscope (Leica M165Z), mounted on a MicroMount (MiTeGen, USA) consisting of a thin polymer tip with a wicking aperture. The X-ray diffraction measurements were carried out on a Bruker D8 Quest Single Crystal diffractometer with Photon II detector at $100 \mathrm{~K}$ by using I $\mu \mathrm{S} 3.0$ Microfocus Source with Mo-K $\alpha$ radiation $(\lambda=0.710723 \AA)$. The single crystal, mounted on the goniometer using cryo loops for intensity measurements, was coated with paraffin oil and then quickly transferred to the cold stream using an Oxford Cryo stream 800 attachment. Symmetry related absorption corrections using the program SADABS ${ }^{[1]}$ were applied and the data were corrected for Lorentz and polarisation effects using Bruker APEX3 software. ${ }^{[1]}$ The structure was solved by ShelxT (intrinsic phasing) ${ }^{[2]}$ and the full-matrix least-square refinement was carried out using Shelxl ${ }^{[3]}$ in Olex $2^{[4]}$. The non-hydrogen atoms were refined anisotropically. The molecular graphic was generated using program Olex2. ${ }^{[4]}$

Atomic coordinates, bond lengths and angles and displacement parameters for compound $\mathbf{1 3}$ have been deposited at the Cambridge Crystallographic Data Centre (CCDC No. 1922409). This data can be obtained free-of-charge via www.ccdc.cam.ac.uk/data_request/cif, by emailing data_request@ccdc.cam.ac.uk or by contacting The Cambridge Crystallographic Data Centre, 12 Union Road, Cambridge CB2 1EZ, UK; fax +44 1223336033.

[1] Bruker (2016). APEX3, SAINT and SADABS. Bruker AXS Inc., Madison, Wisconsin, USA.

[2] Sheldrick, G. M. (2015a). Acta Cryst. A71, 3-8.

[3] Sheldrick, G. M. (2015b). Acta Cryst. C71, 3-8.

[4] Dolomanov, O.V.; Bourhis, L.J.; Gildea, R.J.; Howard, J.A.K.; Puschmann, H., OLEX2: A complete structure solution, refinement and analysis program (2009). J. Appl. Cryst., 42, 339-341. 
Table S2. Crystallographic Experimental details

\section{Structure of $\mathbf{1 3}$}

Crystal data

Chemical formula

$\mathrm{C}_{15} \mathrm{H}_{16} \mathrm{BrNO}_{4}$

$M_{\mathrm{r}}$

354.20

Crystal system, space group

Monoclinic, $P 2_{1} / c$

Temperature (K) $\quad 150$

$a, b, c(\AA) \quad 9.0366(6), 13.2505(8), 12.5159(9)$

$\beta\left(^{\circ}\right) \quad 103.546(3)$

$V\left(\AA^{3}\right) \quad 1456.96(17)$

Z 4

Radiation type Mo $K \alpha$

$\mu\left(\mathrm{mm}^{-1}\right) \quad 2.84$

Crystal size $(\mathrm{mm}) \quad 0.22 \times 0.07 \times 0.04$

Data collection

Diffractometer

Bruker D8Quest

Multi-scan

Absorption

SADABS2016/2 (Bruker,2016/2) was used for absorption correction.

correction wR2(int) was 0.1547 before and 0.1024 after correction. The Ratio of minimum to maximum transmission is 0.5697 . The $\lambda / 2$ correction factor is Not present.

$T_{\text {min },} T_{\text {max }} \quad 0.425,0.746$

No. of measured, independent and observed $[I>2 \sigma(I)]$

45974, 3358, 2923

reflections

\begin{tabular}{l|l}
$R_{\text {int }}$ & 0.093
\end{tabular}

$(\sin \theta / \lambda)_{\max }\left(\AA^{-1}\right) \quad 0.651$

Refinement

$R\left[F^{2}>\right.$

$\left.2 \sigma\left(F^{2}\right)\right], w R\left(F^{2}\right), S$

$0.044,0.125,1.09$

No. of reflections 3358

No. of parameters 191

$\mathrm{H}$-atom treatment $\mathrm{H}$-atom parameters constrained

$\Delta \mathrm{Q}_{\max }, \Delta \varrho_{\text {min }}\left(\mathrm{e} \AA^{-3}\right) \quad 0.87,-0.80$

Computer programs: SAINT V8.38A (?, 2016), SHELXT 2014/5 (Sheldrick, 2014), SHELXL (Sheldrick, 2015), Olex2 (Dolomanov et al., 2009). 


\section{NMR Spectra}

Methyl 3-bromo-2-(cyclohepta-2,4,6-trien-1-yl)propanoate (4a); ${ }^{\mathbf{H}} \mathbf{H} \mathbf{N M R}(400 \mathrm{MHz}$, $\left.\mathrm{CDCl}_{3}\right),{ }^{13} \mathbf{C} \mathbf{N M R}\left(100 \mathrm{MHz}, \mathrm{CDCl}_{3}\right)$.<smiles>CC(=O)C(CBr)C1C=CC=CC=C1</smiles>

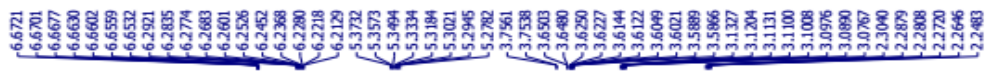
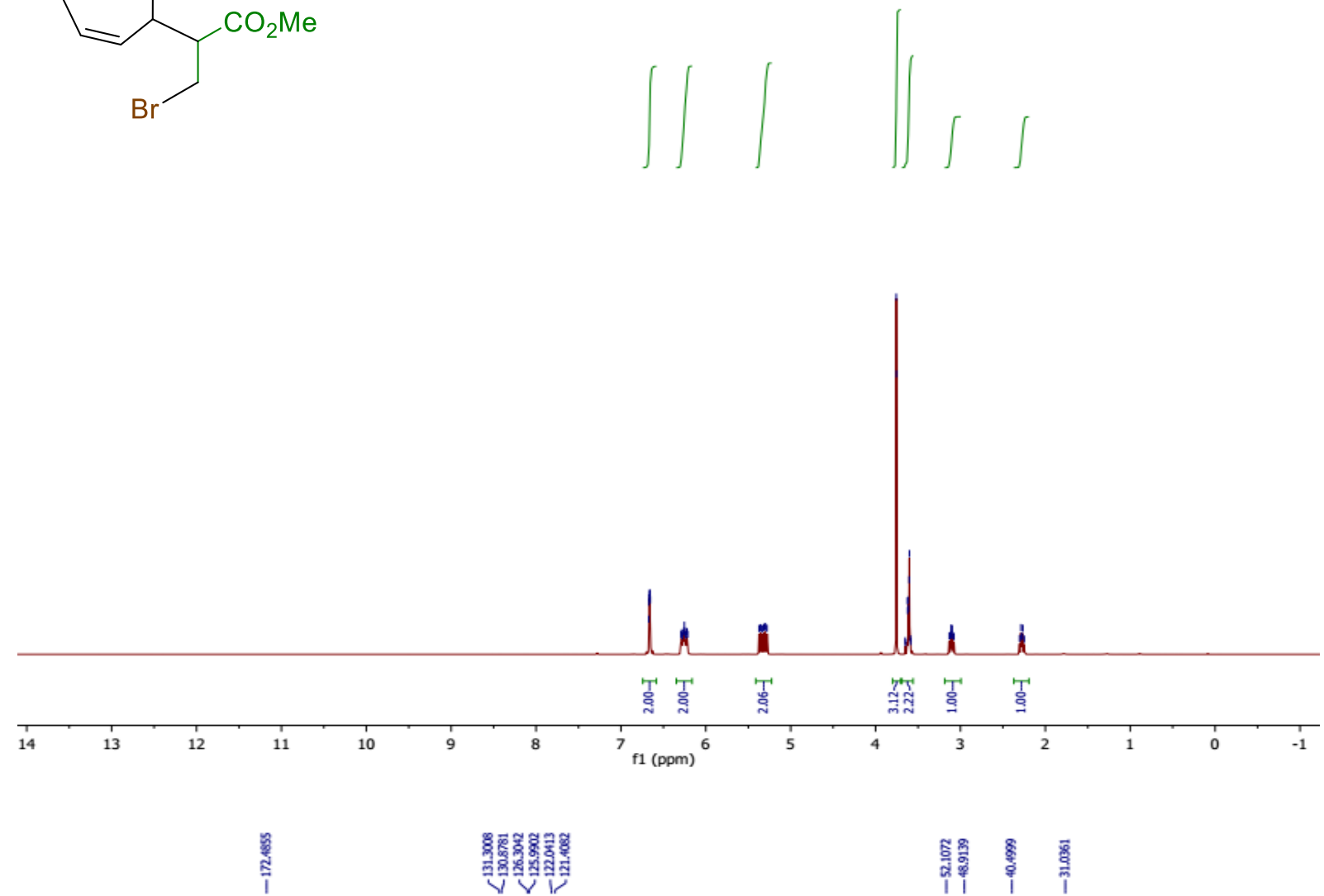

紧 1

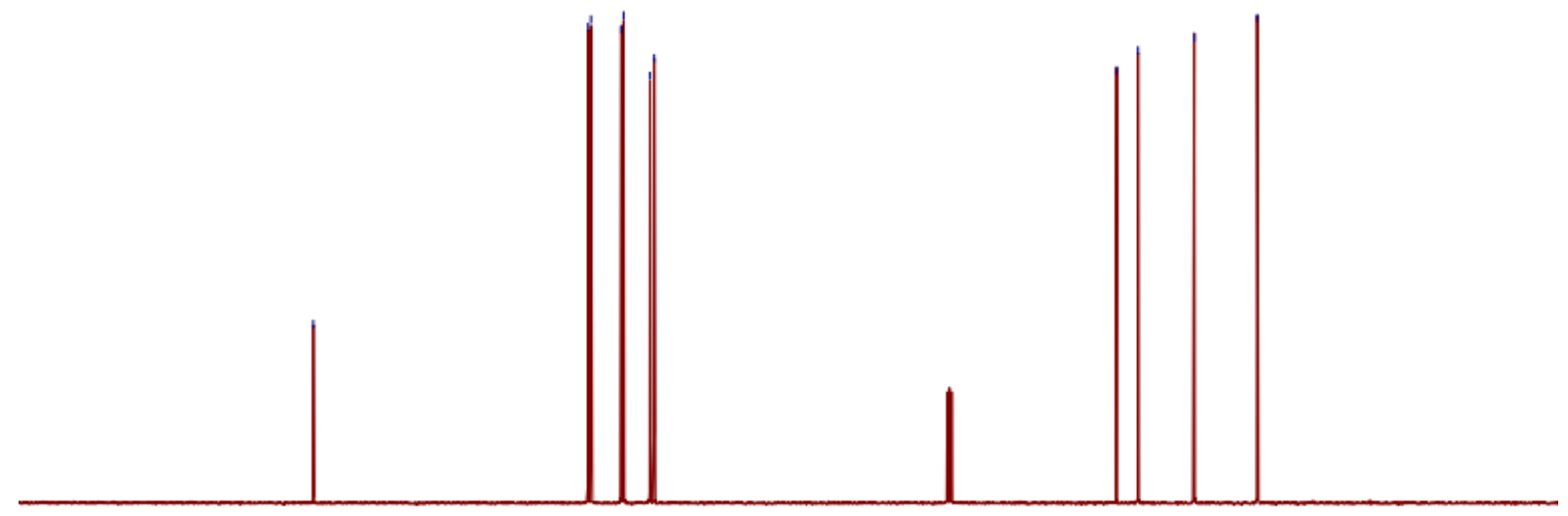

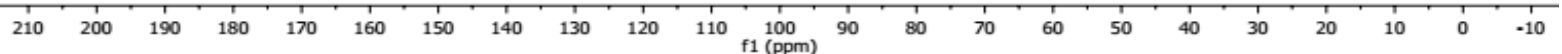


Methyl 3-chloro-2-(cyclohepta-2,4,6-trien-1-yl)propanoate (4aCl); ${ }^{1} \mathbf{H}$ NMR (400 $\mathbf{M H z}$, $\left.\mathrm{CDCl}_{3}\right),{ }^{13} \mathrm{C}$ NMR (100 MHz, $\left.\mathrm{CDCl}_{3}\right)$.
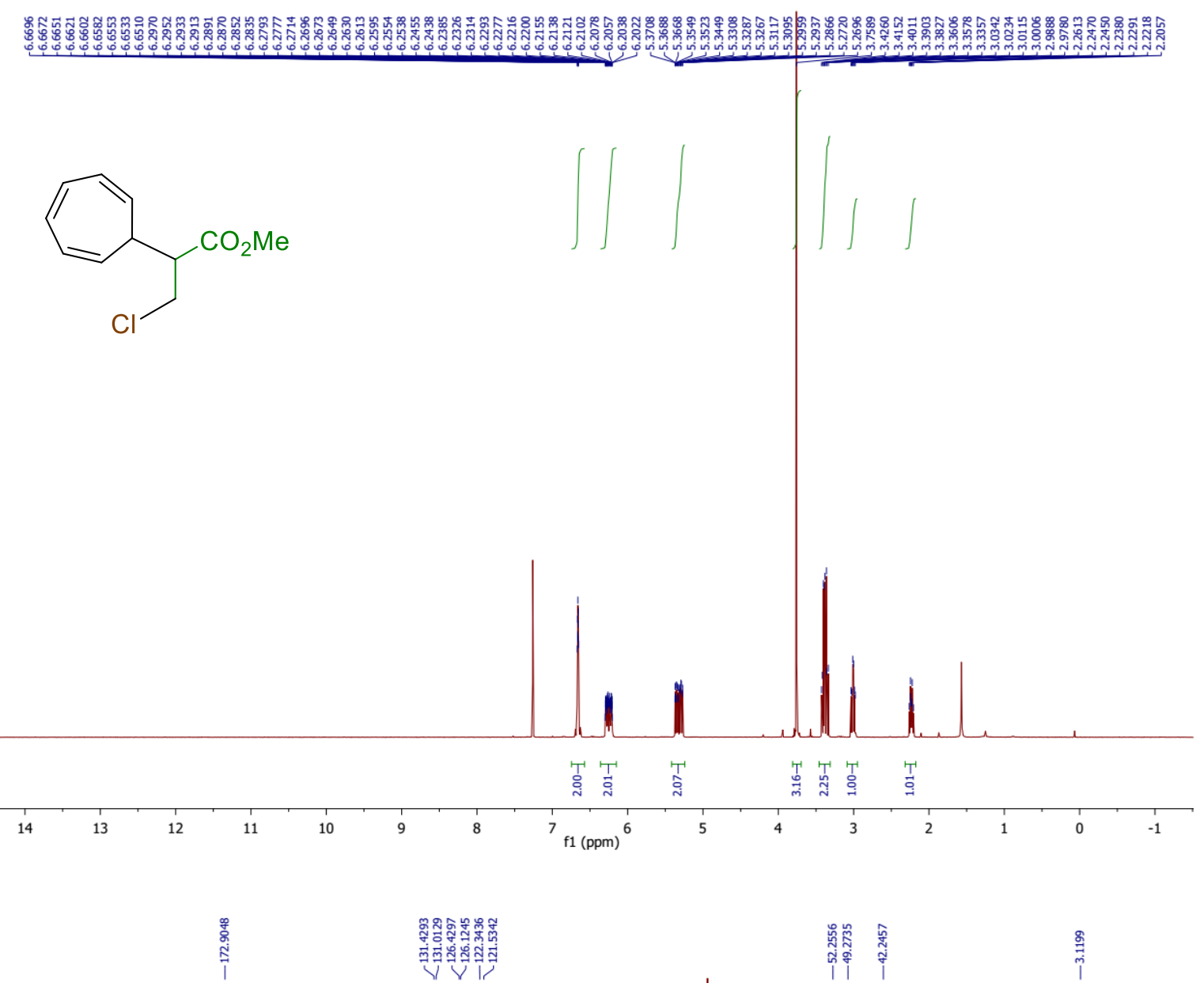

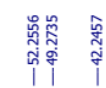

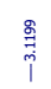

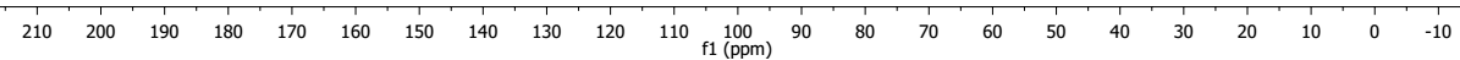


Methyl 2-(cyclohepta-2,4,6-trien-1-yl)-3-iodopropanoate (4aI); ${ }^{1} \mathbf{H}$ NMR (400 MHz, $\mathrm{CDCl}_{3}$ ), ${ }^{13}$ C NMR (100 MHz, $\left.\mathrm{CDCl}_{3}\right)$.

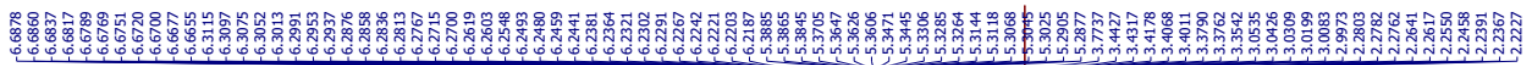<smiles>CC(=O)C(CI)C1C=CC=CC=C1</smiles>
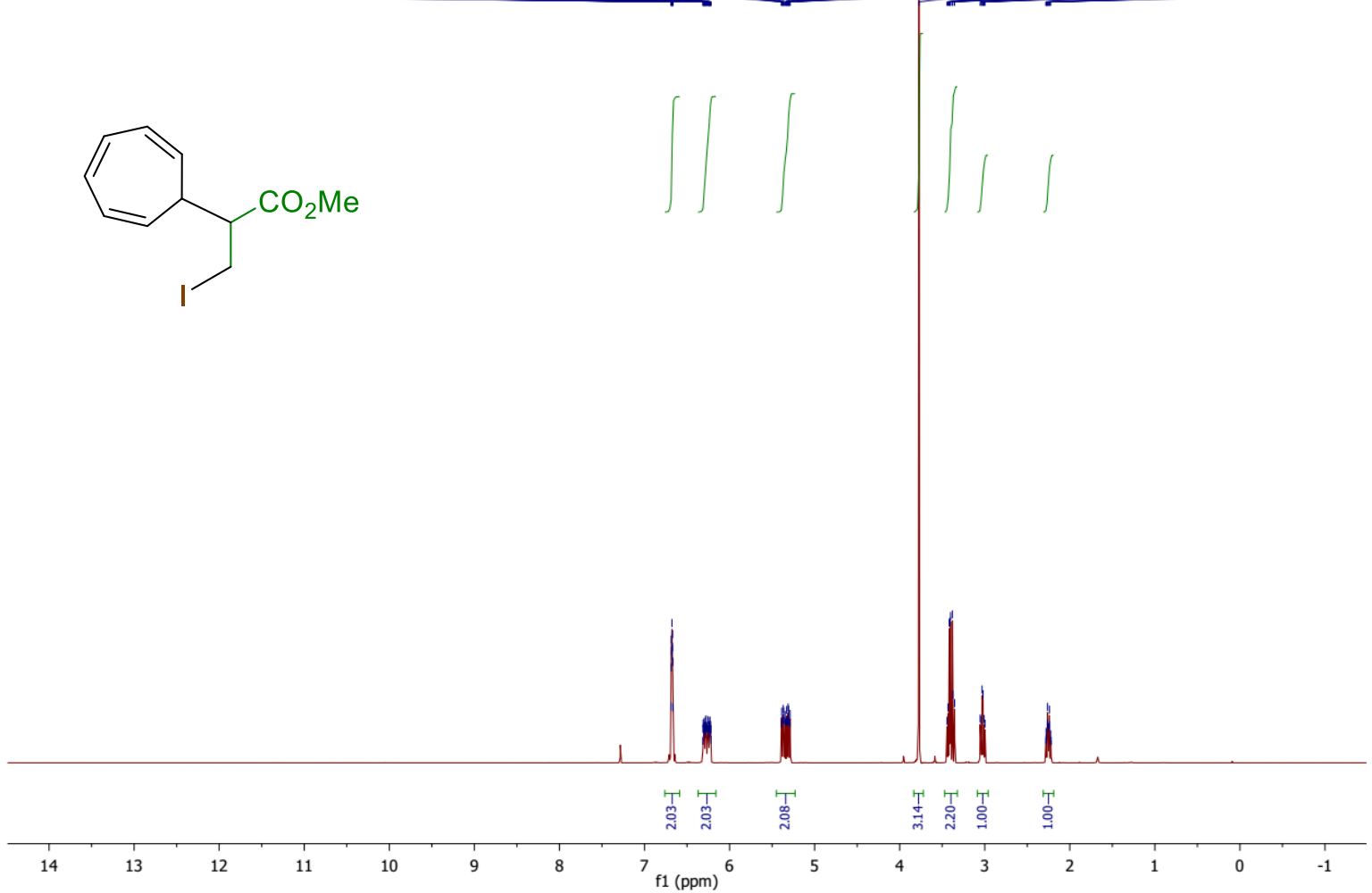

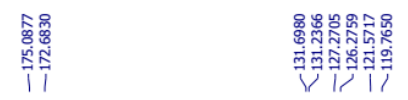

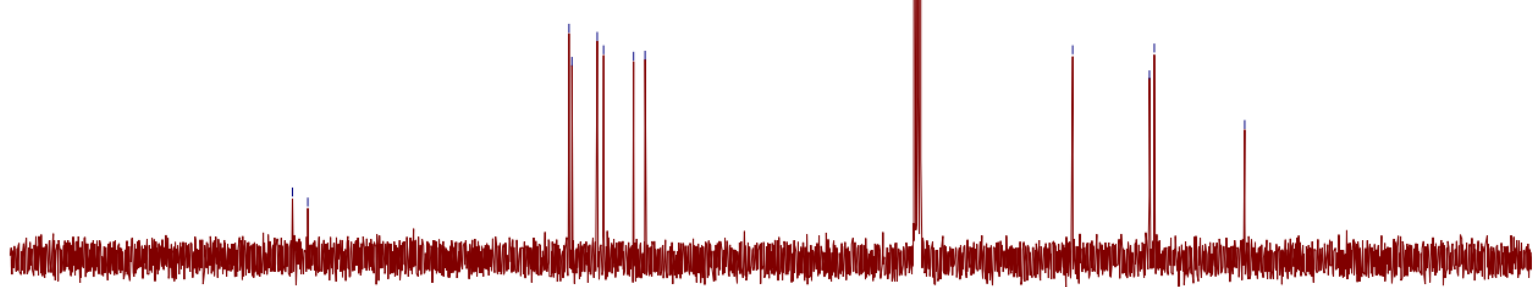

$\begin{array}{lllllllllllllllllllllllll}210 & 200 & 190 & 180 & 170 & 160 & 150 & 140 & 130 & 120 & 110 & \begin{array}{c}100 \\ \mathrm{f} 1(\mathrm{ppm})\end{array} & 90 & 80 & 70 & 60 & 50 & 40 & 30 & 20 & 10 & 0 & -10\end{array}$ 
Ethyl 3-bromo-2-(cyclohepta-2,4,6-trien-1-yl)propanoate (4b); ${ }^{1} \mathbf{H} \mathbf{N M R}\left(400 \mathrm{MHz}, \mathrm{CDCl}_{3}\right)$, ${ }^{13} \mathbf{C} \mathbf{N M R}\left(100 \mathrm{MHz}, \mathrm{CDCl}_{3}\right)$.
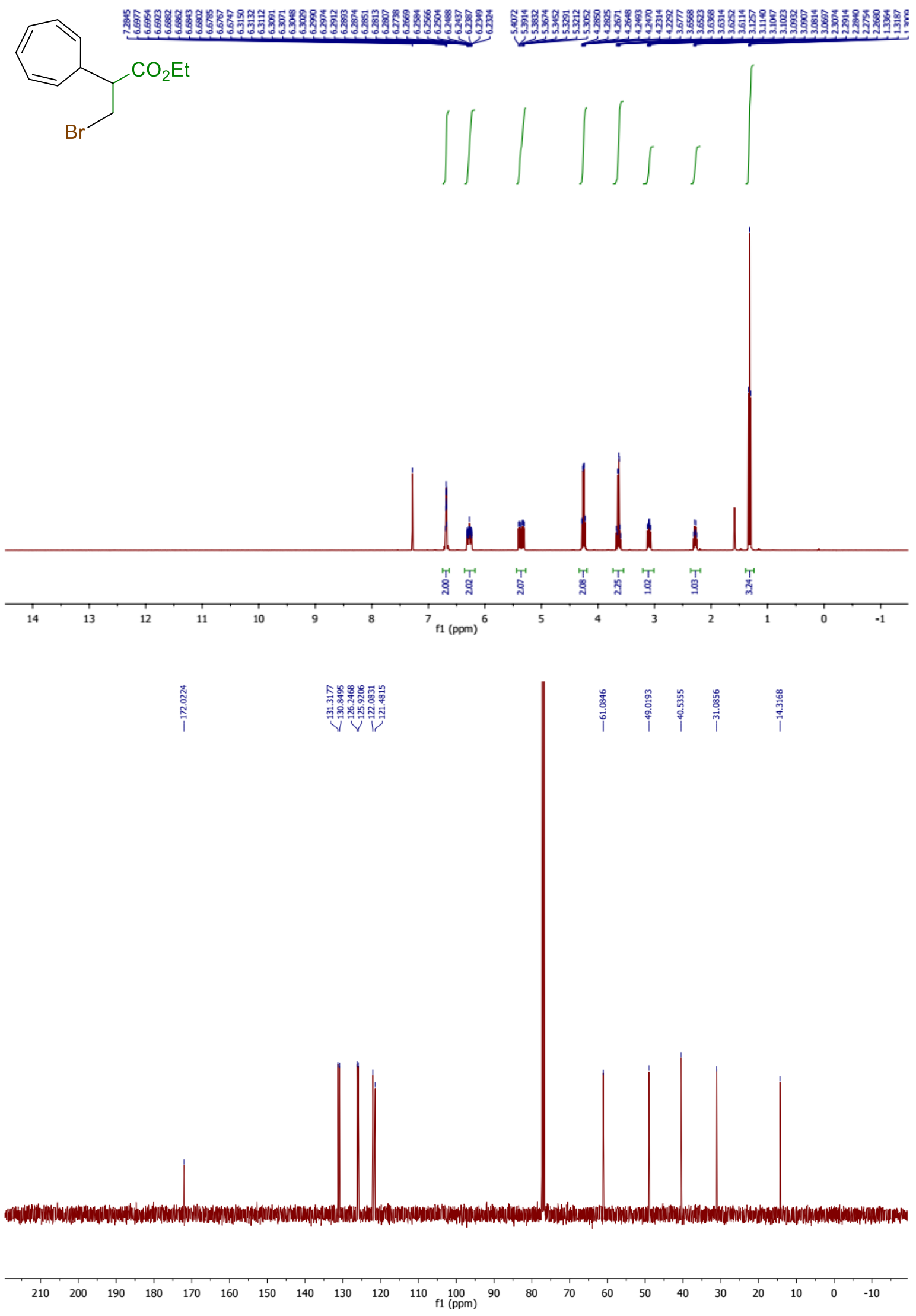

S35 
Butyl 3-bromo-2-(cyclohepta-2,4,6-trien-1-yl)propanoate (4c); ${ }^{1} \mathbf{H}$ NMR (400 MHz, CDCl 3 ), ${ }^{13}$ C NMR (100 MHz, $\left.\mathrm{CDCl}_{3}\right)$.

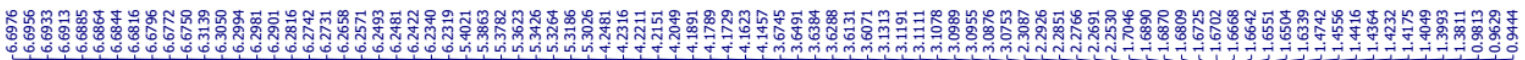<smiles>CCCCC(CBr)C(CBr)C1C=CC=CC=C1</smiles>
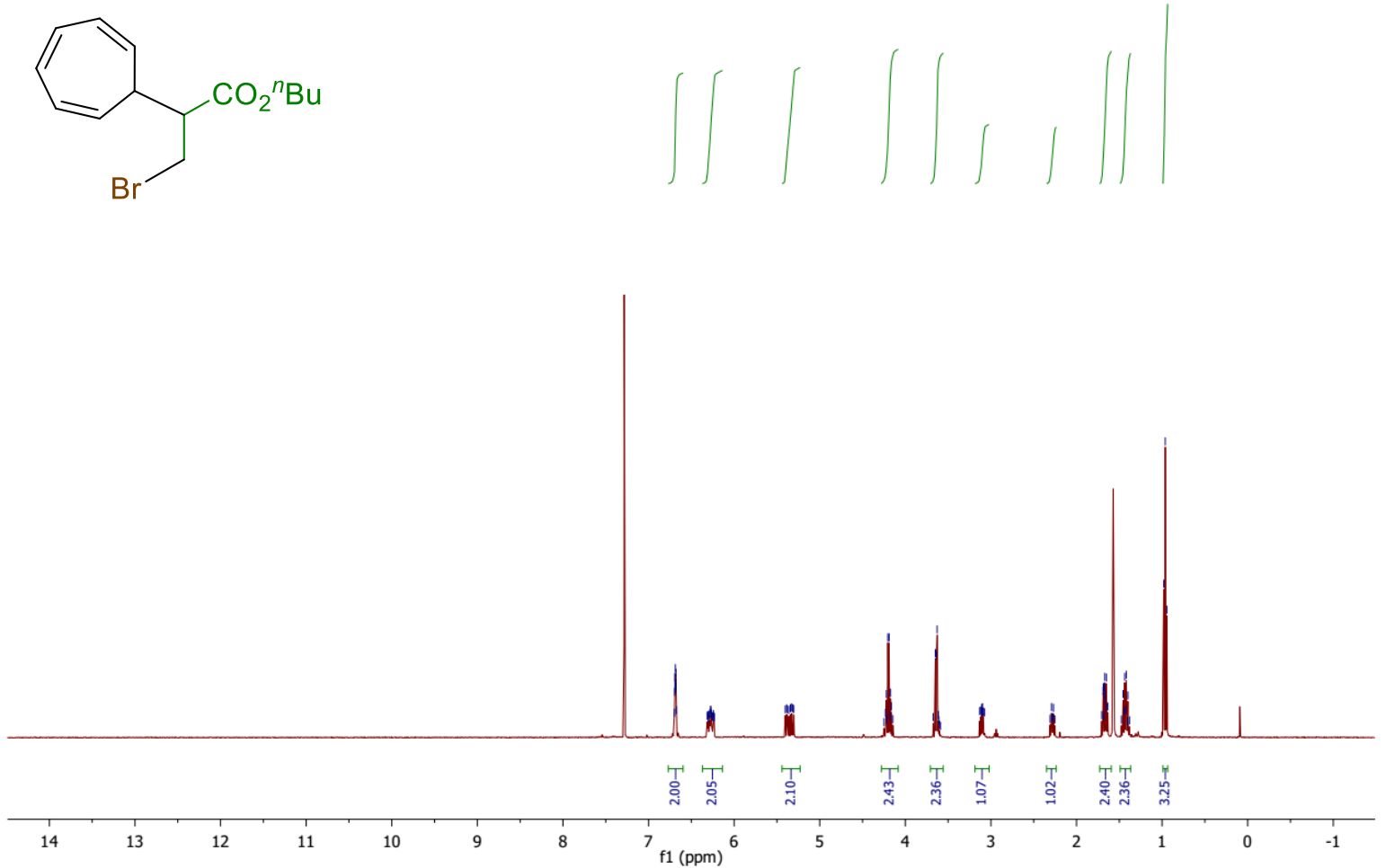
Isobutyl 3-bromo-2-(cyclohepta-2,4,6-trien-1-yl)propanoate (4d); ${ }^{1} \mathbf{H}$ NMR (400 MHz, $\left.\mathrm{CDCl}_{3}\right),{ }^{13} \mathbf{C} \mathbf{N M R}\left(100 \mathrm{MHz}, \mathrm{CDCl}_{3}\right)$.

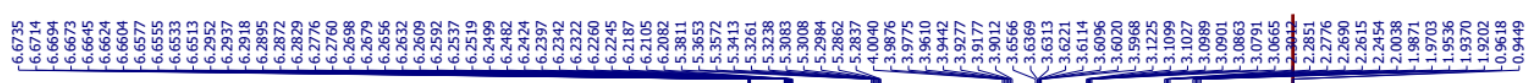<smiles>CC(C)(C)OC(=O)C(CBr)C1C=CC=CC=C1</smiles>
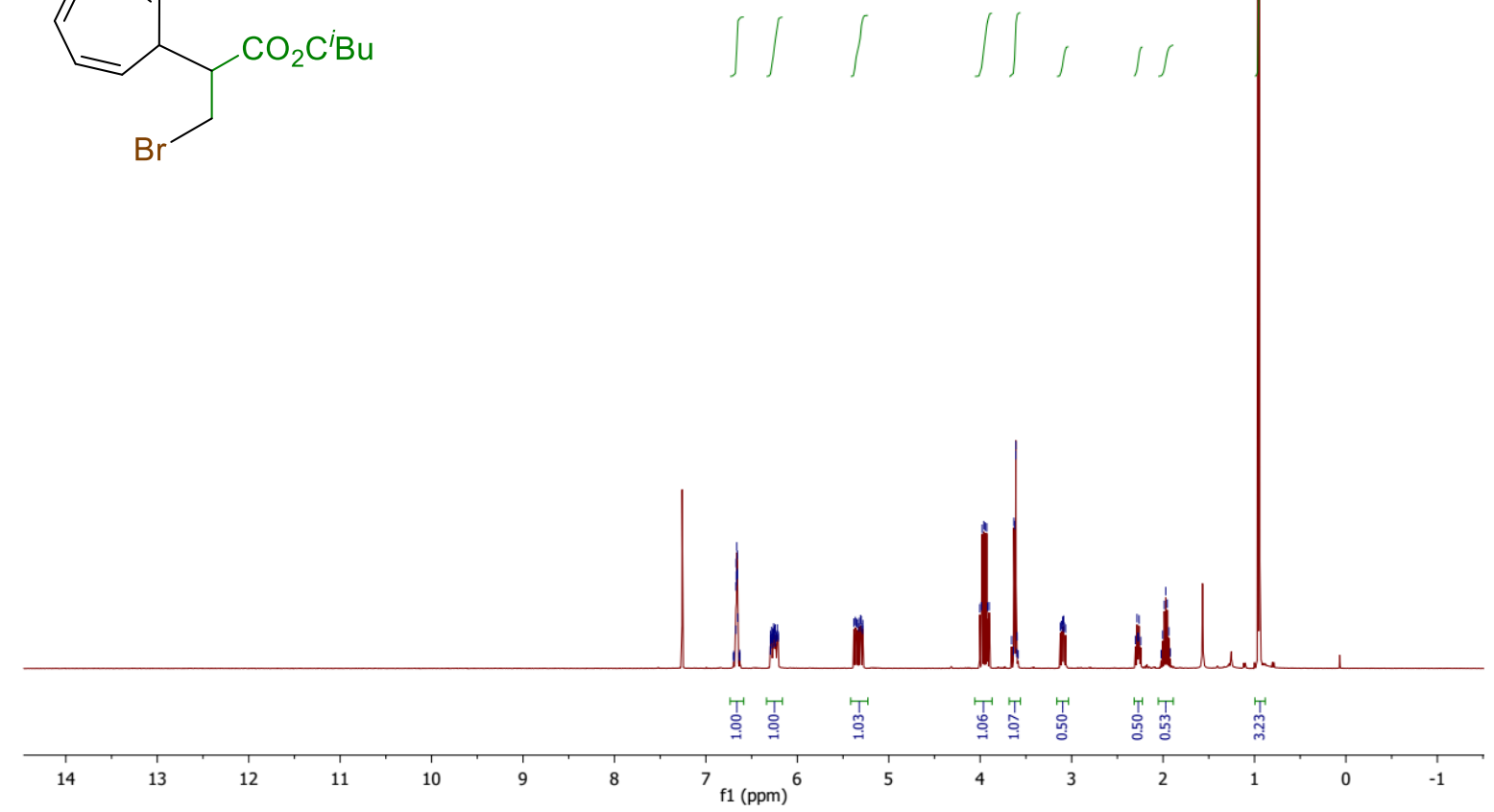

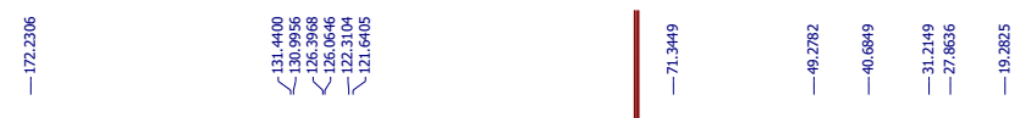

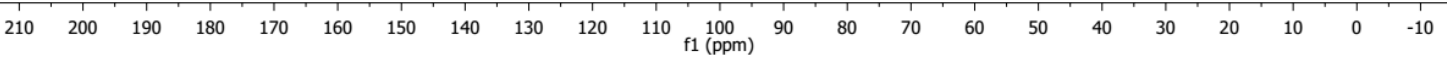


3-Bromo-2-(cyclohepta-2,4,6-trien-1-yl)propanoic acid (4e/4g); ${ }^{\mathbf{H}} \mathbf{H} \mathbf{N M R}\left(400 \mathrm{MHz}, \mathrm{CDCl}_{3}\right)$, ${ }^{13} \mathbf{C}$ NMR (100 MHz, $\left.\mathrm{CDCl}_{3}\right)$.

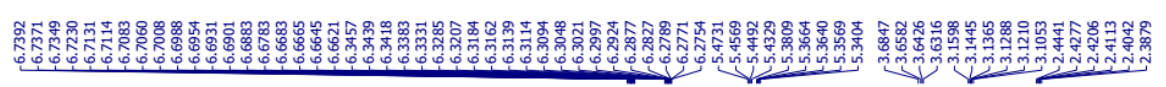<smiles>O=C(O)C(CBr)C1C=CC=CC=C1</smiles>
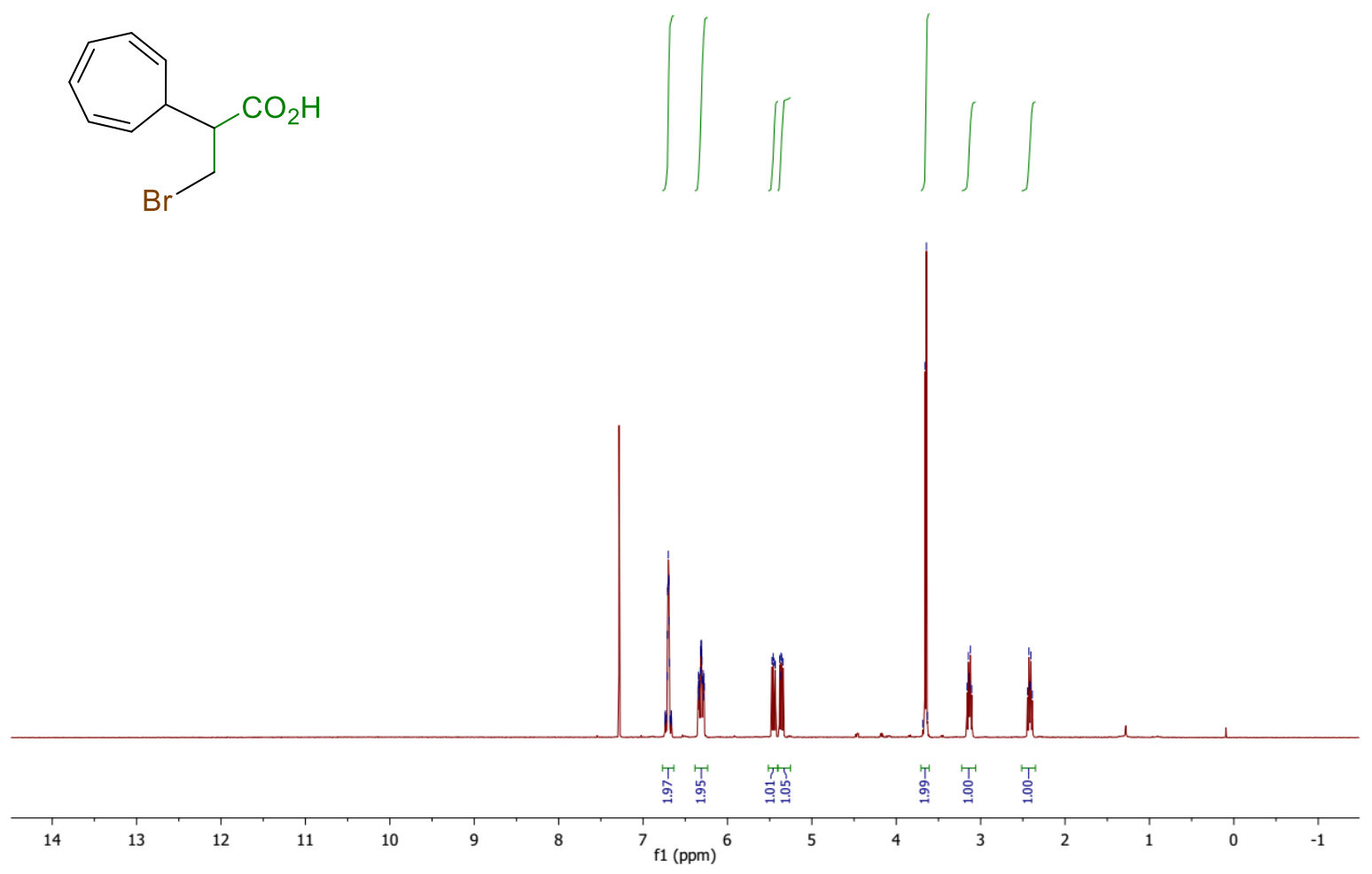

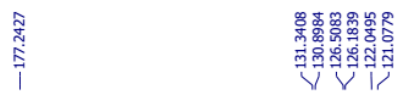

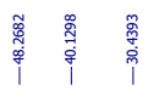


2-Phenoxyethyl 3-bromo-2-(cyclohepta-2,4,6-trien-1-yl)propanoate (4f); ${ }^{\mathbf{H}}$ NMR (400 MHz, $\left.\mathrm{CDCl}_{3}\right),{ }^{13} \mathbf{C} \mathbf{N M R}\left(100 \mathrm{MHz}, \mathrm{CDCl}_{3}\right)$.
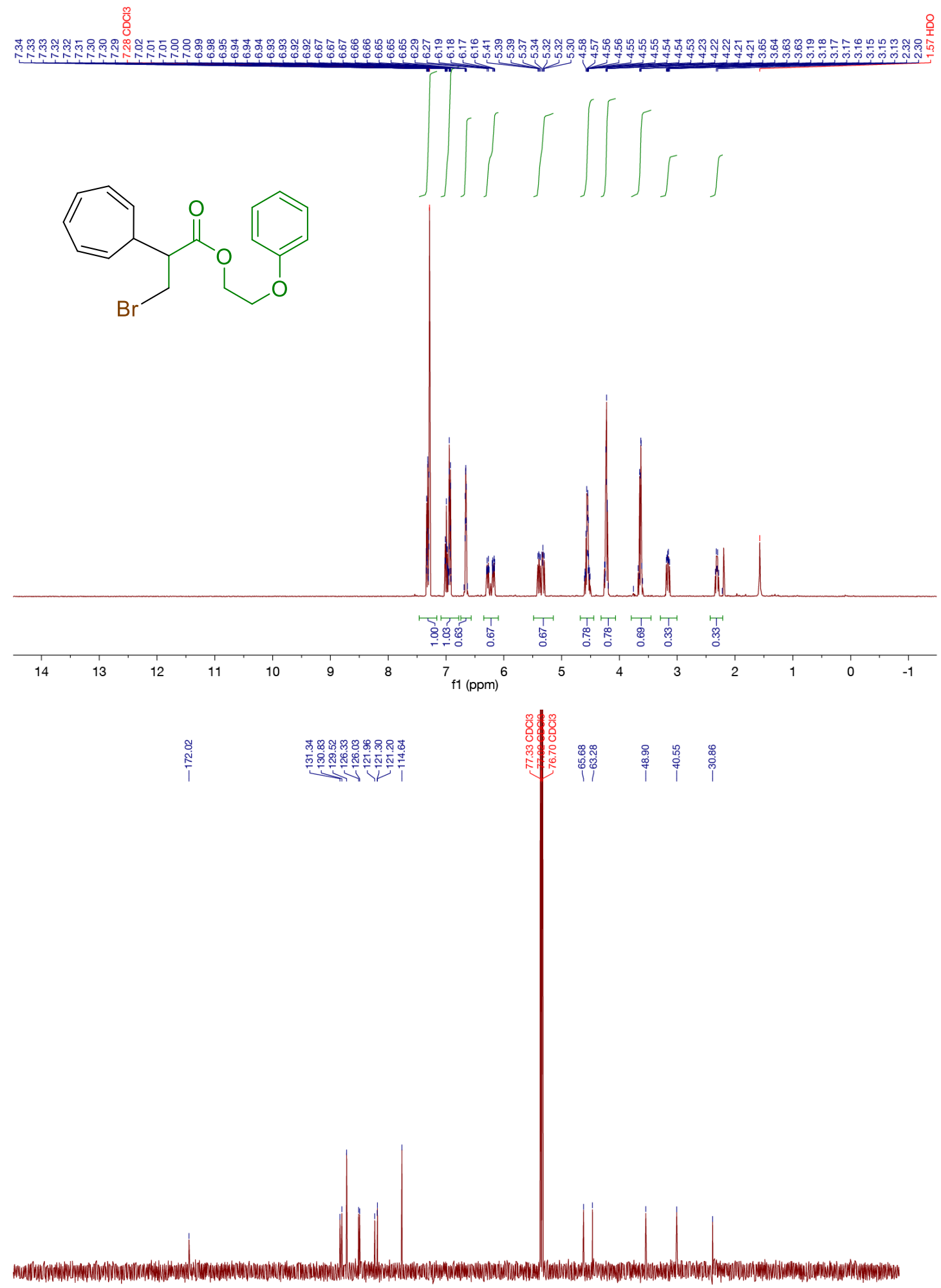

$\begin{array}{llllllllllllllllllllllllll}210 & 200 & 190 & 180 & 170 & 160 & 150 & 140 & 130 & 120 & 110 & 100 & 90 & 80 & 70 & 60 & 50 & 40 & 30 & 20 & 10 & 0 & -10\end{array}$ 
((Oxybis(ethane-2,1-diyl))bis(oxy))bis(ethane-2,1-diyl)bis(3-bromo-2-(cyclohepta-2,4,6trien-1-yl)propanoate) (4h); ${ }^{1} \mathrm{H}$ NMR (400 $\left.\mathrm{MHz}, \mathrm{CDCl}_{3}\right),{ }^{13} \mathrm{C} \mathrm{NMR}\left(100 \mathrm{MHz}, \mathrm{CDCl}_{3}\right)$.

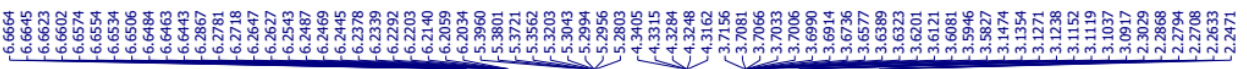<smiles>[Z]C(C)(CC)COCCOC(=O)C(CBr)C1C=CC=CC=C1</smiles>
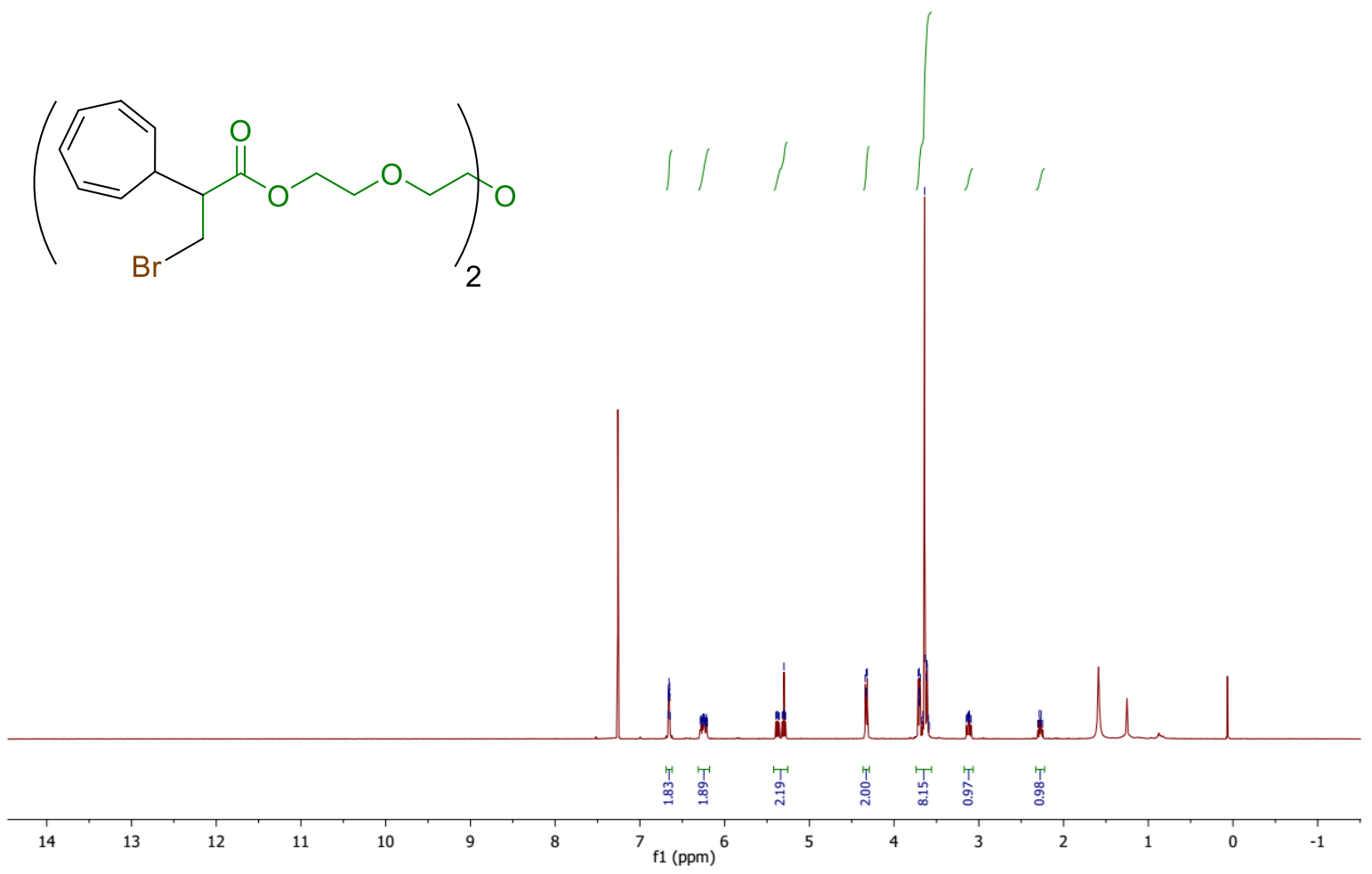

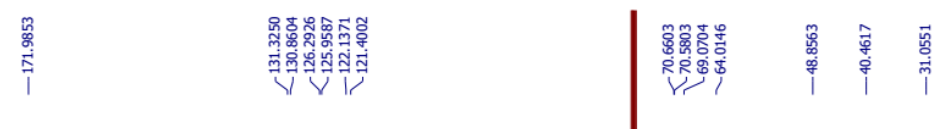

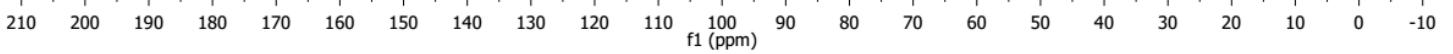


Vinyl 3-bromo-2-(cyclohepta-2,4,6-trien-1-yl)propanoate (4i); ${ }^{1} \mathbf{H}$ NMR (400 $\left.\mathbf{M H z}, \mathbf{C D C l}_{3}\right)$, ${ }^{13} \mathrm{C}$ NMR (100 MHz, $\left.\mathrm{CDCl}_{3}\right)$.

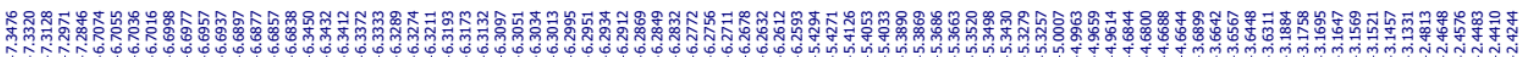<smiles>C=COC(=O)C(CBr)C1C=CC=CC=C1</smiles>
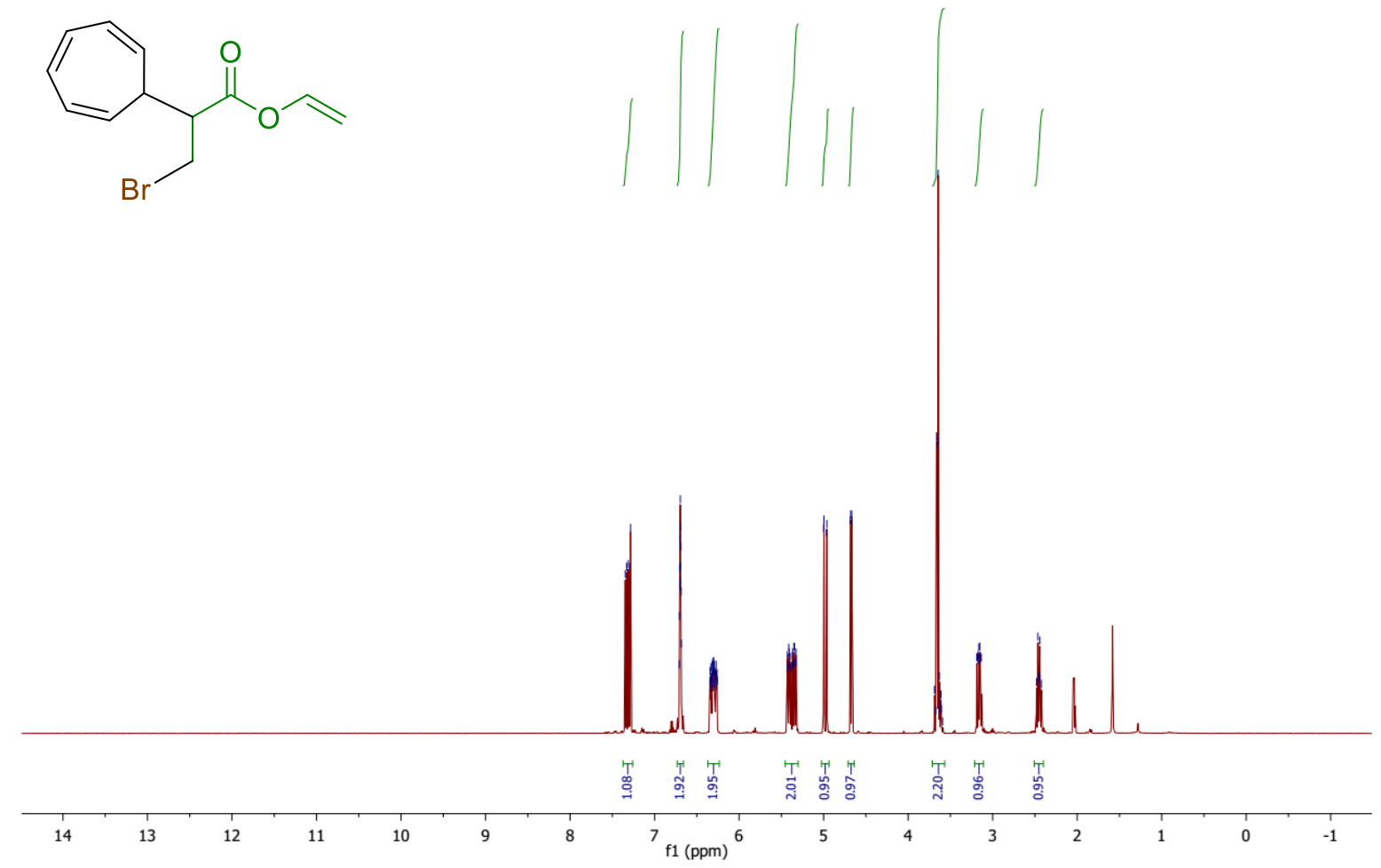

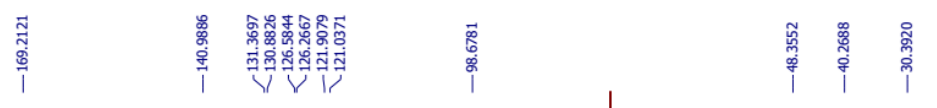

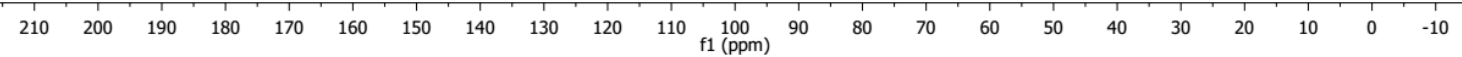


Prop-2-yn-1-yl (Z)-2-(bromomethyl)-3-methylhepta-4,6-dienoate (4j); ${ }^{1} \mathbf{H}$ NMR (400 MHz, $\left.\mathrm{CDCl}_{3}\right),{ }^{13} \mathrm{C}$ NMR (100 MHz, $\left.\mathrm{CDCl}_{3}\right)$.

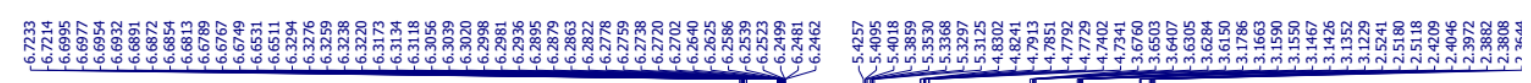<smiles>C#CCOC(=O)C(CBr)C1C=CC=CC=C1</smiles>
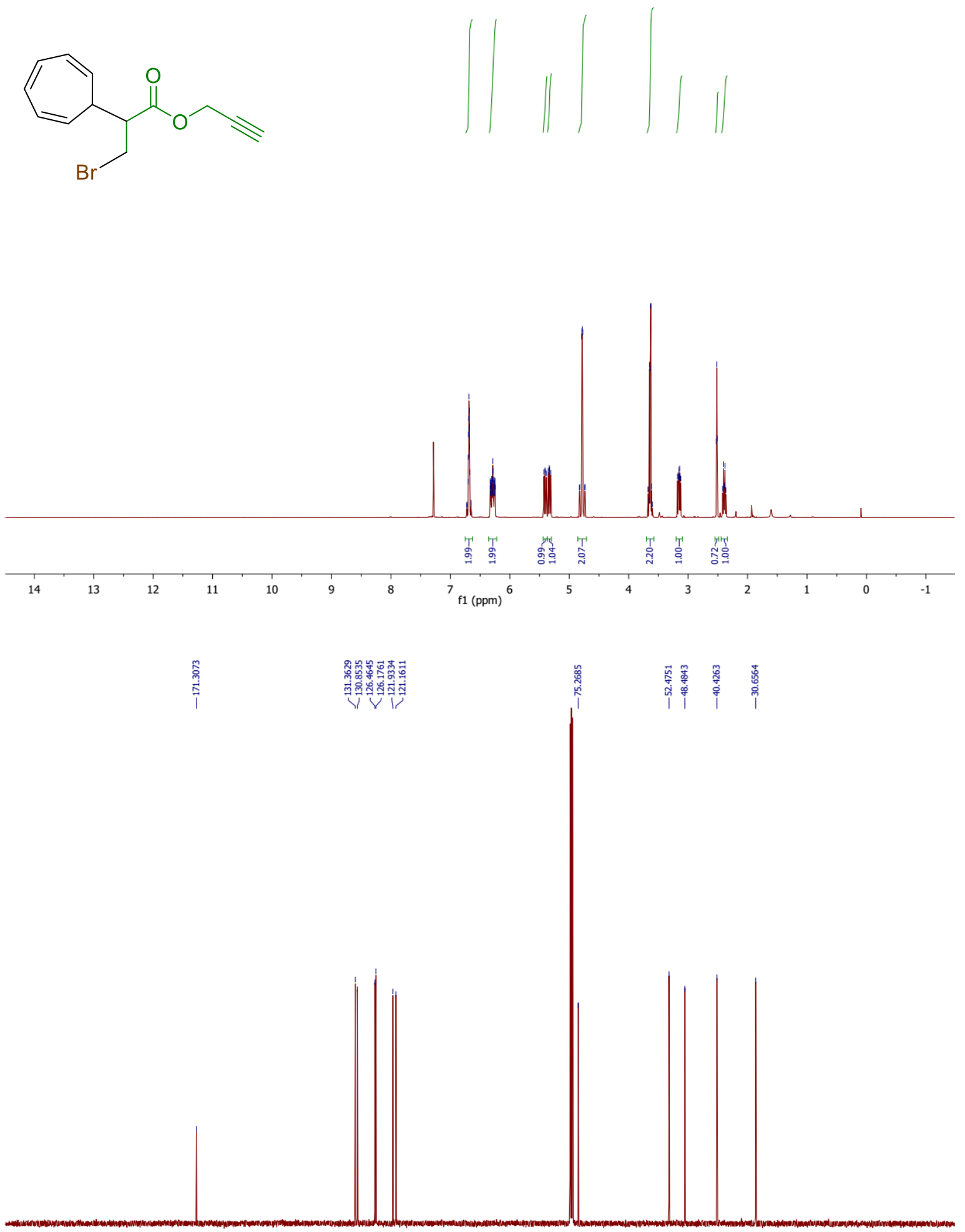

$\begin{array}{lllllllllllllllllllllllllllll}210 & 200 & 190 & 180 & 170 & 160 & 150 & 140 & 130 & 120 & 110 & 100 & 10 & 10 & 70 & 60 & 50 & 40 & 30 & 20 & 10 & 0 & -10\end{array}$ 
3-Bromo-2-(cyclohepta-2,4,6-trien-1-yl)propanamide (4k); ${ }^{1} \mathbf{H}$ NMR (400 MHz, $\left.\mathrm{CDCl}_{3}\right),{ }^{13} \mathrm{C}$ NMR (100 MHz, CDCl $)_{3}$.

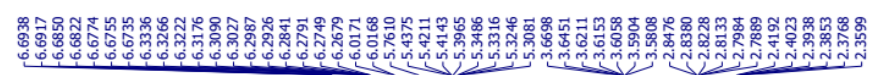<smiles>NC(=O)C(CBr)C1C=CC=CC=C1</smiles>
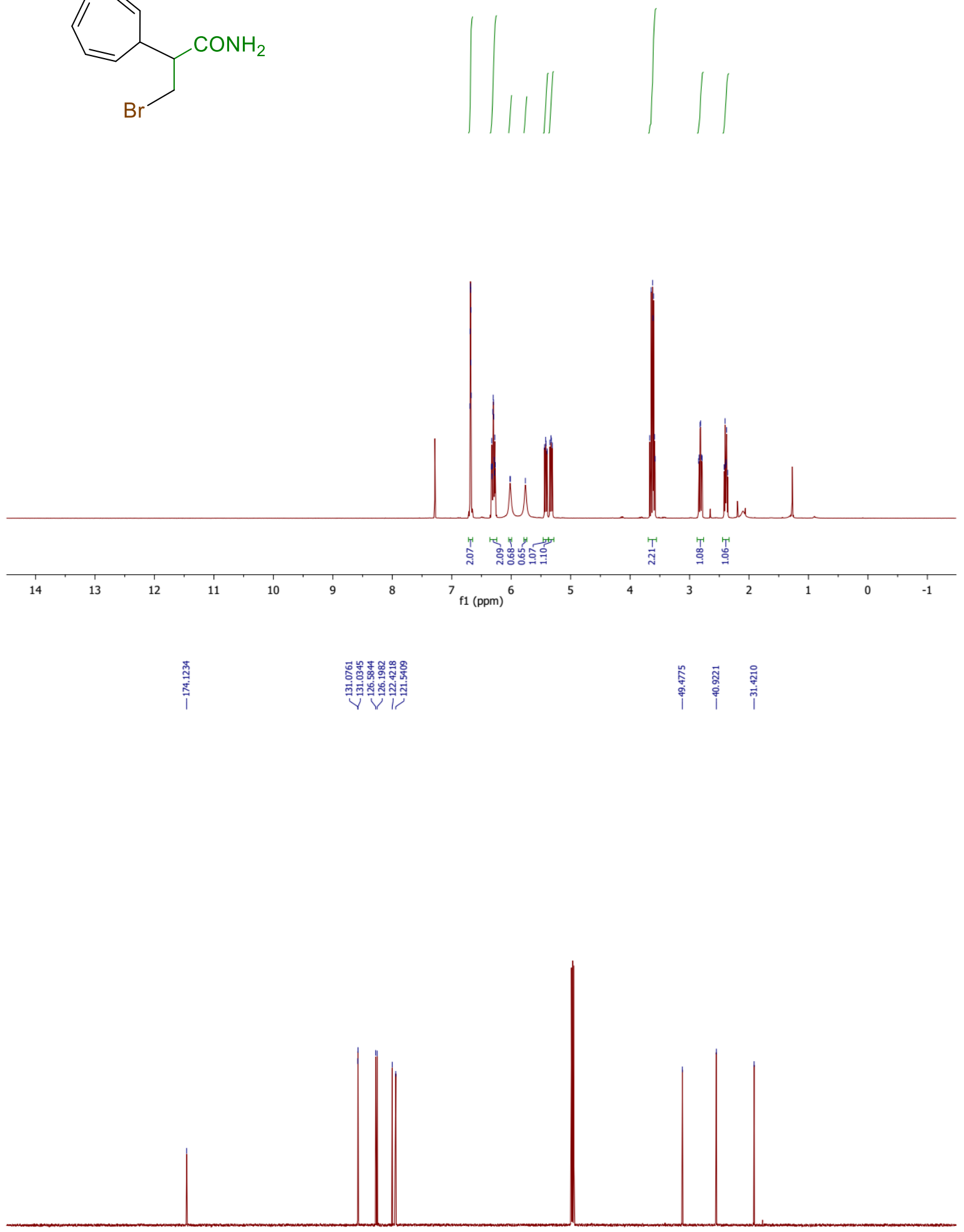

$\begin{array}{llllllllllllllllllllllll}210 & 200 & 190 & 180 & 170 & 160 & 150 & 140 & 130 & 120 & 110 & 100 & 10 & 10 & 10 & 70 & 60 & 50 & 40 & 30 & 20 & 10 & 0 & -10\end{array}$ 
3-Bromo-2-(cyclohepta-2,4,6-trien-1-yl)- $N, N$-dimethylpropanamide (4l); ${ }^{\mathbf{1}} \mathbf{H} \quad$ NMR $\quad$ (400 $\left.\mathrm{MHz}, \mathrm{CDCl}_{3}\right),{ }^{13} \mathrm{C} \mathrm{NMR}\left(100 \mathrm{MHz}, \mathrm{CDCl}_{3}\right)$.

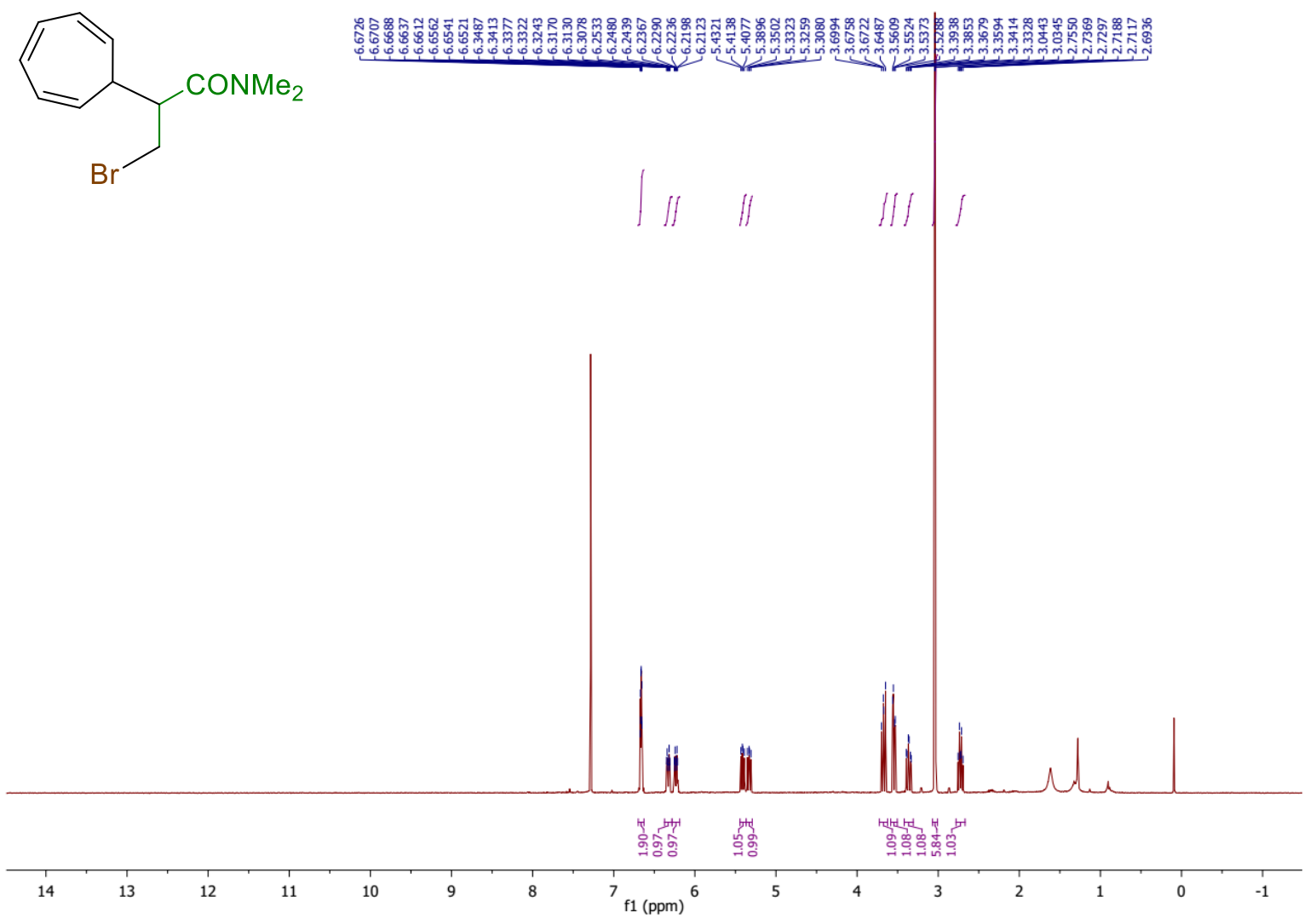

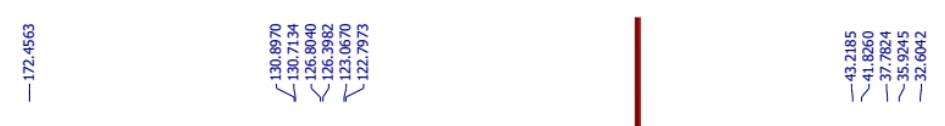

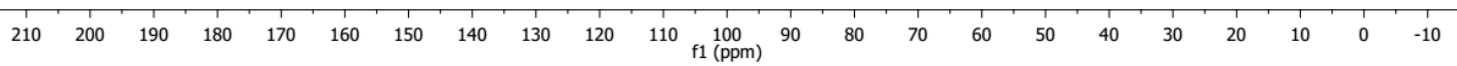


3-Bromo-N,N-dimethylpropanamide (4I-H); ${ }^{1} \mathrm{H}$ NMR (400 MHz, CDCl 3 ), ${ }^{13} \mathrm{C}$ NMR (100 $\left.\mathrm{MHz}, \mathrm{CDCl}_{3}\right)$.<smiles>CC(=O)CCBr</smiles>

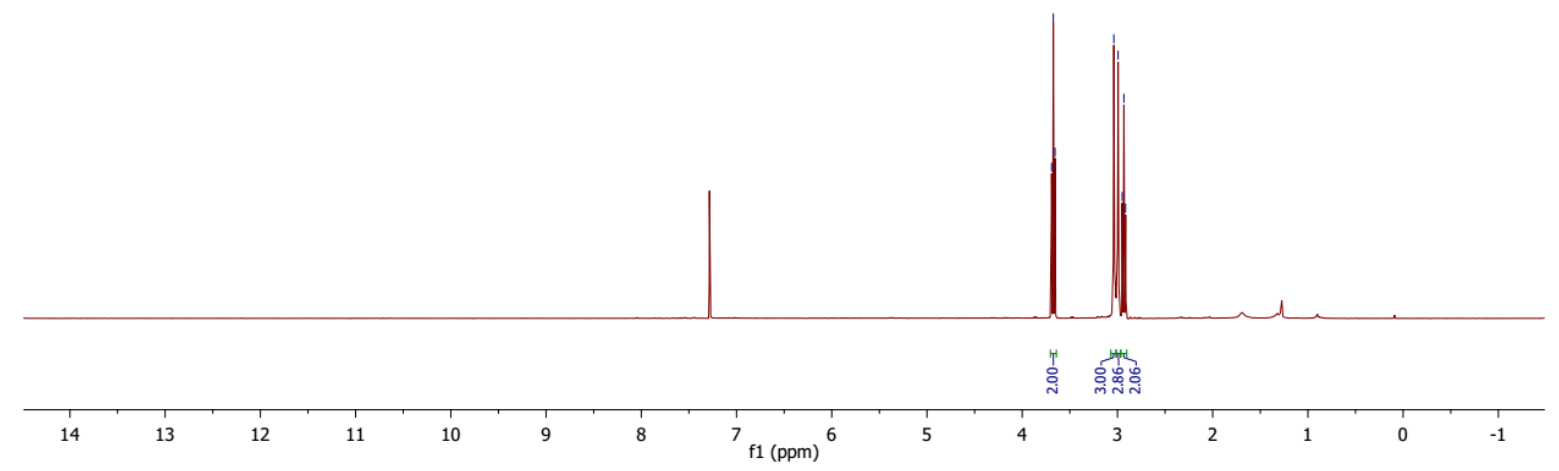


3-Bromo-2-(cyclohepta-2,4,6-trien-1-yl)-1-morpholinopropan-1-one (4m); ${ }^{\mathbf{1}} \mathbf{H}$ NMR (400 $\left.\mathrm{MHz}, \mathrm{CDCl}_{3}\right),{ }^{13} \mathrm{C} \mathrm{NMR}\left(100 \mathrm{MHz}, \mathrm{CDCl}_{3}\right)$.

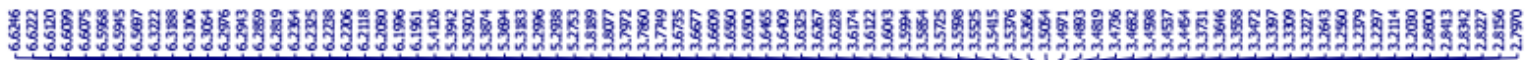<smiles>O=C(C(CBr)C1C=CC=CC=C1)N1CCOCC1</smiles>
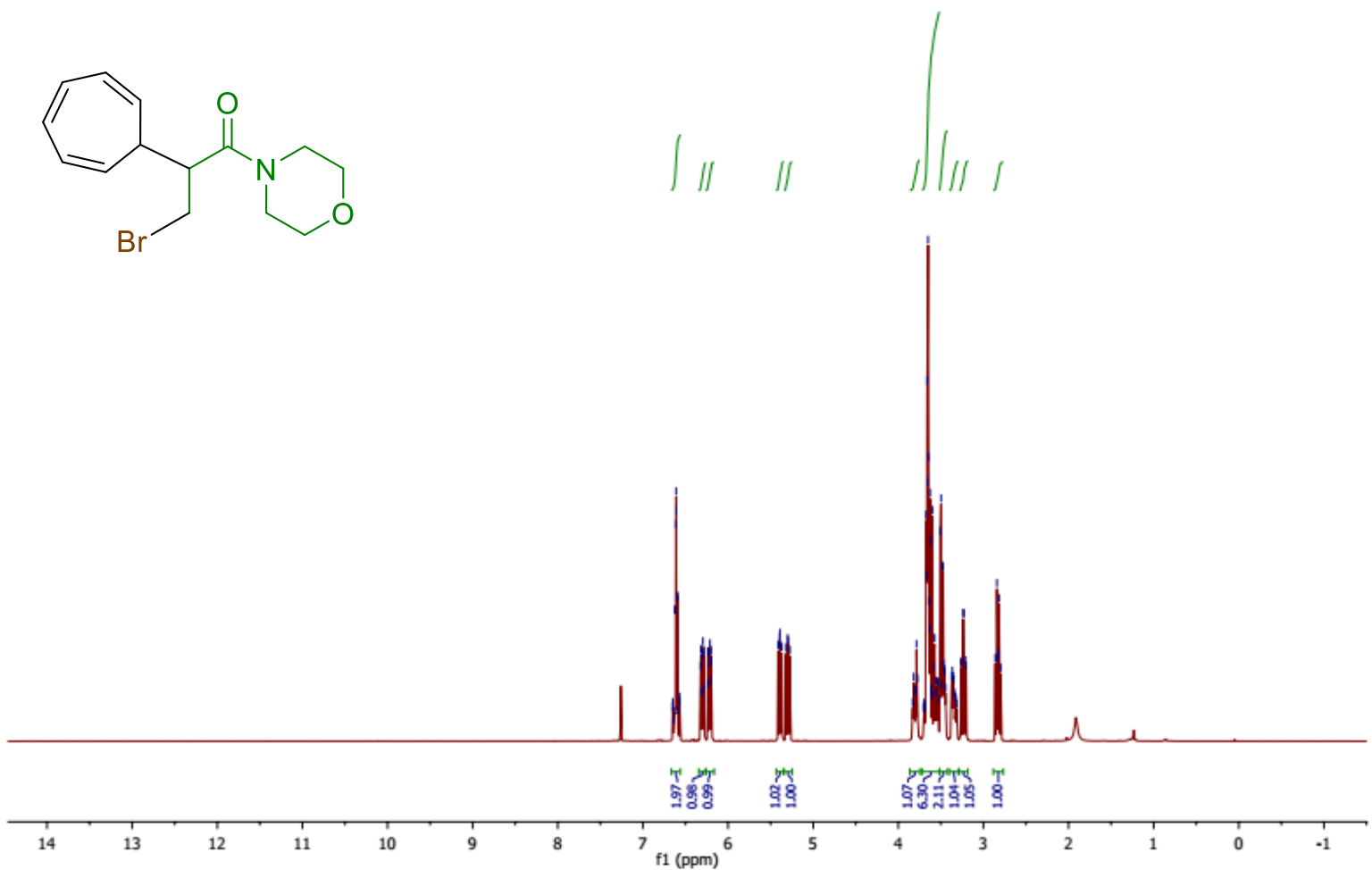

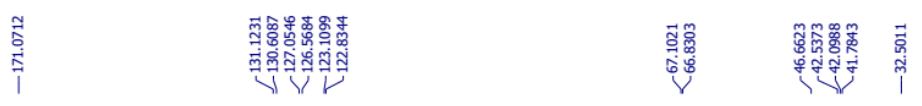

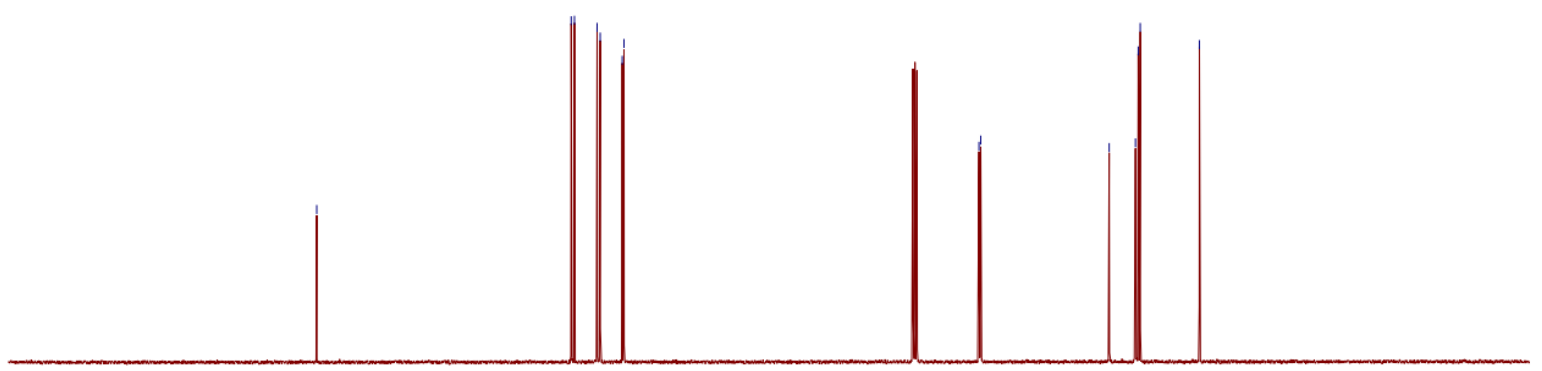

$\begin{array}{lllllllllllllllllllllllll}210 & 200 & 190 & 180 & 170 & 160 & 150 & 140 & 130 & 120 & 110 & 100 & 100 & 80 & 70 & 60 & 50 & 40 & 30 & 20 & 10 & 0 & -10\end{array}$ 
3-Bromo-4-(cyclohepta-2,4,6-trien-1-yl)-1-methylpyrrolidine-2,5-dione(4n); ${ }^{1} \mathbf{H} \quad$ NMR (400 $\left.\mathrm{MHz}, \mathrm{CDCl}_{3}\right),{ }^{13} \mathrm{C} \mathrm{NMR}\left(100 \mathrm{MHz}, \mathrm{CDCl}_{3}\right)$.

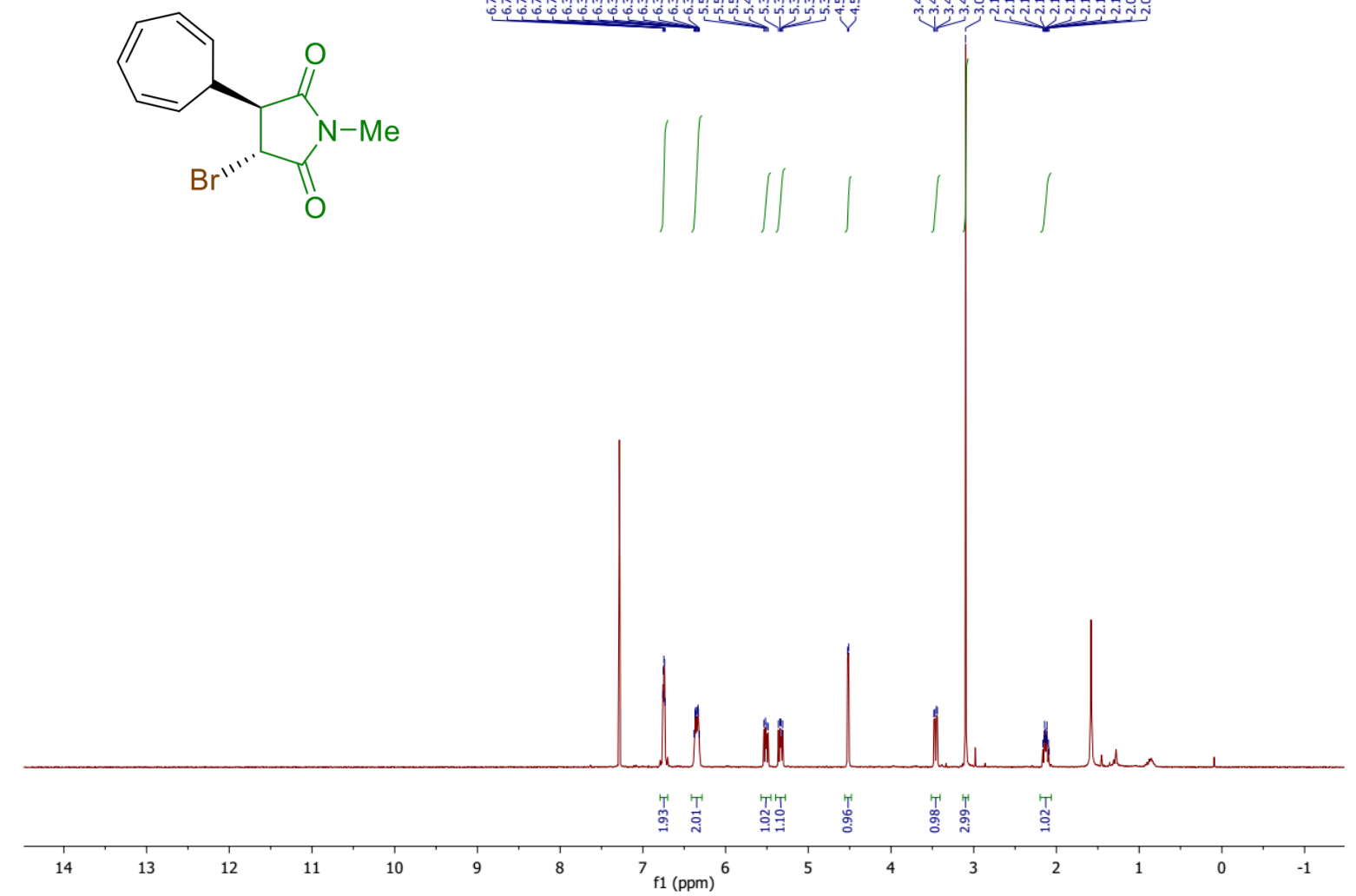

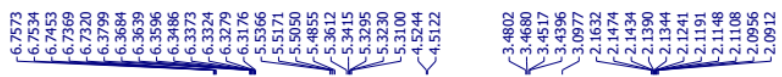

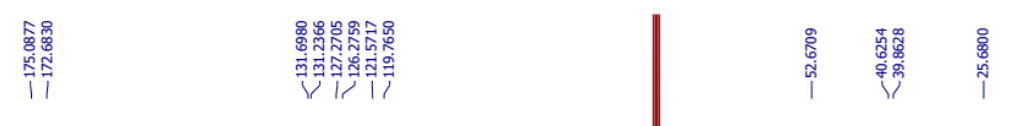

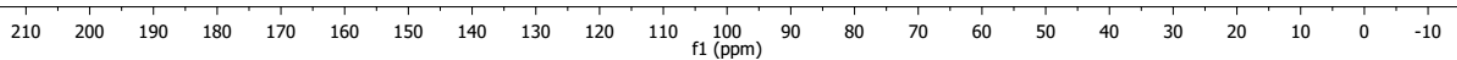


4-Bromo-3-(cyclohepta-2,4,6-trien-1-yl)-5-(cyclohepta-2,4,6-trien-1-yloxy)-3,4-dihydro-2Hpyrrol-2-one (4o); ${ }^{1} \mathrm{H}$ NMR (400 $\left.\mathrm{MHz} \mathrm{CDCl}_{3}\right),{ }^{13} \mathrm{C}$ NMR (100 $\mathrm{MHz}, \mathrm{CDCl}_{3}$ ).

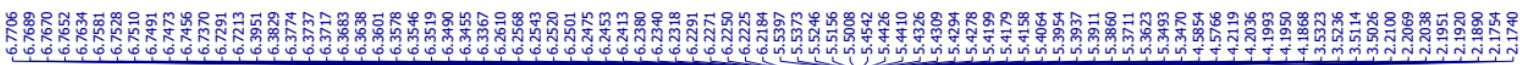<smiles></smiles>
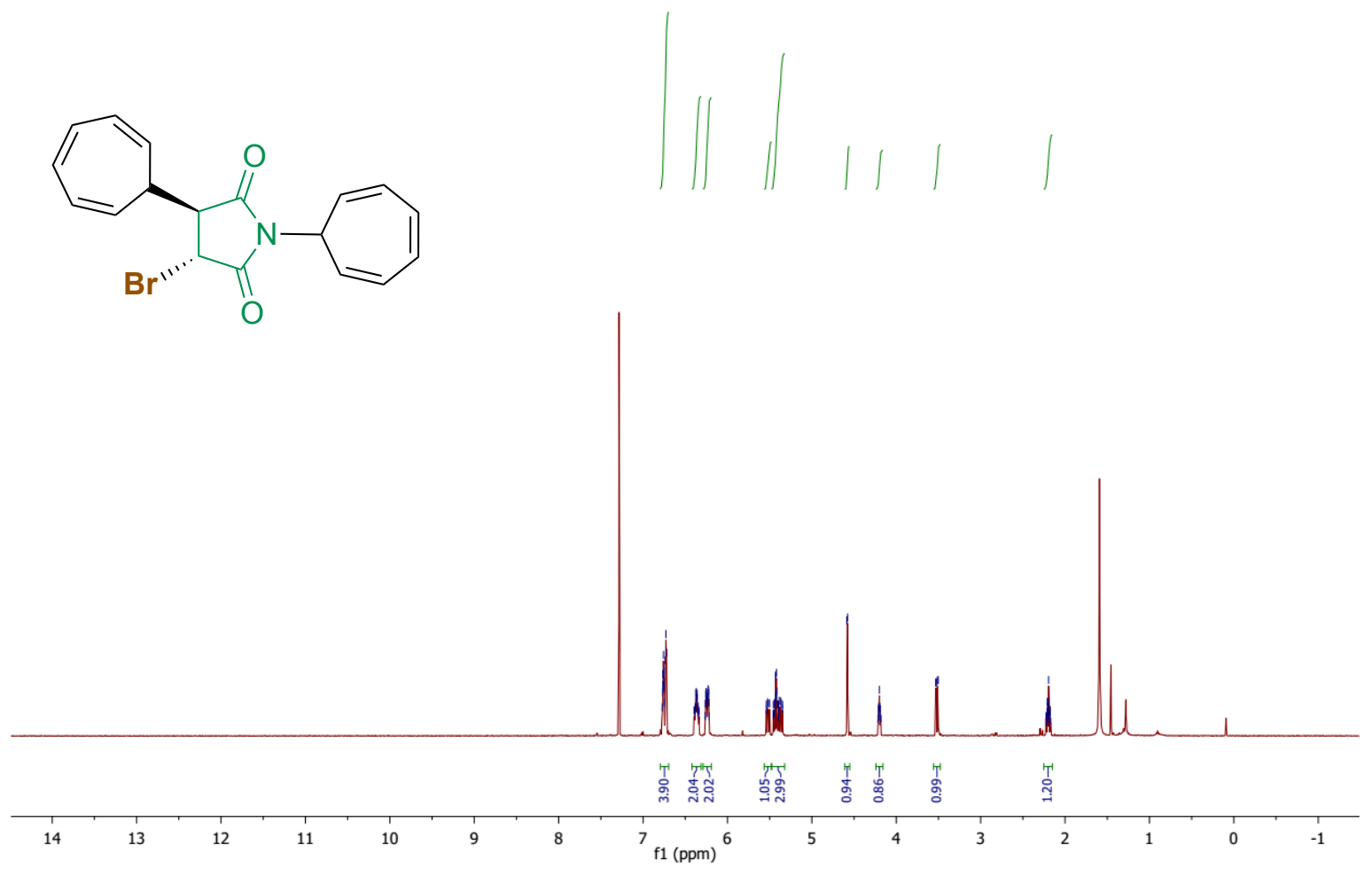

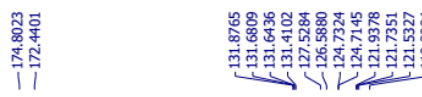

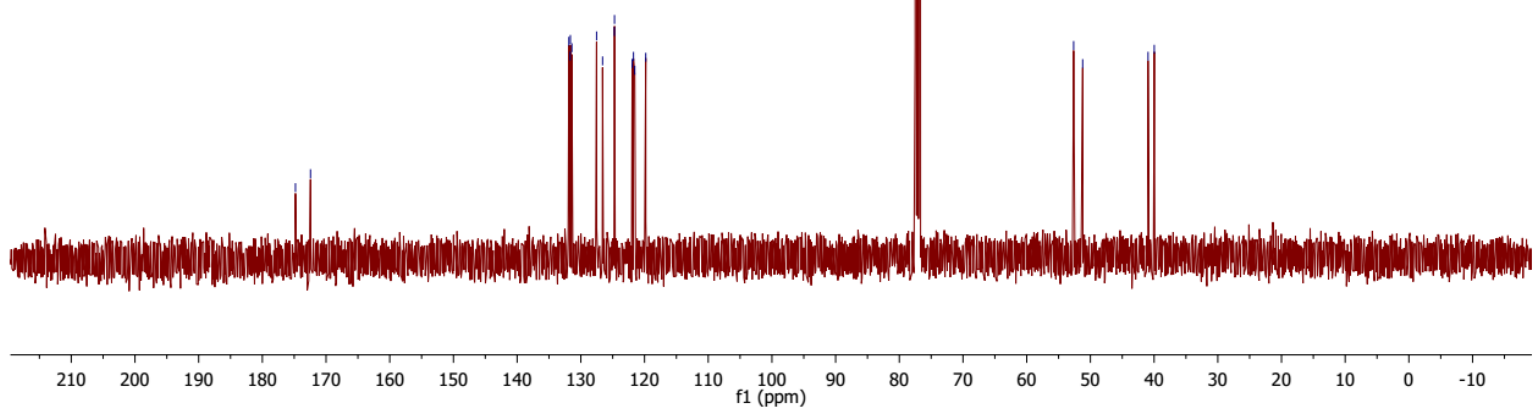


1-Bromo-2-(cyclohepta-2,4,6-trien-1-yl)pentan-3-one (4p); ${ }^{1}$ H NMR (400 MHz, $\left.\mathbf{C D C l}_{3}\right)$, ${ }^{13} \mathrm{C}$ NMR (100 MHz, $\left.\mathrm{CDCl}_{3}\right)$.

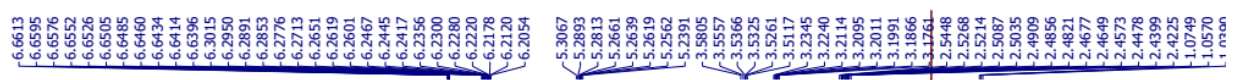<smiles>CCC(=O)C(CBr)c1ccccc1</smiles>

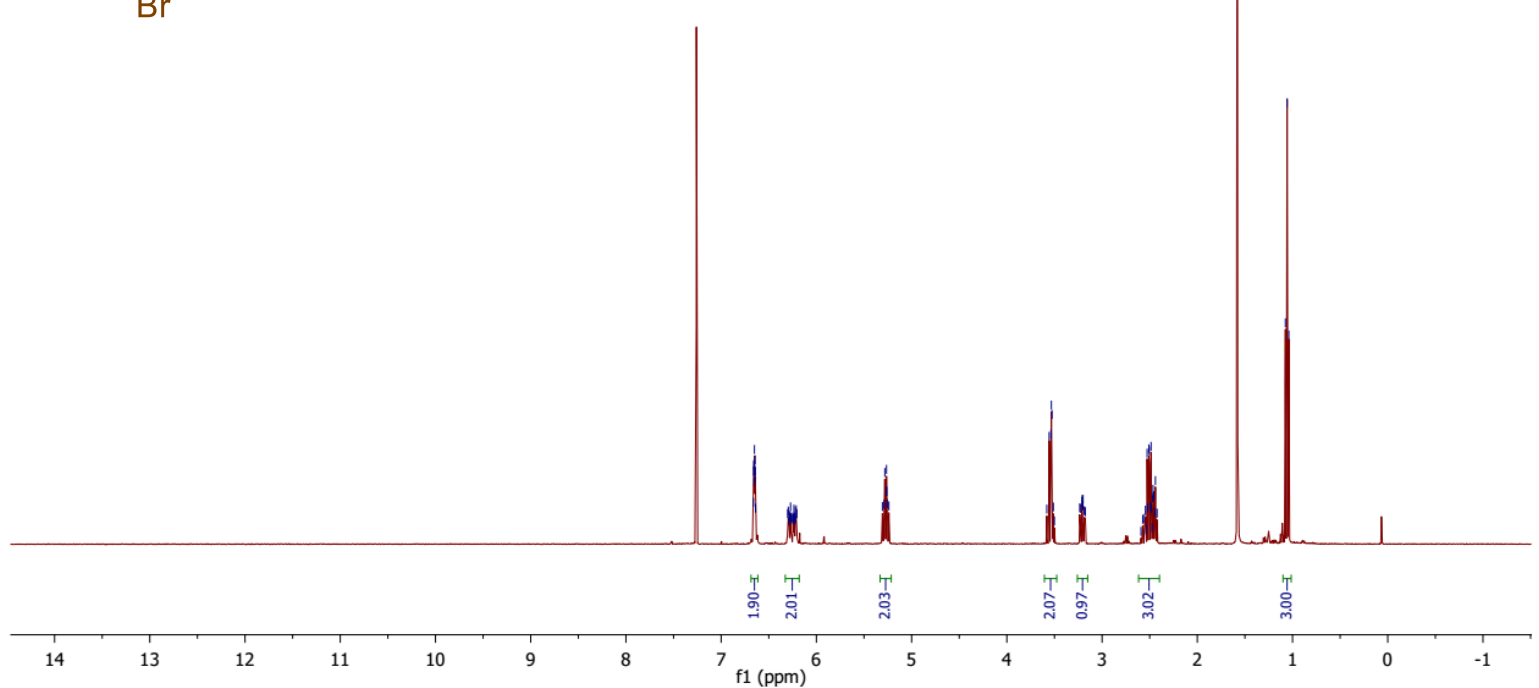

商 
5-(cyclohepta-2,4,6-trien-1-yl)furan-2(5H)-one (4q); ${ }^{1} \mathbf{H}$ NMR (400 $\left.\mathbf{M H z}, \mathbf{C D C l}_{3}\right),{ }^{13} \mathbf{C}$ NMR (100 MHz, CDCl $)_{3}$.

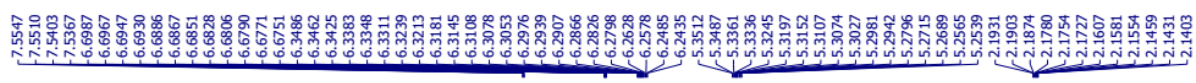
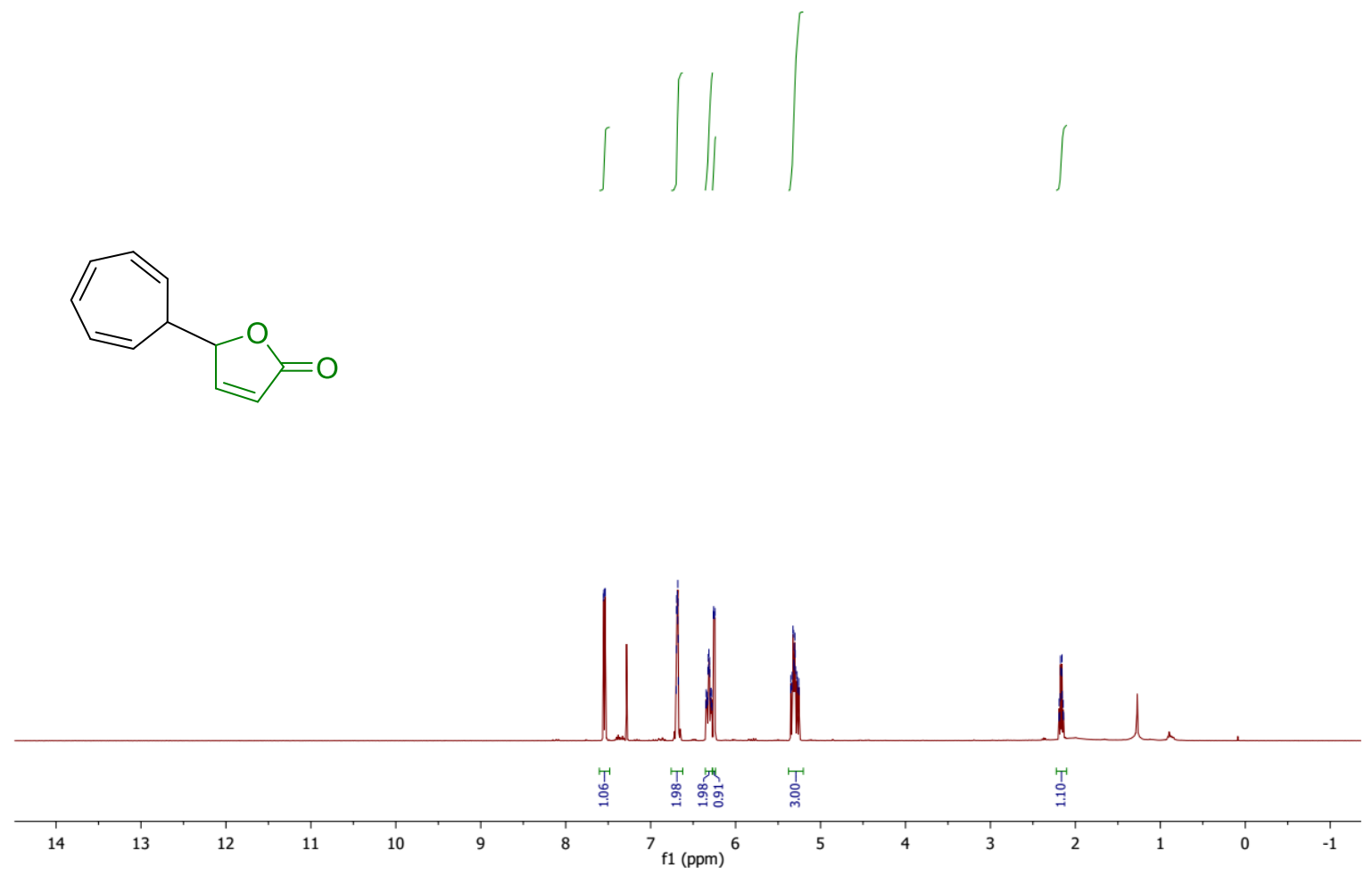

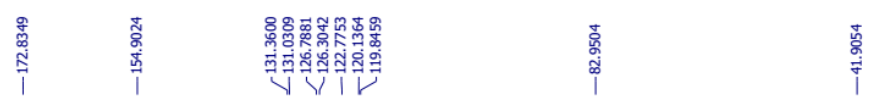

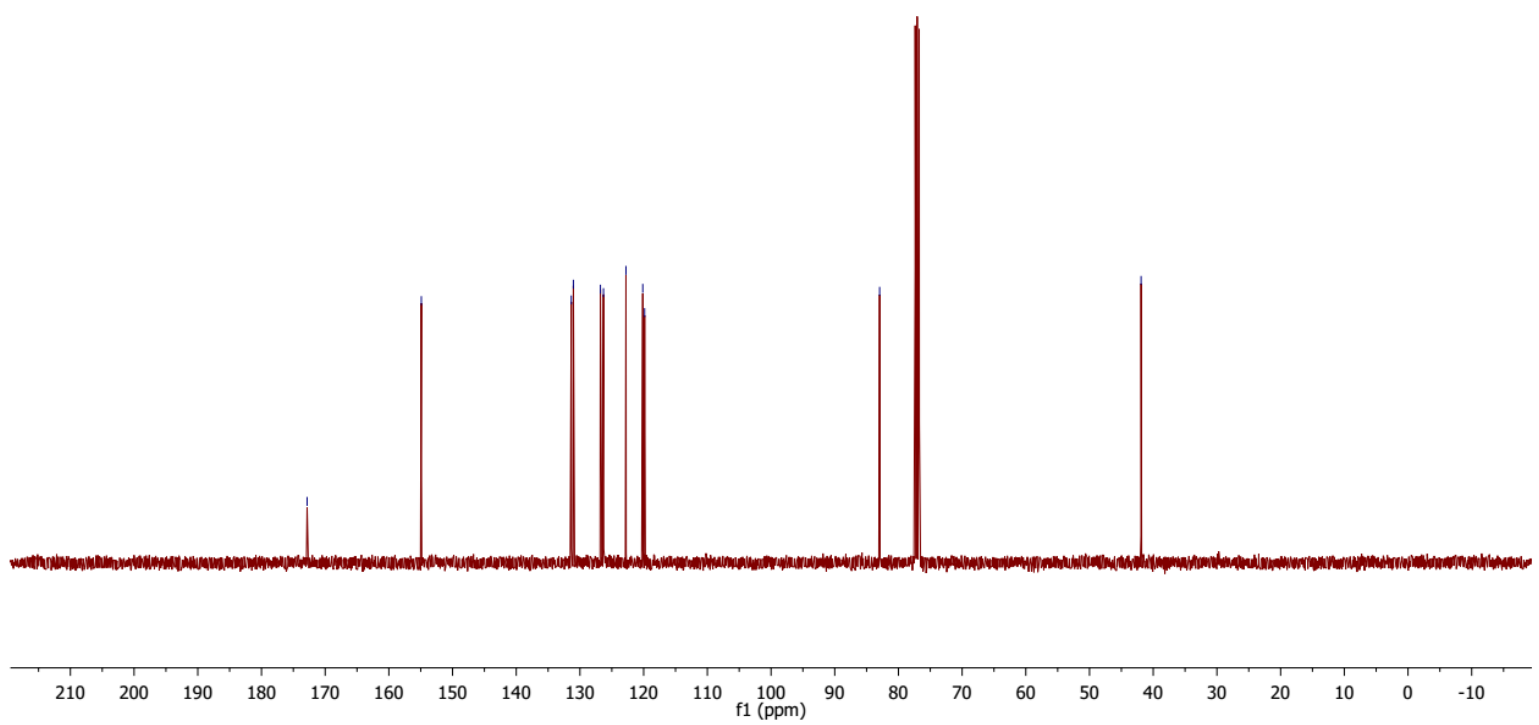


4-oxo-5-phenylpentanoic acid (4q'): ${ }^{1} \mathrm{H}$ NMR (400 MHz, $\left.\mathrm{CDCl}_{3}\right),{ }^{13} \mathrm{C}$ NMR (100 MHz, $\left.\mathrm{CDCl}_{3}\right)$.
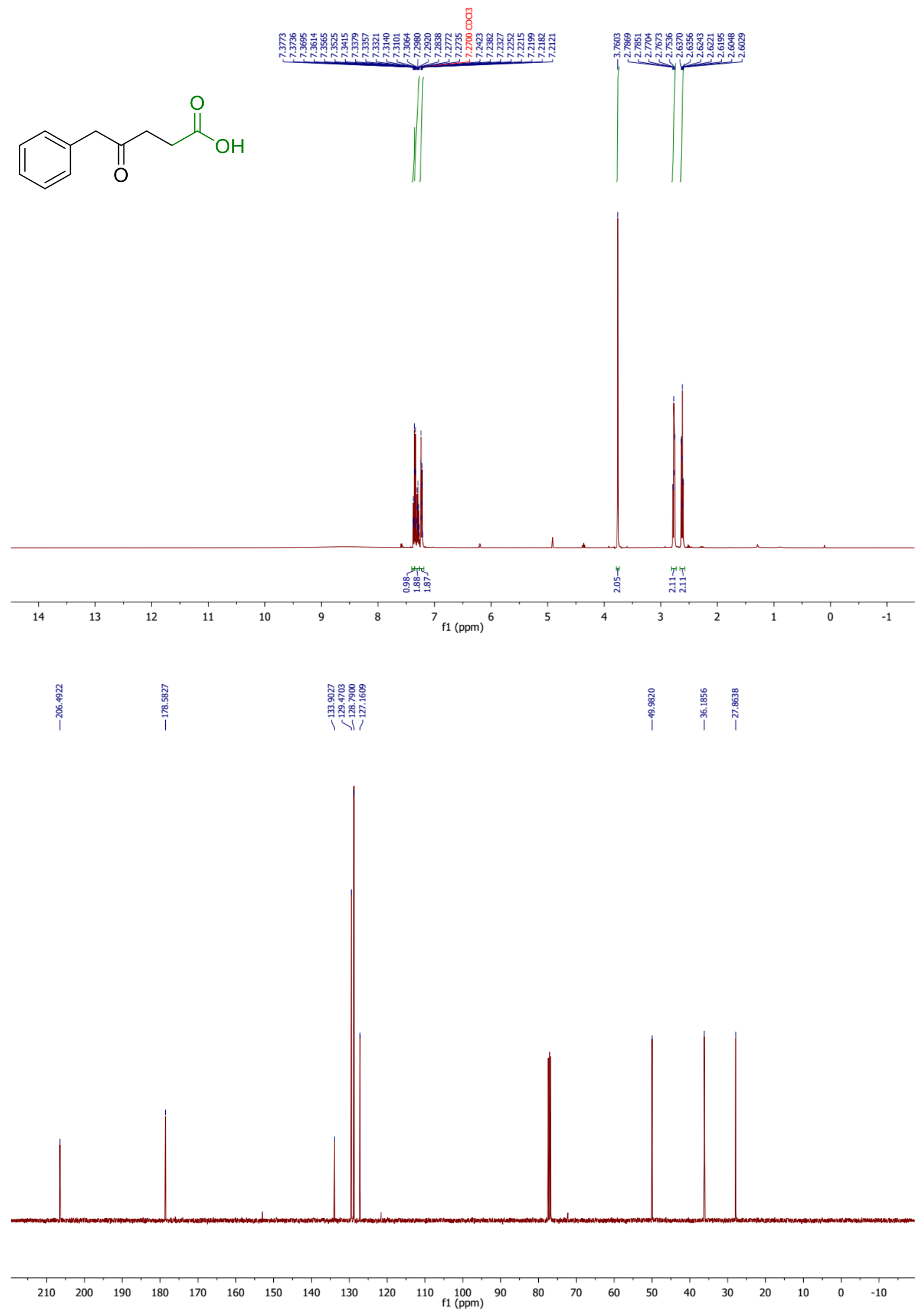

S51 
2-Hydroxyethyl 3-bromopropanoate (4r); ${ }^{1} \mathrm{H}$ NMR (400 $\left.\mathbf{M H z}, \mathrm{CDCl}_{3}\right),{ }^{13} \mathrm{C}$ NMR (100 $\left.\mathrm{MHz}, \mathrm{CDCl}_{3}\right)$.<smiles>O=C(CBr)OCCO</smiles><smiles>C1#CC2C=CC12</smiles>
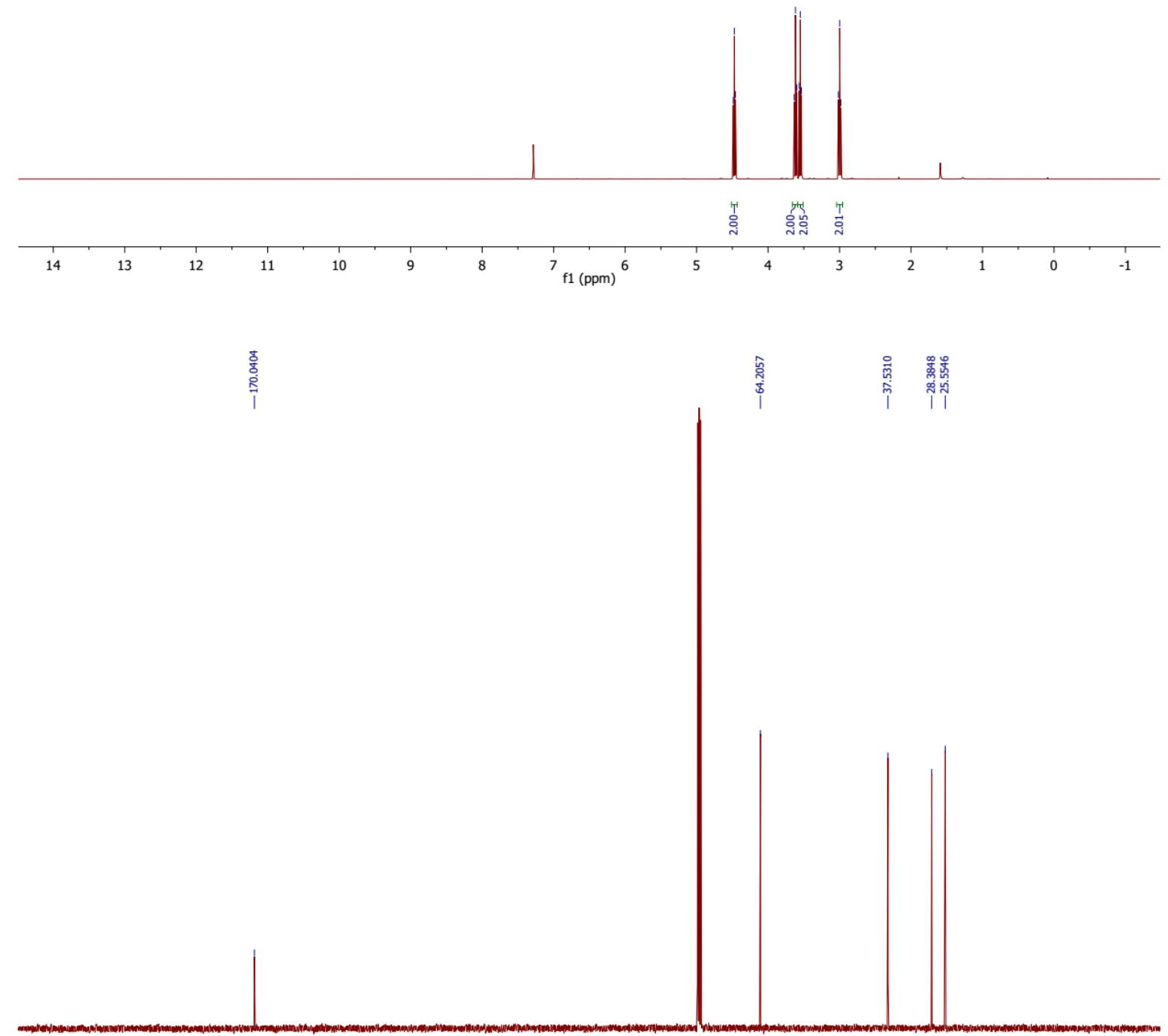

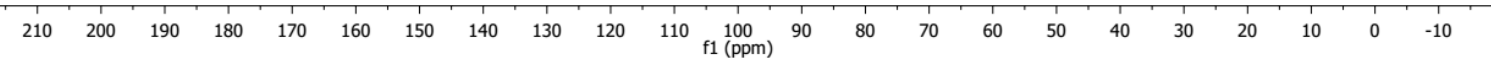


Methyl (E)-3-bromo-2-(cyclohepta-2,4,6-trien-1-yl)acrylate (6a: $\mathbf{Z}$ and $\mathbf{E}$ mixture $\sim \mathbf{9}: \mathbf{1}$ ratio); ${ }^{1} \mathrm{H}$ NMR (400 MHz, $\left.\mathrm{CDCl}_{3}\right),{ }^{13} \mathrm{C} \mathrm{NMR}\left(100 \mathrm{MHz}, \mathrm{CDCl}_{3}\right)$.
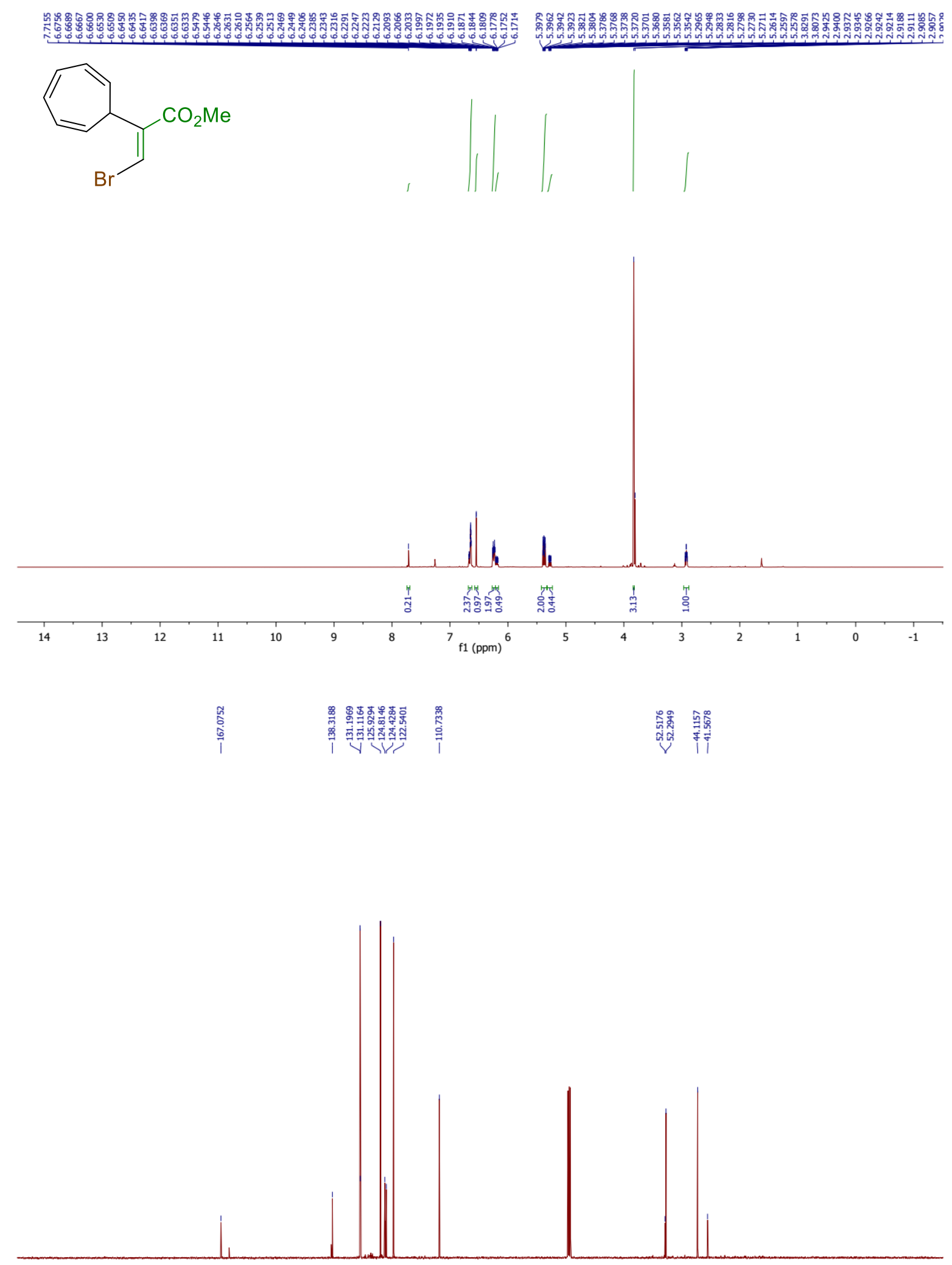

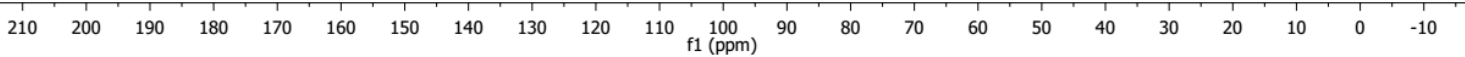


Ethyl (Z)-3-bromo-2-(cyclohepta-2,4,6-trien-1-yl)acrylate (6b); ${ }^{1} \mathbf{H}$ NMR (400 MHz, $\left.\mathrm{CDCl}_{3}\right),{ }^{13} \mathrm{C}$ NMR (100 MHz, $\left.\mathrm{CDCl}_{3}\right)$.

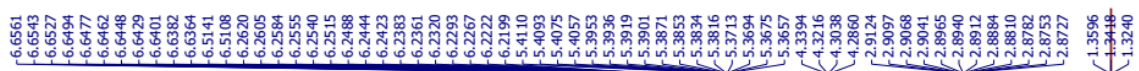<smiles>CCOC(=O)/C(=C\Br)C1C=CC=CC=C1</smiles>

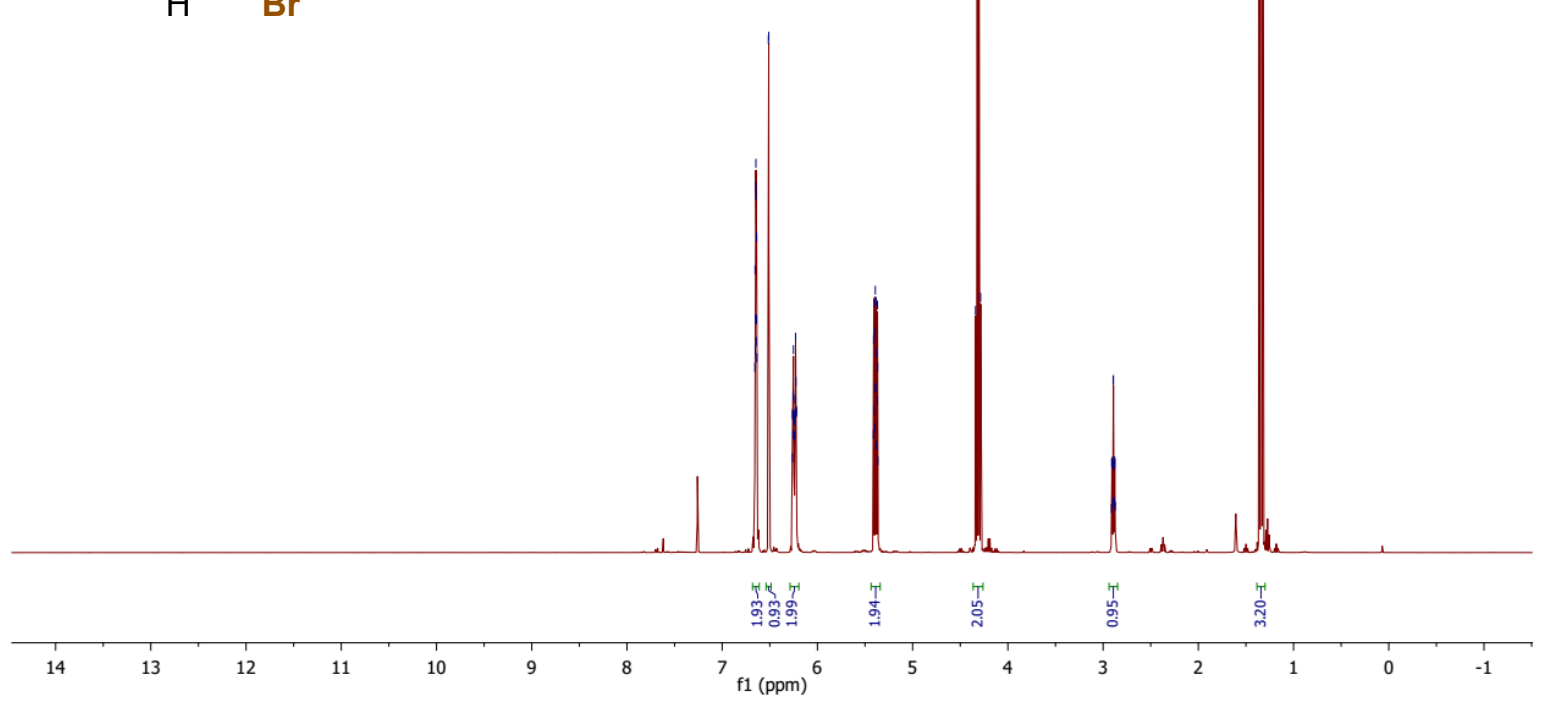

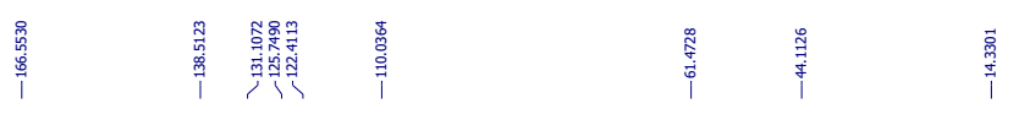

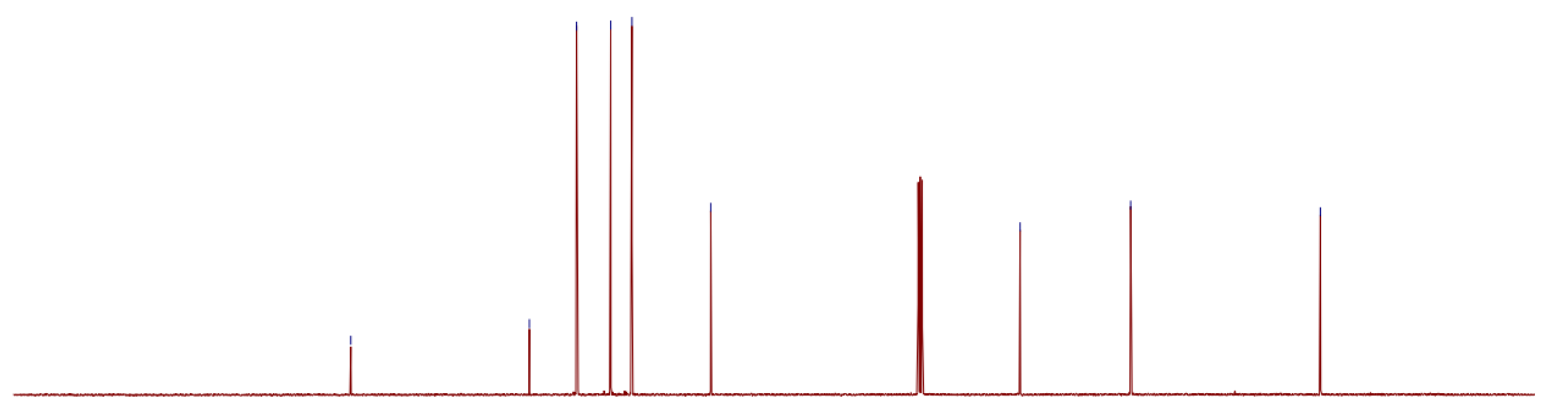

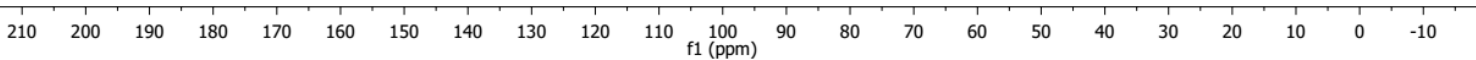


Dimethyl 2-bromo-3-(cyclohepta-2,4,6-trien-1-yl)but-2-enedioate (6c) two isomers; ${ }^{1} \mathbf{H}$ NMR (400 MHz, $\left.\mathrm{CDCl}_{3}\right),{ }^{13} \mathrm{C}$ NMR (100 MHz, $\left.\mathrm{CDCl}_{3}\right)$.<smiles>CCOC(OC)C(Br)=C(C(C)=O)C1C=CC=CC=C1</smiles>
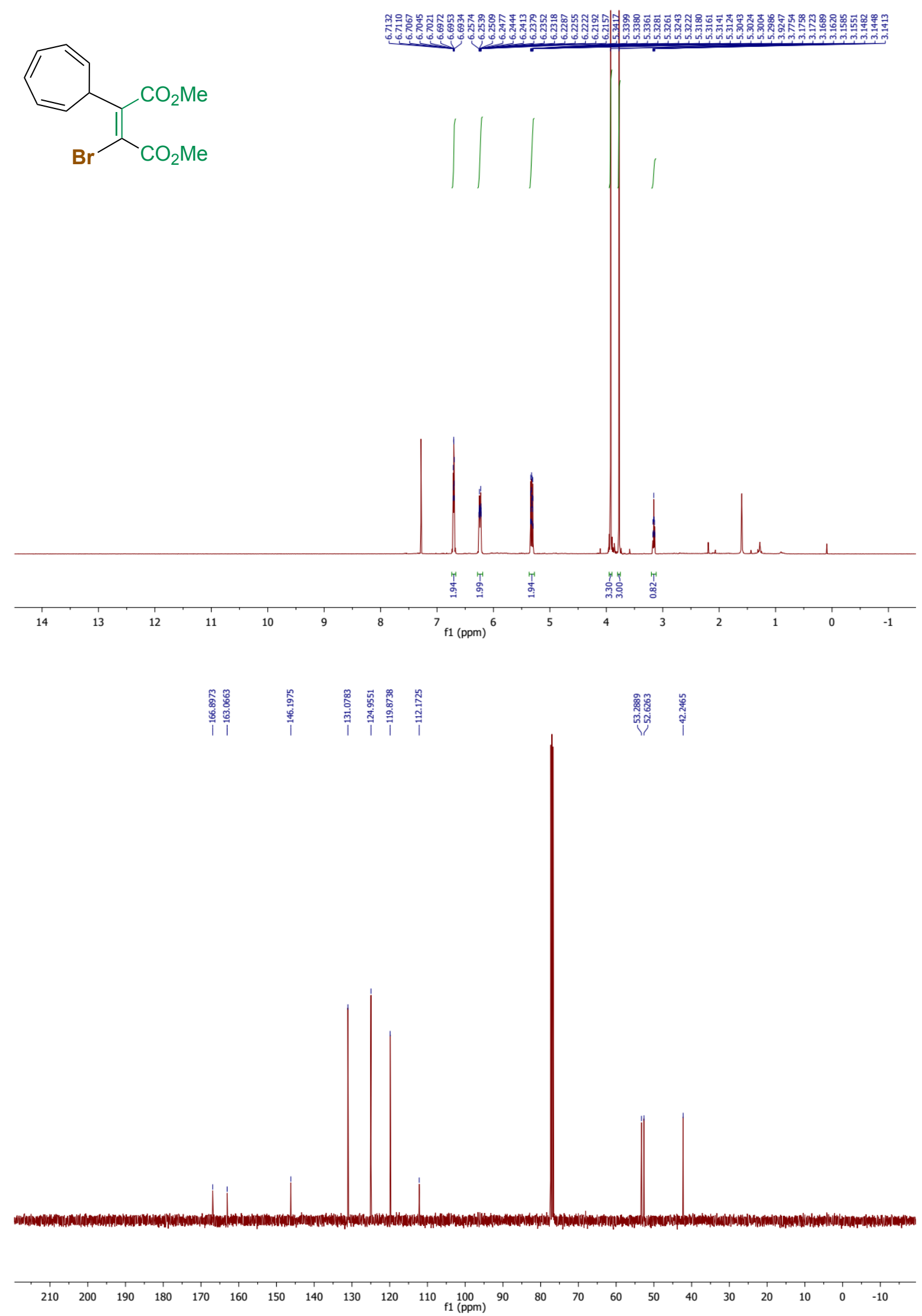


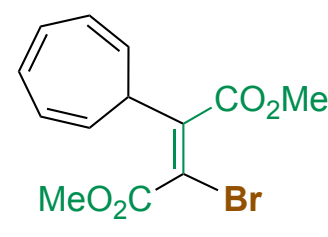

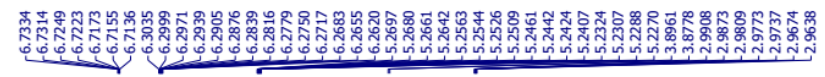

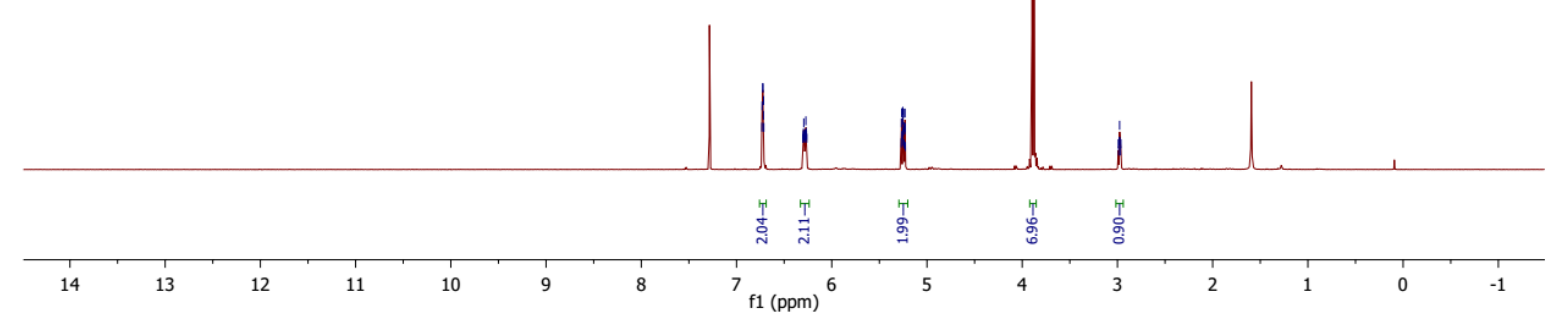

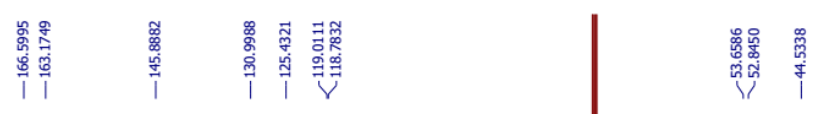

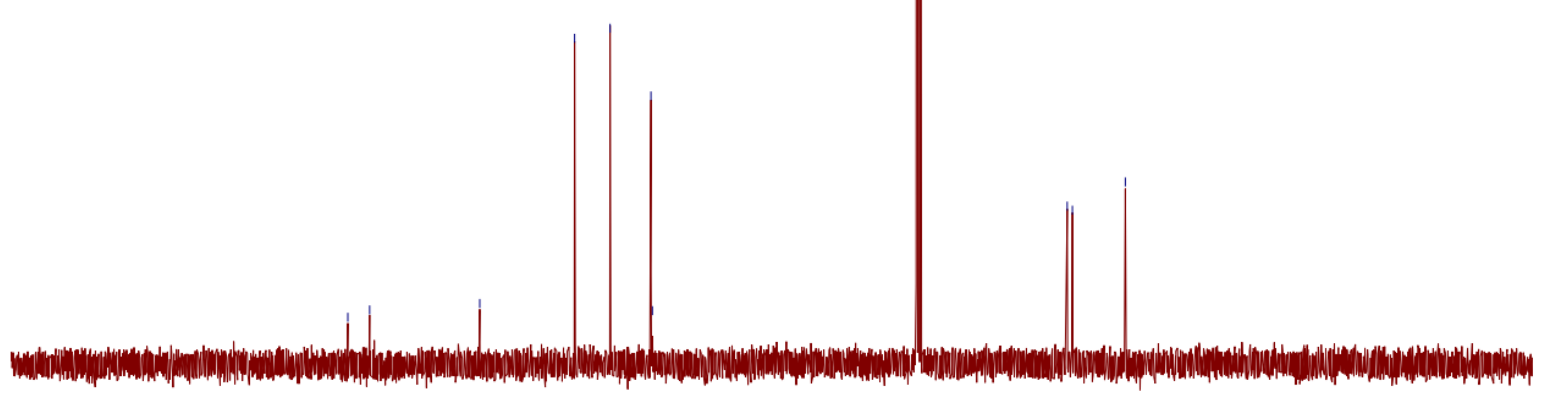

$\begin{array}{lllllllllllllllllllllll}210 & 200 & 190 & 180 & 170 & 160 & 150 & 140 & 130 & 120 & 110 & 100 & 90 & 80 & 70 & 60 & 50 & 40 & 30 & 20 & 10 & 0 & -10\end{array}$ 
Methyl 2-(cyclohepta-2,4,6-trien-1-yl)acrylate (12); ${ }^{\mathbf{1}} \mathbf{H} \mathbf{N M R}\left(400 \mathrm{MHz}, \mathrm{CDCl}_{3}\right),{ }^{\mathbf{1 3}} \mathbf{C} \mathbf{~ N M R}$ $\left(100 \mathrm{MHz}, \mathrm{CDCl}_{3}\right)$.
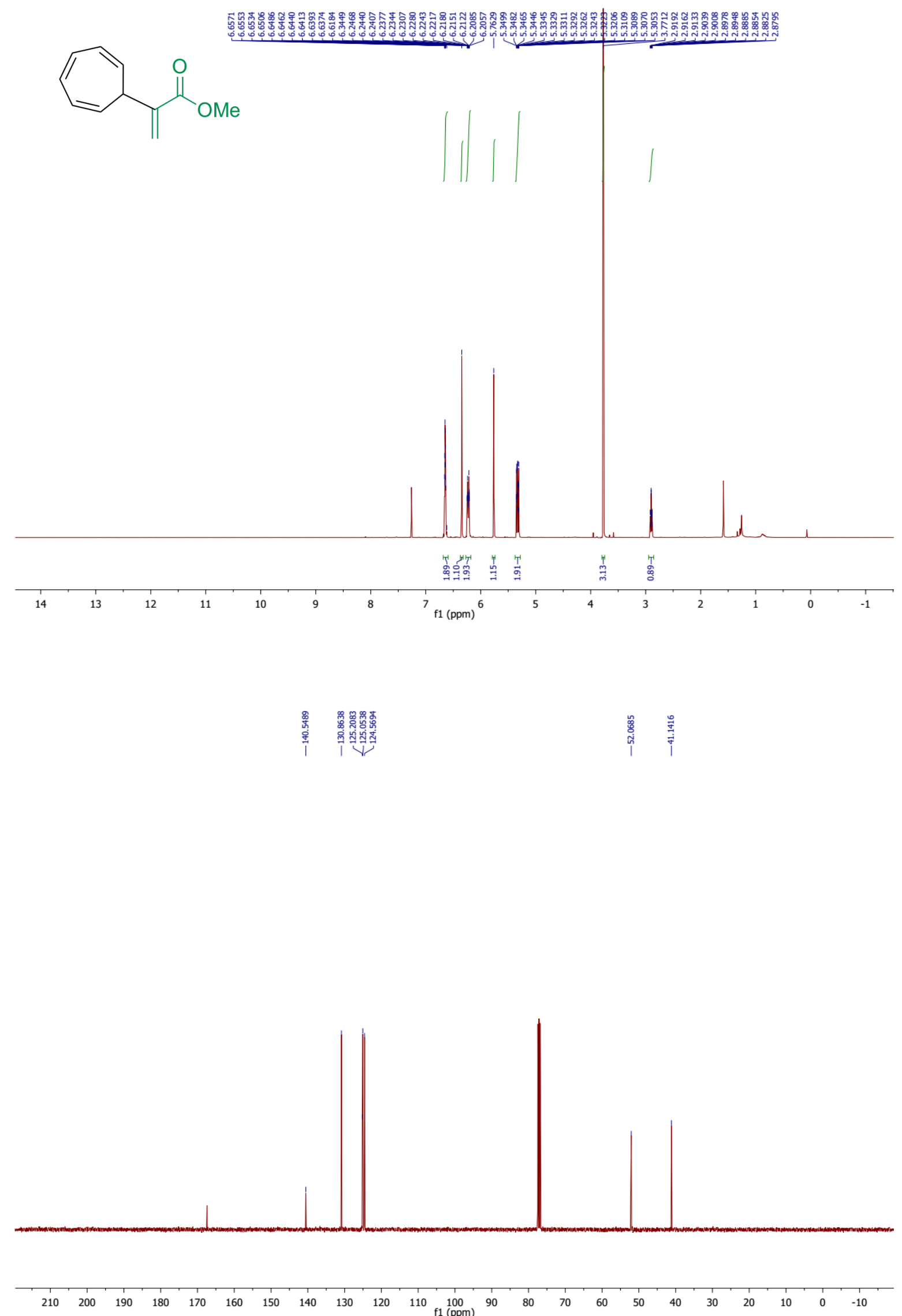
Methyl 3-bromo-2-(1,3-dioxodecahydro-4,6-ethanocyclopropa[f]isoindol-5-yl) propanoate (13); ${ }^{1} \mathbf{H}$ NMR $\left(400 \mathrm{MHz}, \mathrm{CDCl}_{3}\right),{ }^{13} \mathbf{C}$ NMR $\left(100 \mathrm{MHz}, \mathrm{CDCl}_{3}\right)$.
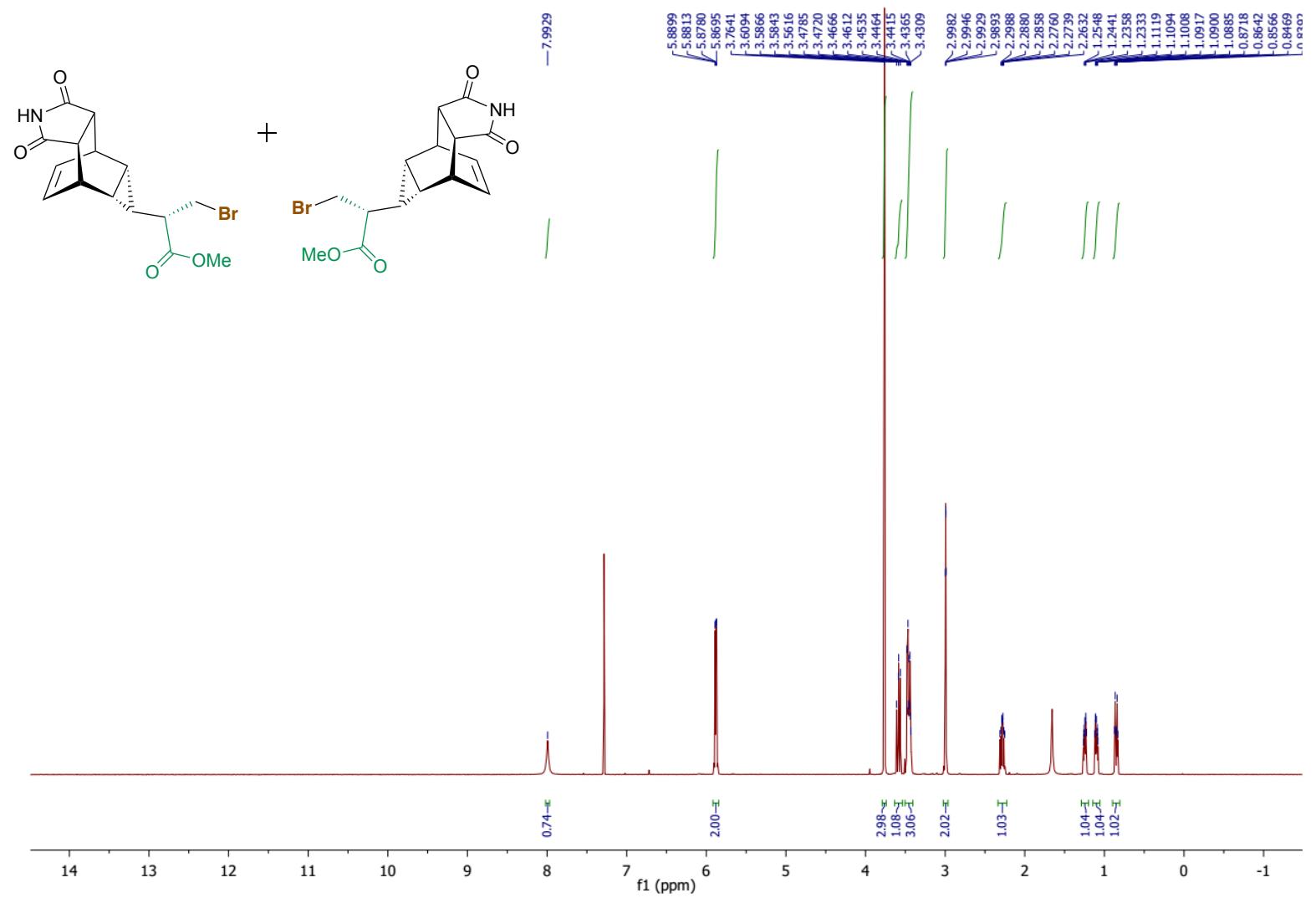

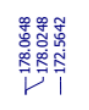

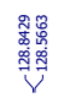

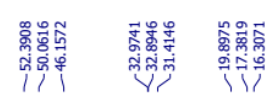
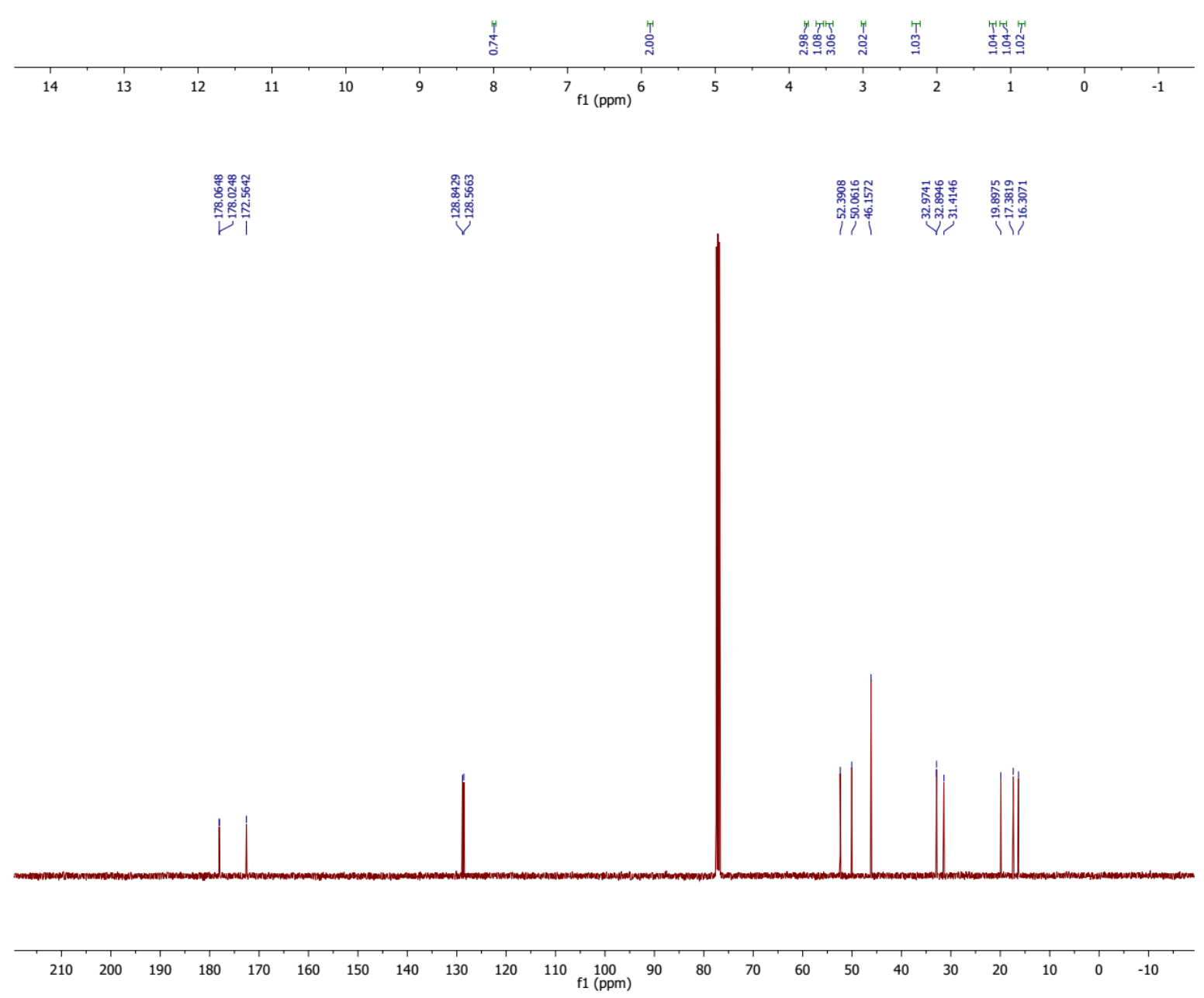

S58 
Methyl 2-bromo-2-phenyl-3-hydroxypropanoate (14); ${ }^{1} \mathrm{H}$ NMR (400 $\left.\mathbf{M H z}, \mathrm{CDCl}_{3}\right),{ }^{13} \mathrm{C}$ NMR (100 MHz, $\left.\mathrm{CDCl}_{3}\right)$.
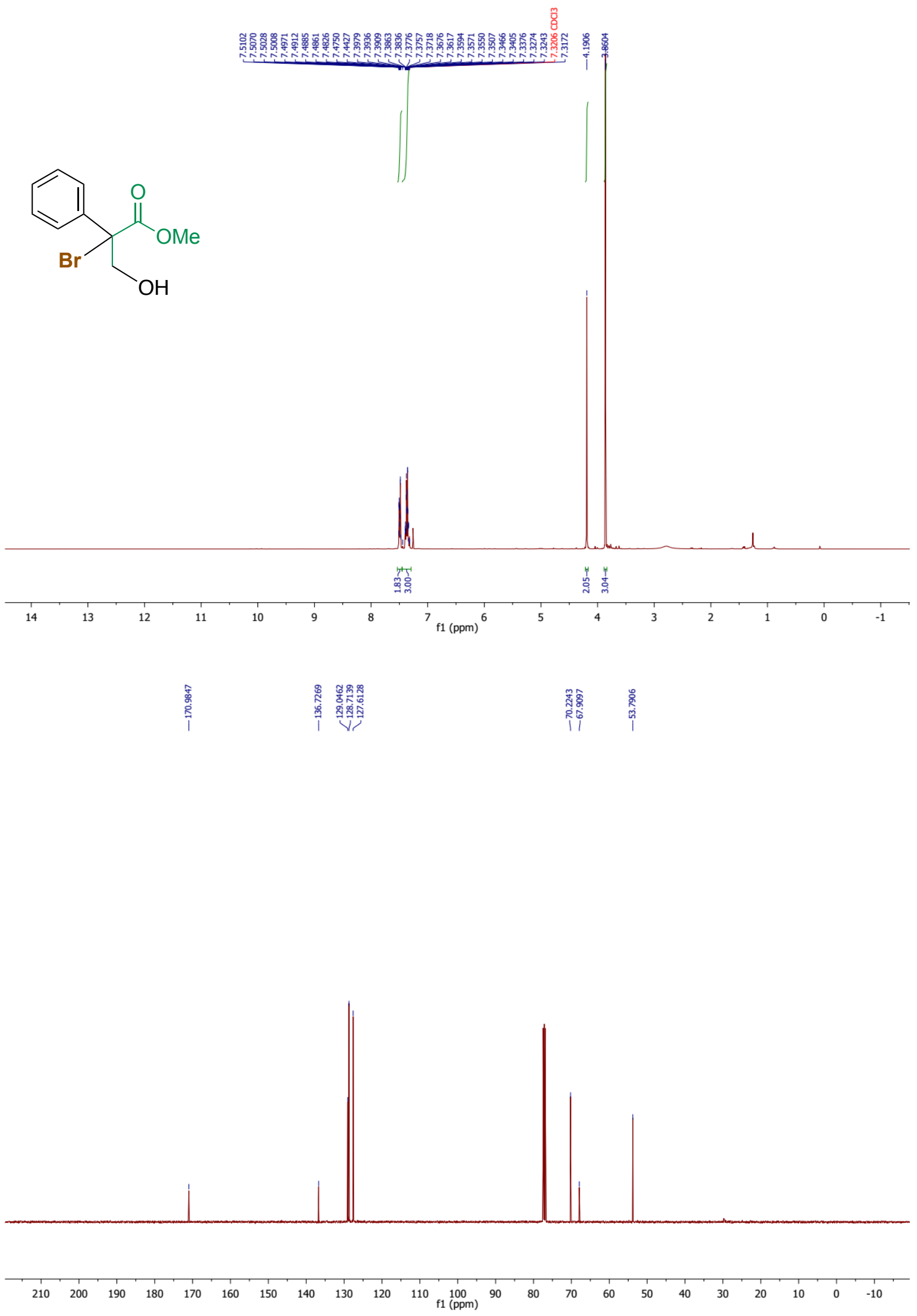

S59 


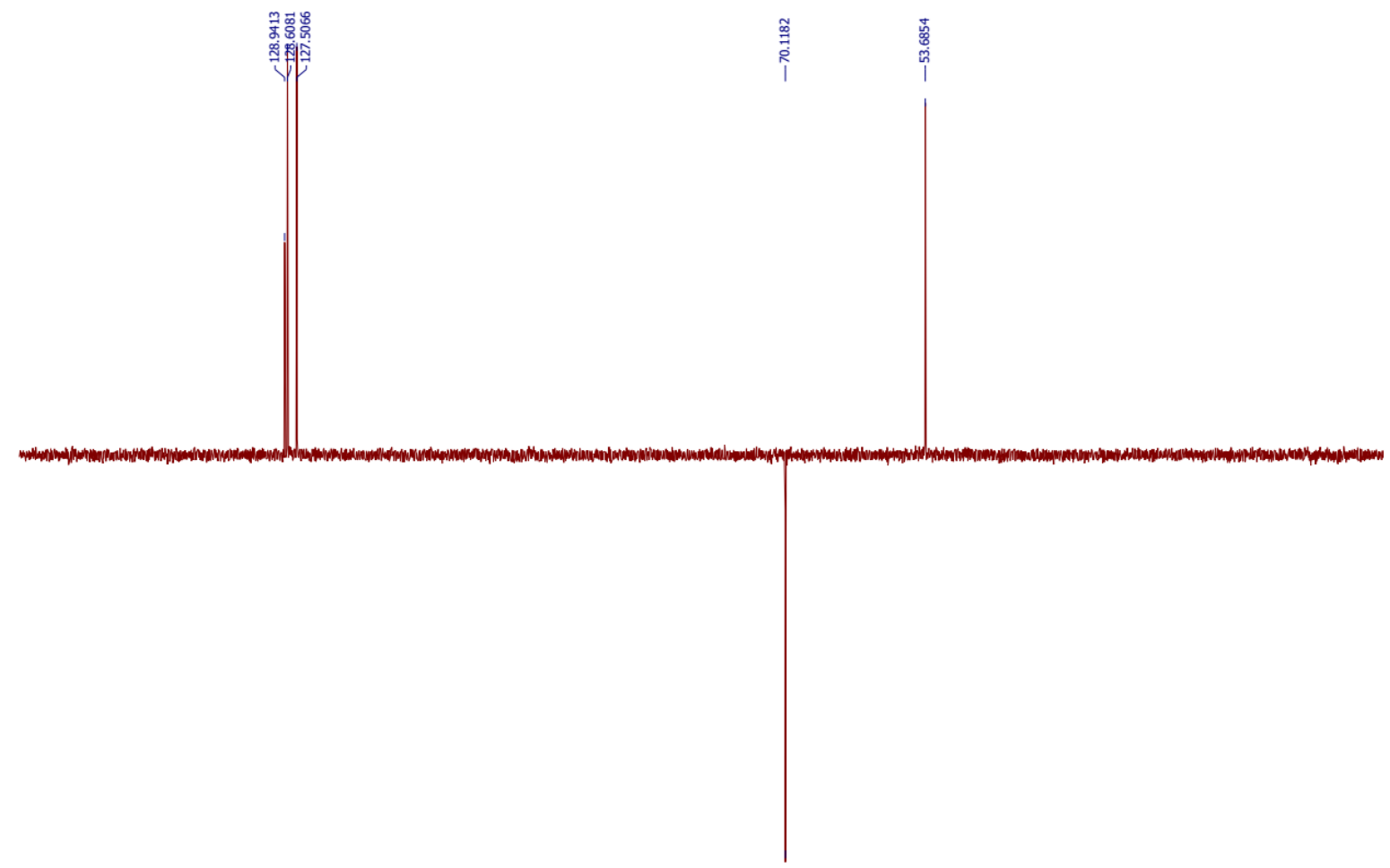

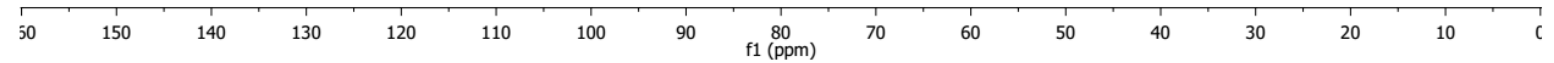

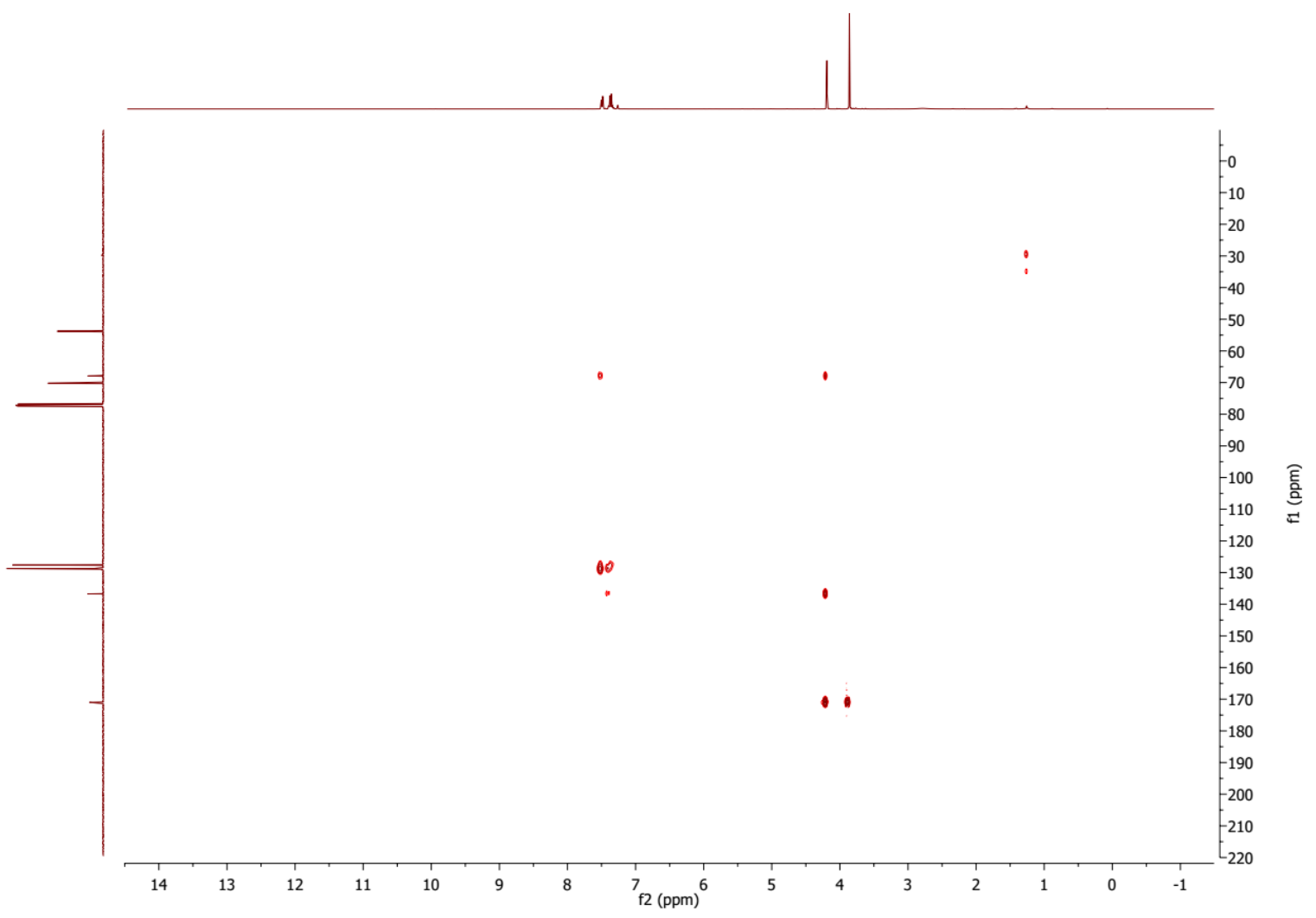


Methyl 2-(bromomethyl)-3-chloro-3-phenyl-3-propanoate

(15, mixture

diastereoisomers); ${ }^{1} \mathbf{H}$ NMR $\left(300 \mathrm{MHz}, \mathrm{CDCl}_{3}\right),{ }^{13} \mathbf{C} \mathbf{N M R}\left(75 \mathrm{MHz}, \mathrm{CDCl}_{3}\right)$.

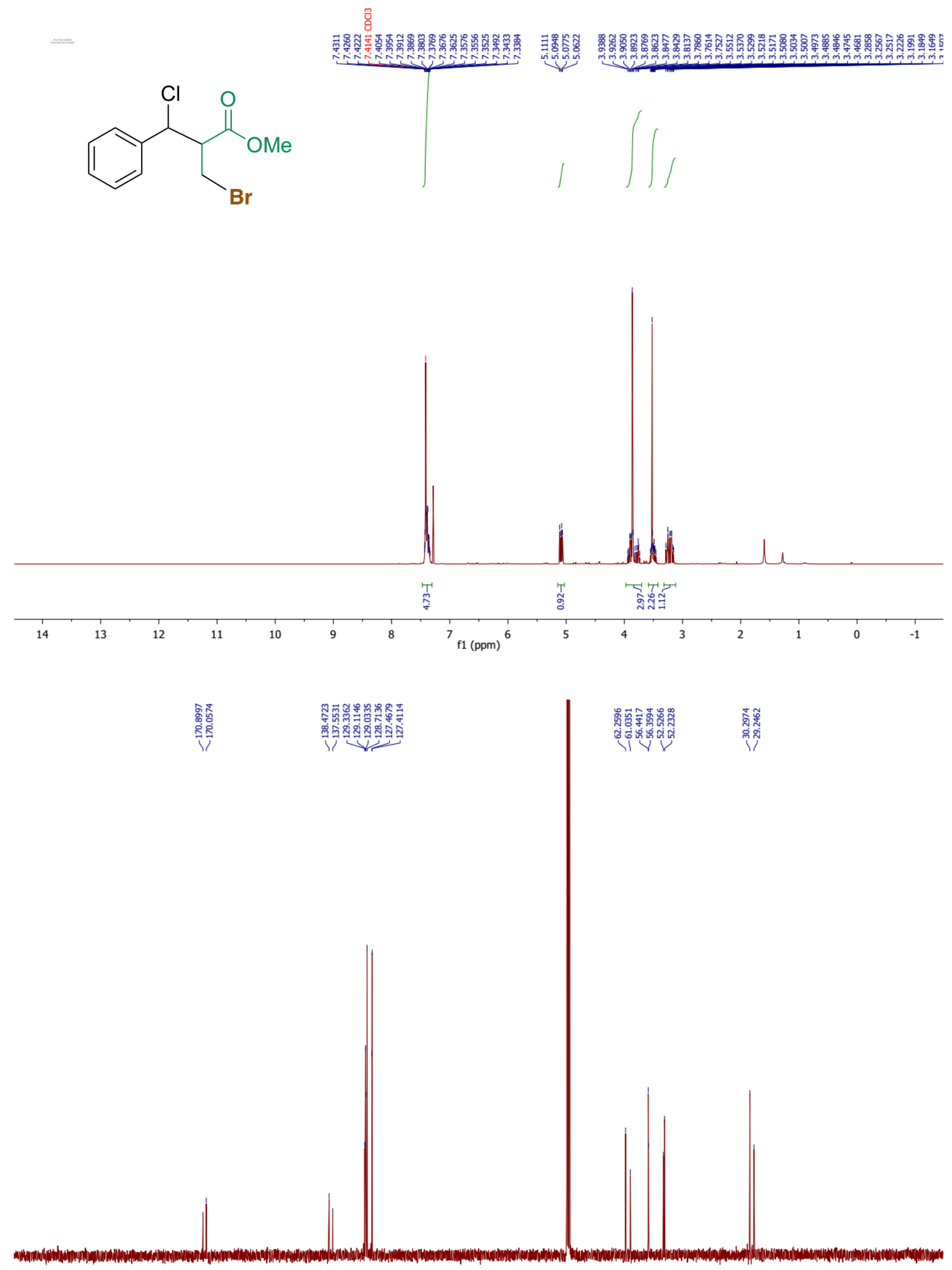

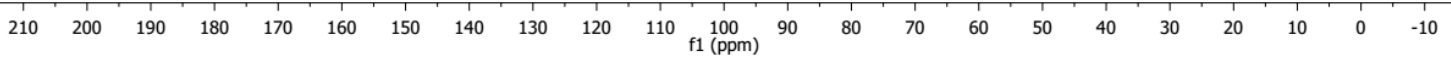


